\begin{abstract}
SHIN, GWANSEOB. Viscoelastic Responses of the Lumbar Spine During Prolonged Stooping. (Under the direction of Dr. Gary A. Mirka and Dr. Elizabeth G. Loboa)
\end{abstract}

There is considerable evidence that awkward postures of the low back are related to the incidence of low back disorders (LBDs). Specifically, the stooped or fully flexed posture maintained over a prolonged period of time has been known to lead to LBDs in many industrials tasks but the specific biomechanics/physiology of this link is not fully developed. This study combined empirical work with finite element analyses to explore this relationship.

The empirical work focused on quantifying the time-dependent responses of the lumbar spine during a prolonged stooped posture by assessing the changes in the sagittal plane range of lumbar flexion and the electromyographic activity of the back extensor musculature in the isokinetic lifts during and after prolonged stooping. Ten healthy participants performed a regimen of a 10-minute stooping period followed by a 10-minute upright standing recovery period, with an isokinetic lift every 2.5 minutes. Results showed significant creep effects of the flexion angle and the increased activity of extensor muscles during stooping to compensate for the reduced extensor moment producing capability of the passive tissues. The 10-minute upright standing did not produce a full recovery of the lumbar spine tissues but a 30 -second rest break in the middle of the stooping period moderated these viscoelastic responses.

A three-dimensional finite element (FE) model of the lumbar spine was developed to predict the responses of the passive and active tissues of the low back during the prolonged stooping and recovery period. This model employed a nonlinear stress-strain relationship 
describing the viscoelastic material properties of individual components of the lumbar spine. The trunk flexion tasks that were performed in the in vivo empirical work were simulated in the FE model and the predicted results (range of motion, muscle activation levels, etc.) were compared with experimental results to validate the model. The predicted results by the FE model showed high correlation $(\mathrm{R}>0.9)$ with the in vivo experimental results, confirming the capability of the FE model as a potential tool for risk assessment of the prolonged stooping tasks.

Results of the in vivo experiment suggested the importance of proper duty cycles in reducing LBD risks due to repetitive prolonged stooping in work-related tasks. The FE model of this study showed potential to simulate various prolonged stooped postures in occupational tasks and predict time-dependent stress/strain of individual spinal tissues. The data from these simulations can be used to design better work postures and duty cycles that can reduce the risks for LBDs, without sacrificing work productivity. 


\title{
VISCOELASTIC RESPONSES OF THE LUMBAR SPINE DURING PROLONGED STOOPING
}

\author{
by \\ GWANSEOB SHIN \\ A dissertation submitted to the Graduate Faculty of \\ North Carolina State University \\ in partial fulfillment of the \\ requirements for the Degree of \\ Doctor of Philosophy
}

INDUSTRIAL ENGINEERING

Raleigh

2005

\section{APPROVED BY}
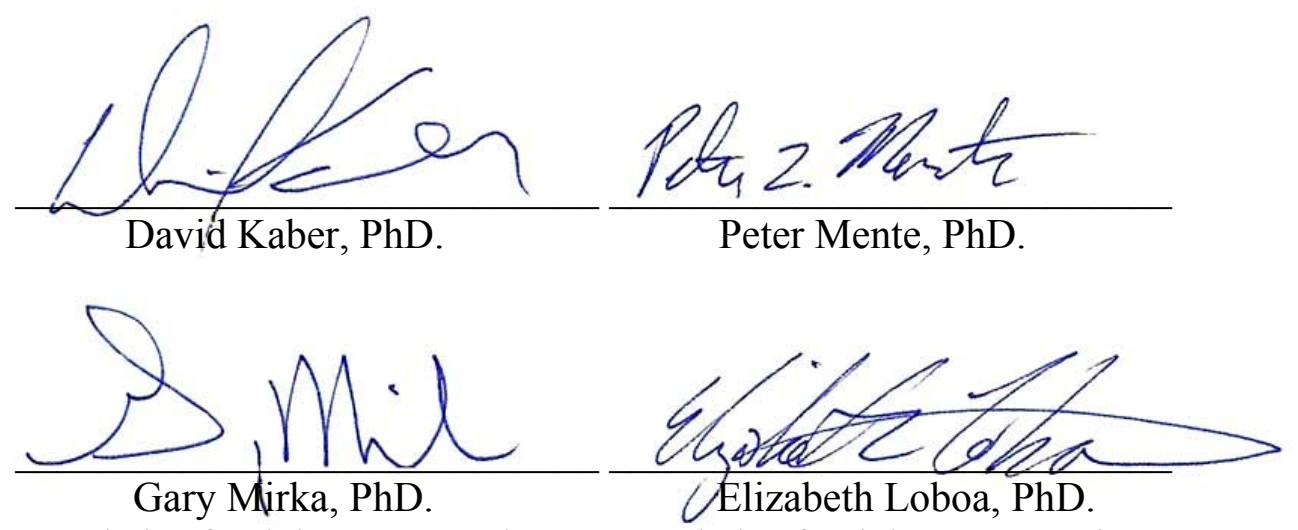

Co-chair of Advisory Committee Co-chair of Advisory Committee 


\section{BIOGRAPHY}

Gwanseob Shin was born in Seoul, Korea in 1973. After graduating from Korea University (Seoul, Korea) with a Bachelors of Engineering in Mechanical Engineering, he was employed at Hanjin Heavy Industries (Pusan, Korea) as a mechanical engineer. In 2000, he joined ergonomics program at North Carolina State University to start his Master's degree under Dr. Gary Mirka in Industrial Engineering. In 2002, he graduated with his Masters of Science in Industrial Engineering and entered the PhD program at NCSU. One year later, he married Jihye Kim after nine years of dating. During his Masters and $\mathrm{PhD}$ programs, Gwanseob was actively involved in many research studies at Ergonomics Laboratory as a research assistant. 


\section{ACKNOWLEDGMENTS}

I would like to thank Dr. Gary Mirka, my advisor and mentor, for his invaluable counsel and advice for the last five years, and my committee members Dr. Elizabeth Loboa (co-chair), Dr. David Kaber and Dr. Peter Mente for their academic support and counsel.

I would like to acknowledge many graduate students during my Masters and Doctoral degrees for their assistance and support, and the subjects who voluntarily participated in my studies.

I would like to thank my sister Jeoung-sook and her family for their support and encouragement. Finally, I wish to express my best gratitude to my wife, Jihye, for her patience and sacrifices she made to help me achieve this goal. 


\section{TABLE OF CONTENTS}

Page

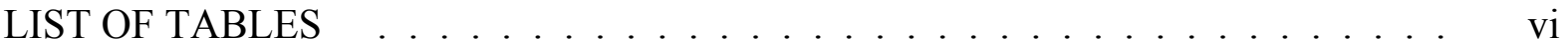

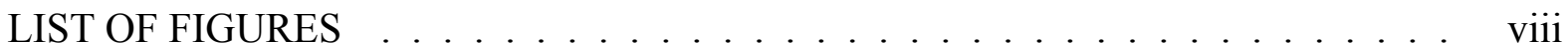

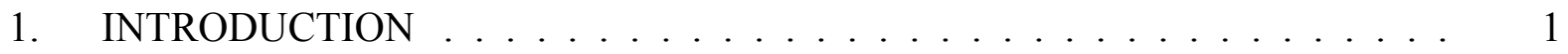

1.1. Prolonged Stooped Postures in Industry . . . . . . . . . . . . . . . . . 1

1.2. Biomechanics of the Lumbar Spine in Prolonged Stooped Postures and the Underlying Mechanisms for Low Back Disorders . . . . . . . . 3

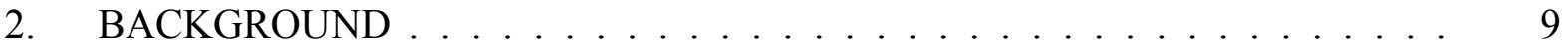

2.1. Basic and Functional Anatomy of the Lumbar Spine . . . . . . . . . . . 9

2.2. Physical Properties of the Lumbar Spine Tissues . . . . . . . . . . . . . 15

2.3. Review of Previous In Vivo Studies . . . . . . . . . . . . . . . . . . . . . . . 24

2.4. Review of Previous Finite Element Analysis Studies . . . . . . . . . . . 27

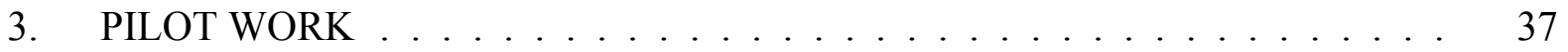

3.1. Objectives and Hypotheses . . . . . . . . . . . . . 37

3.2. Methods ....................... 37

3.3. Results ...................... 41

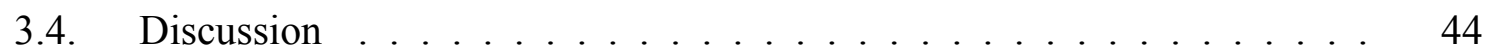

4. VALIDATION OF CONCEPT AND VERIFICATION OF METHODS . . . . . 48

4.1. Part $1 \ldots \ldots \ldots \ldots \ldots$

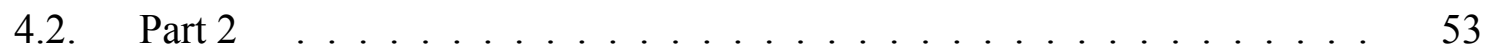

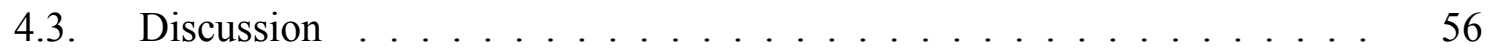

5. GENERAL OBJECTIVES AND STRUCTURE OF THE STUDY . . . . . . . . 60

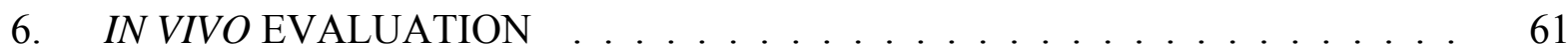

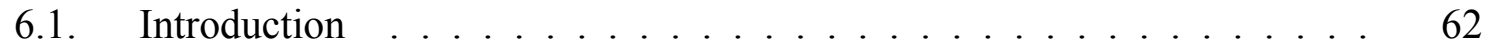

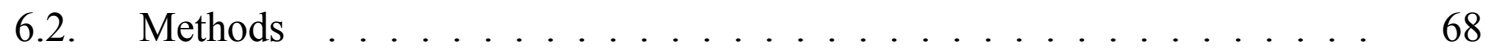

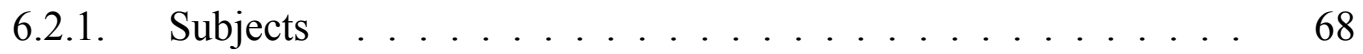

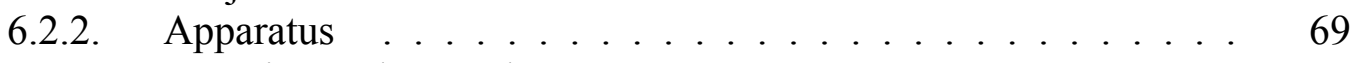

6.2.3. Experimental Procedures . . . . . . . . . . . 71

6.2.4. Experimental Variables . . . . . . . . . . . . . 74 


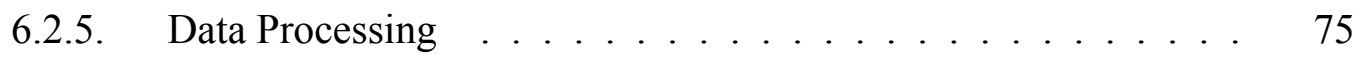

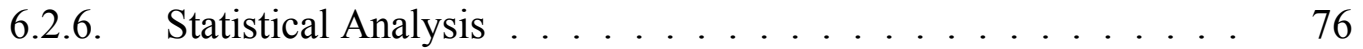

6.3. Results . . . . . . . . . . . . . . . . . . . 78

6.3.1. Full Lumbar Flexion Angle _. . . . . . . . . . . . . . . 78

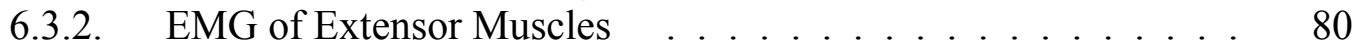

6.4. Discussion . . . . . . . . . . . . . . . . . . 84

6.5. Conclusions ....................... 91

7. FINITE ELEMENT MODEL SIMULATIONS . . . . . . . . . . . . . . 93

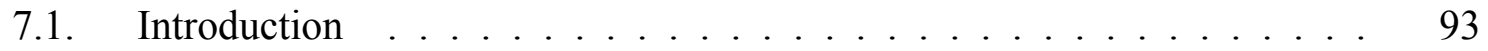

7.2. Methods ...................... 96

7.2.1. Geometry and Materials _. . . . . . . . . . . . . 96

7.2.2. Loading . . . . . . . . . . . . . . . . 106

7.3. Results . . . . . . . . . . . . . . . . 113

7.3.1. Vertical Displacement under Axial Compression Load . . . . . 113

7.3.2. Full Lumbar Flexion angle in Stooping and Recovery _. . . . 115

7.3.3. Reaction Force at Weight Holding Task . . . . . . . . . . 118

7.3.4. Hydrostatic Pressure of the Nucleus Pulposus . . . . . . . . 120

7.4. Discussion . . . . . . . . . . . . . . . . . . 122

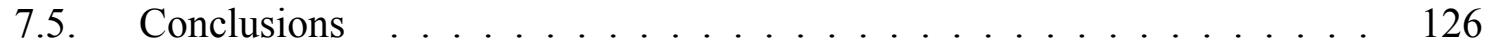

8. GENERAL DISCUSSION . . . . . . . . . . . . . . . . . . . . . . 127

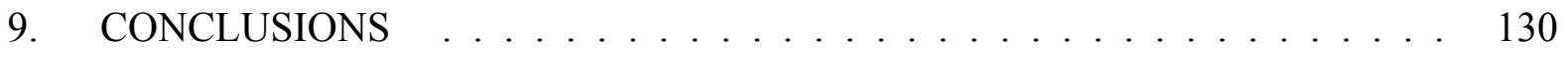

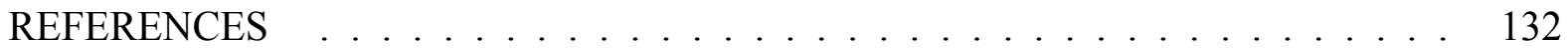

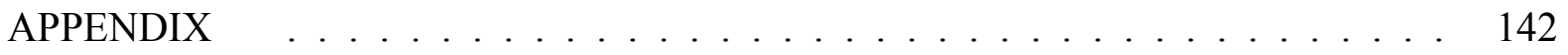




\section{LIST OF TABLES}

Page

Table 2.1. Range of motion (from peak extension to peak flexion, in degrees) of the lumbar spine joints in sagittal plane . . . . . . . . . . . . 9 9

Table 2.2. Elastic modulus of human bones . . . . . . . . . . . . . . . . 15

Table 2.3. Tensile properties of spinal ligaments . . . . . . . . . . . . . . 22

Table 6.1. Subjects' anthropometric data . . . . . . . . . . . . . . . . . 69

Table 6.2. MANOVA and ANOVA test results . . . . . . . . . . . 78

Table 6.3. Tukey's pairwise comparison between mean angle values (Means with different letters indicate they are significantly different) . . . . . . . . 79

Table 6.4. Tukey's pairwise comparison between mean EMG values (Means with different letters indicate they are significantly different) . . . . . . 81

Table 7.1. Dimensions of the vertebral body and the disc (CSA : Cross-sectional area, PCSA : Physiological cross-sectional area) . . . . . . . . . 98

Table 7.2. Element types and material properties . . . . . . . . . . . . 99

Table 7.3. Dimensions of the cross-section of the intervertebral discs in sagittal plane $(\mathrm{CSA}=$ Cross-sectional area $) \ldots \ldots$. . . . . . . . 100

Table 7.4. Prony series constants for viscoelastic elements (acquired by curve fitting to experimental data $\ldots \ldots$. . . . . . . . . . 101

Table 7.5. Loading steps (progression of simulated tasks) and boundary conditions . 109

Table 7.6. Vertical displacement of the lumbar spine in axial compression load . . . 114

Table 7.7. Pearson's correlation coefficients between the FE predicted angle and the in vivo observed angle . . . . . . . . . . . . . . . . . . . . 116

Table 7.8. Mean absolute errors between the FE predicted angle and the in vivo observed angle . . . . . . . . . . . . . . . . . . . . . . . . 116

Table 7.9. Pearson's correlation coefficients between the FE predicted force and the in vivo EMG . . . . . . . . . . . . . . . . . . . . . . . 118 
Table 7.10. Mean absolute errors between the FE predicted force and the in vivo EMG . . . . . . . . . . . . . . . . . . . . . . . . 118 


\section{LIST OF FIGURES}

Page

Figure 2.1. Disc components under compression load (from White and Panjabi, 1990) . . . . . . . . . . . . . . 11

Figure 2.2. Spinal ligaments (from 1917 Gray's Anatomy) . . . . . . . . . . . . . . 12

Figure 2.3. Lumbar vertebra (from 1917 Gray's Anatomy) . . . . . . . . . . . . . 17

Figure 2.4. Three-parameter model . . . . . . . . . . . . . . . . . . . . 19

Figure 2.5. Axial strain of the intervertebral disc under a static compression load . . 20

Figure 2.6. 2D axisymmetric FE model of the disc (from Belytschko et al., 1974) . . 29

Figure 2.7. Finite element model of the disc. (a) Sagittal cross-section; (b) mid-height section; (c) end-plate section; (d) annulus bands and fiber orientation (from Shirazi-Adl et al., 1984) . . . . . . . . . . . . 31

Figure 2.8. FE model with (a) ligaments and (b) muscles (from Zander et al., 2001) . 35

Figure 3.1. Electrode placement . . . . . . . . . . . . . 38

Figure 3.2. Normalized EMG of lumbar extensors vs. knee angle and trunk angle (no load condition) (mul : multifidus, lon : longissimus, ili : iliocostalis) .

Figure 3.3. Normalized EMG of lumbar extensors vs. knee angle and trunk angle (50\% MVC condition) (mul : multifidus, lon : longissimus, ili : iliocostalis) . .

Figure 3.4. Normalized EMG of lumbar extensors vs. flexibility and trunk angle $(50 \%$ MVC condition) (group 1 : high-flexible, group 2 : mid-flexible, group 3 : low-flexible) (mul : multifidus, lon : longissimus, ili : iliocostalis) . . . .

Figure 3.5. Normalized EMG of lumbar extensors vs. knee angle, trunk angle, and flexibility (50\% MVC condition) (mul : multifidus, lon : longissimus, ili : iliocostalis)

Figure 3.6. Simple biomechanical model showing the interaction between trunk flexion angle, knee flexion angle and individual flexibility. $\mathrm{H}$ : distance between origin and insertion of hamstring; $\mathrm{L}$ : length of a spine ligament . . . . .

Figure 4.1. MVC exertion in a lumbar dynamometer . . . . . . . . . . . 50 
Figure 4.2. Full flexion posture in a fixture $\ldots \ldots \ldots \ldots$

Figure 4.3. Mean maximum lumbar flexion angle during stooping period . . . . . . . 51

Figure 4.4. Normalized EMG at $30^{\circ}$ of trunk flexion during the weight holding tasks 52

Figure 4.5. Normailized EMG at $50^{\circ}$ of trunk flexion during the weight holding tasks 52

Figure 4.6. Mean full lumbar flexion angle . . . . . . . . . . . . . . . . . 55

Figure 4.7. Flexion-relaxation onset angle $(10 \% \max \mathrm{EMG}) \ldots \ldots$

Figure 6.1. A conceptual model relating the risk factors of prolonged stooping . . . . 67

Figure 6.2. Surface electrodes and electro-magnetic sensors . . . . . . . . . . 70

Figure 6.3. Asymmetric reference frame . . . . . . . . . . . . . . . . 71

Figure 6.4. Full lumbar flexion (stooped) posture on a pelvis restraint fixture . . . . . 73

Figure 6.5. Typical angle-EMG graph of an isokinetic lifting $\left(4^{\text {th }}\right.$ lift of stooping phase $) \ldots \ldots \ldots \ldots \ldots \ldots$

Figure 6.6. Time-dependent changes of the mean full lumbar flexion angle $\ldots \ldots 80$

Figure 6.7. Time-dependent changes of the mean EMG of multifidus.

a) average of both rest conditions, b) in each rest condition . . . . . . .

Figure 6.8. Time-dependent changes of the mean EMG of erector spinae a) average of both rest conditions, b) in each rest condition . . . . . . 83

Figure 6.9. Time-dependent changes of the stress-strain curve of a ligament during

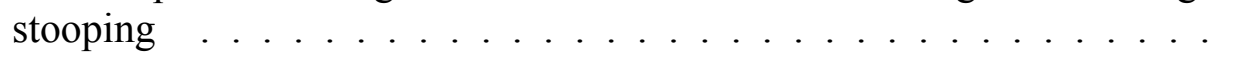

Figure 7.1. 3D FE model of lumbar spine . . . . . . . . . . . . . . . . 97

Figure 7.2. Elements for (a) ligaments and (b) muscles (shown in red) $\ldots \ldots \ldots$

Figure 7.3. Nonlinear stress-strain curves of spinal ligaments . . . . . . . . . . . 104

Figure 7.4. Normalized relaxation function of lumbar spine tissues in the model $\left(\mathrm{G}(\mathrm{t}) / \mathrm{G}_{0}\right.$ for fibers and ligaments, $\mathrm{K}(\mathrm{t}) / \mathrm{K}_{0}$ for annulus matrix $) \ldots \ldots .106$

Figure 7.5. Reference node location (a) side view, (b) front view . . . . . . . . . 107 
Figure 7.6. Boundary conditions for 'standing' load step $\ldots \ldots \ldots$. . . . . . . 108

Figure 7.7. A conceptual illustration of stress-strain curve before and after stooping . 112

Figure 7.8. Predicted full flexion angle with and without a preload . . . . . . . 114

Figure 7.9. Full lumbar flexion angle during a 10-minute stooping and a 10-minute recovery. (a) no rest condition, (b) w/rest condition (30-sec rest break at $300 \mathrm{sec}$. Not included in the duration of the stooping) . . . . . . 117

Figure 7.10. Muscle force in weight holding task (a) no-rest condition, (b) w/rest

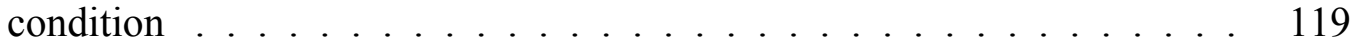

Figure 7.11. Hydrostatic pressure (MPa) of intervertebral discs after 10 minute

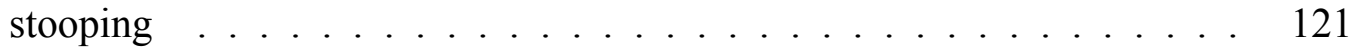

Figure 7.12. Nucleus pressure at each loading step as predicted with FE model _ . . 121

Figure 7.13. Stress of a ligament at weight holding tasks in no-rest stooping $\ldots \ldots 125$ 


\section{INTRODUCTION}

\subsection{Prolonged Stooped Posture in Industry}

Low back injuries make up a significant percentage of work-related nonfatal injuries, and these injuries are quite costly in terms of both direct and indirect costs. Work-related nonfatal injuries involving the low back were reported to be over 345,000 in 2002 (Bureau of labor statistics, 2004). Low back disorders (LBDs) are not limited to the working population as they are one of the most common disorders in the general population and affect up to $85 \%$ of the population sometime during lifetime (Korff et al., 1988; Andersson et al., 1998). LBDs can cause large economic losses such as workers' compensation costs and lost earnings from lost work days as well as uncountable costs such as pain and suffering of the injured workers and the stress on family members. In a study of workers' compensation claims of Liberty Mutual Insurance company during 1989, the total number of low back pain (LBP) cases accounted for $16 \%$ of the total number of all compensation cases and the total cost for all LBP cases accounted for $33 \%$ of the total cost of all compensable claims. Based on a generalization to other insurance companies, it was estimated that the total worker's compensation costs for LBP cases that occurred in the United States in 1989 was $\$ 11.4$ billion (Webster and Snook, 1994). Guo et al. (1999) analyzed the 1988 National Health Interview Survey to estimate the number of lost work days due to work-related back pain. The total number of lost work days as a result of work-related back pain was estimated to be 101.8 million days in the United States alone. Using the median weekly wage (\$467) in 1995, the lost earnings from lost work days due to work-related back pain was estimated to be approximately $\$ 9.5$ billion. 
LBDs can result from exposure to physical risk factors such as static awkward postures (Punnet et al., 1991; McGill, 1997), frequent bending and twisting (Marras et al., 1995; Xu et al., 1997), lifting and forceful movement (Waters et al., 1999; Kraus et al., 1997), and vibration (Pope et al., 1989). Static awkward postures include excessive sagittal trunk bending (stooped posture), lateral bending, and transverse rotation. These awkward postures are often maintained for prolonged periods of time in many occupational tasks.

The stooped posture or "stooping" is defined as "To lower the body by inclining the trunk or the head and shoulders forward, sometimes bending the knee at the same time (Oxford English Dictionary)" or "To bend forward and downward (Merriam-Webster Dictionary)." Generally, the stooped posture is performed when the task requires manual work at or below knee height for a period of time with little or no lifting/lowering of torso. A prolonged stooped posture is often seen in industries such as agriculture and construction, and this is generally considered as a risk factor that can cause LBDs (McGill, 1997; Solomonow et al., 2000). Many tasks in the agriculture and construction industries require these postures, and these industries note high levels of low back injury or illness (e.g. agricultural production field crop (Incidence Rate (IR) of back injury or illness $=45.9$ cases per 10,000 full-time workers), landscape and horticultural service ( $\mathrm{IR}=55.5$ ), roofing work $(\mathrm{IR}=91.8)$ and concrete work in construction field $(\mathrm{IR}=74.2))$. These jobs require substantial time spent in stooped postures and those tasks reported higher incidence rates of LBD compared to average industry (IR of private industry of back injury or illness $=39.1$ ) (Bureau of Labor Statistics Report, 2004). 


\subsection{Biomechanics of the Lumbar Spine in Prolonged Stooped Postures and the Underlying Mechanisms for Low Back Disorders}

The fully stooped posture requires near maximal flexion in the lumbar spine and the posture is restrained partially by extension moment from the passive spinal tissues. If the static flexed posture continues, the time variable should be included in the consideration of the biomechanical responses of the lumbar spine because of the viscoelasticity of these passive tissues. One consideration in the study of the impact of these viscoelastic effects on the lumbar spine structure during static stooped posture is the increase of the laxity or range of motion in the structure due to elongated ligaments and deformed intervertebral discs.

When a compression load is applied to an intervertebral disc, the fluid in the disc flows out thereby reducing the height of the disc and altering its biomechanical characteristics (Adams and Dolan, 1996). Lumbar flexion generates compressive load on the disc (larger load on anterior side than on posterior side) and a prolonged static flexion can cause deformation in thickness because of the biphasic fluid loss and viscoelastic strain of disc components. As the height of the intervertebral disc decreases, the space between vertebral bodies is reduced and it can result in the loss of ability to withstand compression forces and micro damage of endplates. Also the prolonged compressive loading to the disc has been shown to reduce the resistance of the spine to bending and increase the range of flexion movement in cadaver motion segment samples without ligament and muscle tissues (Adams and Dolan, 1996). When the compressive load is removed or the lumbar spine is extended to neutral standing posture, the intervertebral disc can recover its height and loadbearing abilities. Since the biomechanical responses of the disc components are nonlinear 
and viscoelastic, the deformation rate is dependent on material properties and loading history (Iatridis et al., 1996).

Another group of passive soft tissues that need to be considered during the prolonged stooped posture are the spinal ligaments. When the lumbar spine is flexed, the spinal ligaments that connect adjacent vertebral bodies (posterior side) are gradually elongated and generate a tension force. The amount of tension force generated by the passive stretch of the ligaments mainly depends on the strain rate and loading history. Similar to annulus fibers in the intervertebral disc, spinal ligaments have nonlinear and viscoelastic material properties. If the lumbar spine is flexed to a certain degree of sagittal flexion and the static posture is sustained for a long period, the tension force in these ligaments is reduced and finally reaches an equilibrium level (stress-relaxation). Ligament elongation under prolonged static flexion posture has been investigated in in vitro models and feline models but research that measured human ligament length change directly in in vivo experimentation has not been conducted. McGill and Brown (1992) investigated the creep response of the whole lumbar spine in vivo by looking at the change of peak lumbar flexion angle. They measured maximum lumbar flexion angle during 20-minute static full lumbar flexion and following 30-minute recovery phase and constructed deformation-time history curves for both phases. The peak flexion angle increased by $5.5^{\circ}$ during the 20 -minute flexion but was not fully recovered by the 30 minute recovery. They did not directly show the creep response of individual ligaments, but instead measured the "system" response to flexed postures. It was suggested that the viscoelastic behavior of the posterior tissues, especially ligaments, was responsible for the creep response of the lumbar flexion angle. 
The spinal ligaments (along with the intervertebral discs, facet joints, and muscles) are known as stabilizers of the lumbar spine, and damage to these tissues can increase the instability of the spine structure (Sharma et al., 1995). The decr3ease of stability in the lumbar spine has been known to affect the intervertebral disc pressure and the muscular activity level and finally result in pain or disorders in the low back (Solomonow, 2004). Studies investigating the relationship between spinal instability and LBD have reported that abnormal segmental motion as a cause of degeneration and/or LBD (Pope and Panjabi, 1985; Nachemson, 1985). The underlying mechanism of low back injuries associated with lumbar spine instability has been suggested by in vivo experiments and biomechanical modeling studies. Cholewicki and McGill (1996) developed a method to quantify the stability of the lumbar spine in vivo during three-dimensional dynamic tasks. In their model, muscle exertion data (established through the use of electromyography (EMG)) were used as input variables so the relationship between the muscle exertions and stability could be quantified. The model estimated that there is a sufficient stability safety margin during tasks that demand a high muscular effort, whereas lighter tasks bear a potential hazard of spine buckling and the risk increases if passive tissues lose their stiffness, which is the direct response of prolonged stooping.

In addition to these underlying mechanical mechanisms for the development of LBDs, there is also evidence for a more neurological foundation for the development of disorders in the low back during prolonged static lumbar flexion. The prolonged static lumbar flexion or cyclic flexion activities can cause micro-damage in the ligament tissues as well as the deformation in its length. Solomonow et al. (2003b) investigated the inflammatory process within the ligament tissue as a result of static flexion, by measuring neutrophil contents 
(neutrophils $/ \mathrm{mm}^{2}$ ) in ligament sections. They sampled sections of the feline supraspinous ligaments at the end of 20 minutes of static flexion (Group 1), after 20 minutes of static flexion followed by two hours of rest (Group 2), and after 20 minutes of static flexion followed by six hours of rest (Group 3). The number of neutrophils was measured from these three groups and they were compared with a control group that was not exposed to flexion or any loading. The mean neutrophil count of Group 1 was $36 / \mathrm{mm}^{2}$, which was almost the same with the neutrophil number of the control group $\left(37 / \mathrm{mm}^{2}\right)$. After two hours of rest, Group 2 showed four times increase of the neutrophil number, suggesting the inflammatory process had happened during the rest period. Finally, the group that was sampled after a six-hour rest exhibited mean neutrophil number of $4,172 / \mathrm{mm}^{2}$, which was over 100 times more than the control group and the Group 1.

The occurrence and development of an inflammation in the supraspinous ligament during the rest period could explain the unusual muscle activity patterns in the multifidus muscle that were observed by EMG. In the same study (Solomonow et al., 2003b), the lumbar spines of anaesthetized felines were subjected to static flexion by a hook that was inserted around the L4/L5 supraspinous ligament. Several levels of constant loads were applied on the supraspinous ligament for 20 minutes and a seven-hour recovery period followed immediately after the static flexion period. The EMG from the multifidus muscles and the hook displacement were collected during the recovery period as well as the static flexion loading period. When the ligament was loaded into flexion, the EMG of multifidus decreased exponentially and reached a steady state level near $45-50 \%$ of the initial activity, suggesting possible decrease in the stability of the lumbar spine due to reduced muscle force. In addition to the reduced EMG in the static loading period, the multifidus muscles showed 
random and unpredictable spasms regardless of applied load levels and time. Since muscle spasms have been known as a muscular response to tissue damage to maintain the stability and protect damaged tissues, the authors have speculated that the development of the microdamage in the ligament was a source of the muscle spasms in the static loading period. The recovery period followed the 20-minute static flexion loading and the EMG of multifidus showed three remarkable responses. In the first hour of the recovery period, the EMG showed a sharp increase and then decreased again to the level of the activity at the end of the 20-minute flexion period, while the majority of the creep of the supraspinous ligament was recovered at the same period. This hyperexcitability of the multifidus with the recovery of the ligament creep suggested that the muscles generated more forces to protect the damaged ligament by increasing stability or compensating for the lost tension in the ligament during the ligament was recovering most of its tension capability. Once the initial hyperexcitability was over, the EMG increased with an exponential like pattern during the rest of the recovery period and the magnitude of the activity level was up to three times greater than the initial hyperexcitability. This delayed hyperexcitability resulted from the development of inflammation in the supraspinous ligament as they observed during the recovery period, and the model that the authors developed predicted that the recovery in the EMG to normal levels could occur with 48 hours of rest. Since the creep and inflammation of the ligament is not fully recovered in 24 hours, the lumbar spine requires more activation of multifidus muscles to maintain the stability and protect the damaged tissues even after a full day rest. A similar task in the next day could result in more creep deformation and inflammation in the ligament because of cumulative exposures to static flexion loading, and finally make the lumbar spine more vulnerable to acute or chronic injury. 
In addition to the effects in the passive tissues, prolonged stooping can induce changes in the active extensor mechanism (extensor muscles) as well. Continuous or repeated passive stretch of human muscles leads to reduced passive tension and muscle length increase, and these viscoelastic changes can result in reduced motor unit activation/EMG and reduced force-generating capacity (Fowles et al., 2000; Avela et al., 2004). Reduced force-generating capacity of back extensors after prolonged stooping can intensify the damage in the passive tissues and lumbar instability since the muscles are required to generate more exertion to compensate for the loss of passive tissue strength and to protect the damaged tissues. 


\section{BACKGROUND}

\subsection{Basic and Functional Anatomy of the Lumbar Spine}

The lumbar spine is in the low back region and is comprised of five vertebral bodies (L1 through L5) with other soft tissues such as discs, ligaments, and muscles. The range of motion of each level of the lumbar spine has been measured by radiographic measurements (Pearcy et al., 1984; Hayes et al., 1989), videographic measurements (Gracovetsky et al., 1995) in in vivo maximum flexion-extension motion, and by direct measurements using cadaver samples (Yamamoto et al., 1989) (Table 2.1).

Table 2.1. Range of motion (from peak extension to peak flexion, in degrees) of the lumbar spine joints in sagittal plane.

\begin{tabular}{cccccc}
\hline & L1/L2 & L2/L3 & L3/L4 & L4/L5 & L5/S1 \\
\hline Pearcy et al., 1984 & 13 & 14 & 13 & 16 & 14 \\
Hayes et al., 1989 & 7 & 9 & 10 & 13 & 14 \\
Gracovetsky et al., 1995 & 8.1 & 10.9 & 12 & 11.3 & 11.2 \\
Yamamoto et al., 1989 & 10.7 & 10.8 & 11.2 & 14.5 & 17.8 \\
\hline
\end{tabular}

The intervertebral disc is located between adjacent vertebral bodies and it mainly bears the compressive load on the spine and restrains excessive intervertebral motion. Each disc consists of annulus fibrosus, nucleus pulposus, and cartilaginous end plates. The nucleus pulposus is located in the center (inner) region of the disc and it occupies $30-50 \%$ of total cross sectional area of the disc (Markolf and Morris, 1974). The nucleus is primarily water (about 70-90\%) and the percentage of water decreases with age (Iatridis et al., 1996). Its location varies along the spine segments but generally it is located in posterior side of the 
disc in the lumbar spine (White and Panjabi, 1990). The nucleus pulposus is surrounded by the annulus fibrosus. The annulus fibrosus is made of concentric layers of bands with annulus fibers embedded. The annulus consists of 15 to 25 distinct layers depending on the spine level and age. The thickness of each layer varies circumferentially and radially and increases with age (Marchand and Ahmed, 1990). The annulus fibers are oriented about $30^{\circ}$ from horizontal plane and alternate in adjacent layers (i.e. one layer is $30^{\circ}$, then the next layer is negative $30^{\circ}$, etc.) (Marchand and Ahmed, 1990). When a compression load is transmitted to the disc, the nucleus pulposus distributes the pressure laterally through its highly incompressible fluid, and the pressure, which is a form of tensile stress, is resisted by the annulus fibrosus (Figure 2.1). Cartilaginous end-plates are located at each end (superior and inferior) of the disc and separate the disc (nucleus and annulus) from adjacent vertebral bodies. Nutrition is supplied to intervertebral disc from vertebral bodies through the vertebral endplates. 


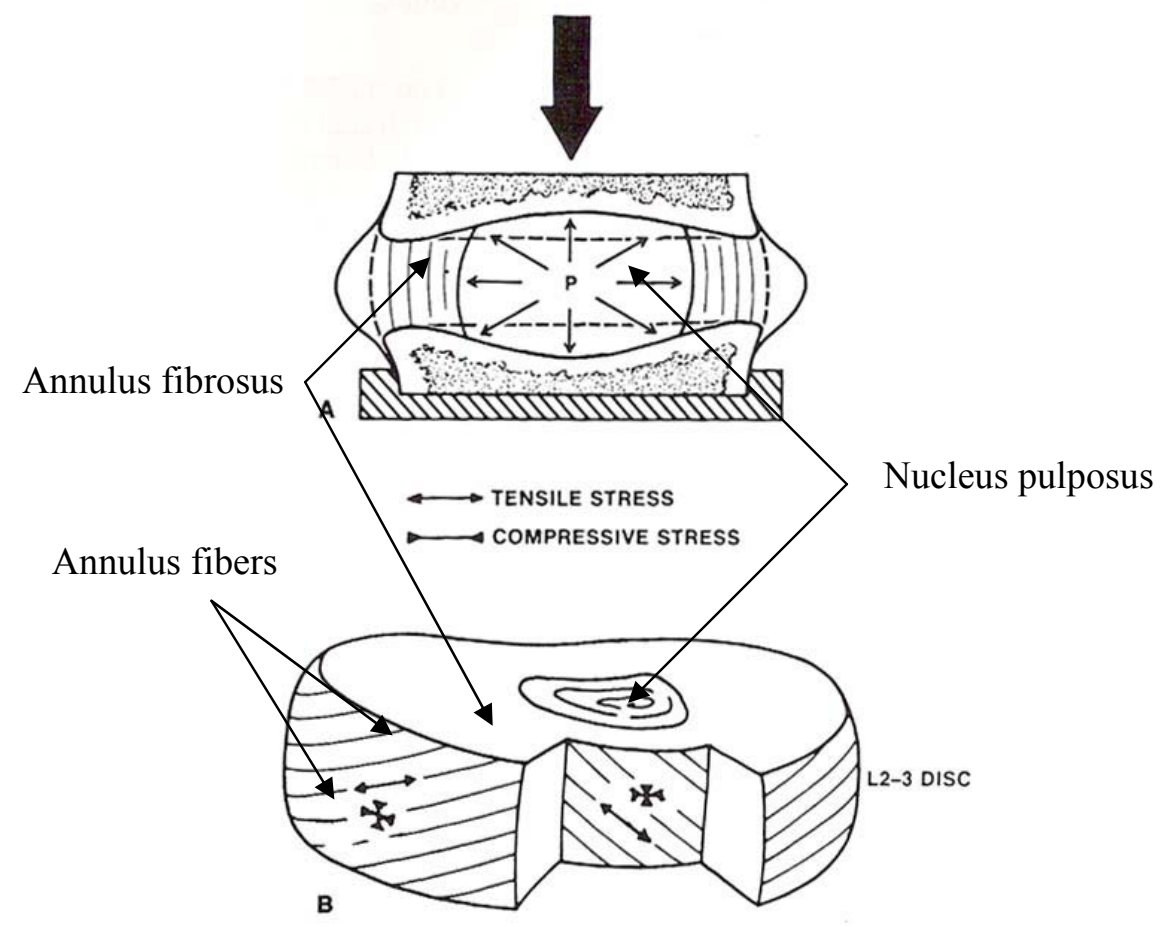

Figure 2.1. Disc components under compression load (from White and Panjabi, 1990).

Spinal ligaments are located on the anterior side (anterior longitudinal ligament) and posterior side of vertebral bodies (posterior longitudinal ligament, capsular ligament, ligament flavum, supraspinous ligament and interspinous ligament) (Figure 2.2). The anterior longitudinal ligament and the posterior longitudinal ligament are thin and broad ligaments, and they are attached to the anterior surface and posterior surface (within the vertebral canal) of the vertebral bodies and discs, respectively. They both extend from the body of the axis to the sacrum (Neumann et al., 1992). The ligament flavum on each side extends from the anterior surface of the superior lamina to the posterior edge of the inferior lamina between adjacent vertebrae. It is a continuous structure on both left and right sides (White and Panjabi, 1990; Nachemson and Evans, 1968). The supraspinous ligament 
connects spinous processes from $\mathrm{C} 7$ to sacrum. The interspinous ligament connects adjoining spinous processes and extends from the root to the apex of each process. The intertransverse ligament runs between the transverse processes. The functions of the spinal ligaments include limiting the range of motion to protect the spinal cord, providing stability of the spine structure, and supporting extensor muscles in weight holding or lifting due to passive stretching (White and Panjabi, 1990; Gracovetsky et al., 1981; Dolan et al., 1994).

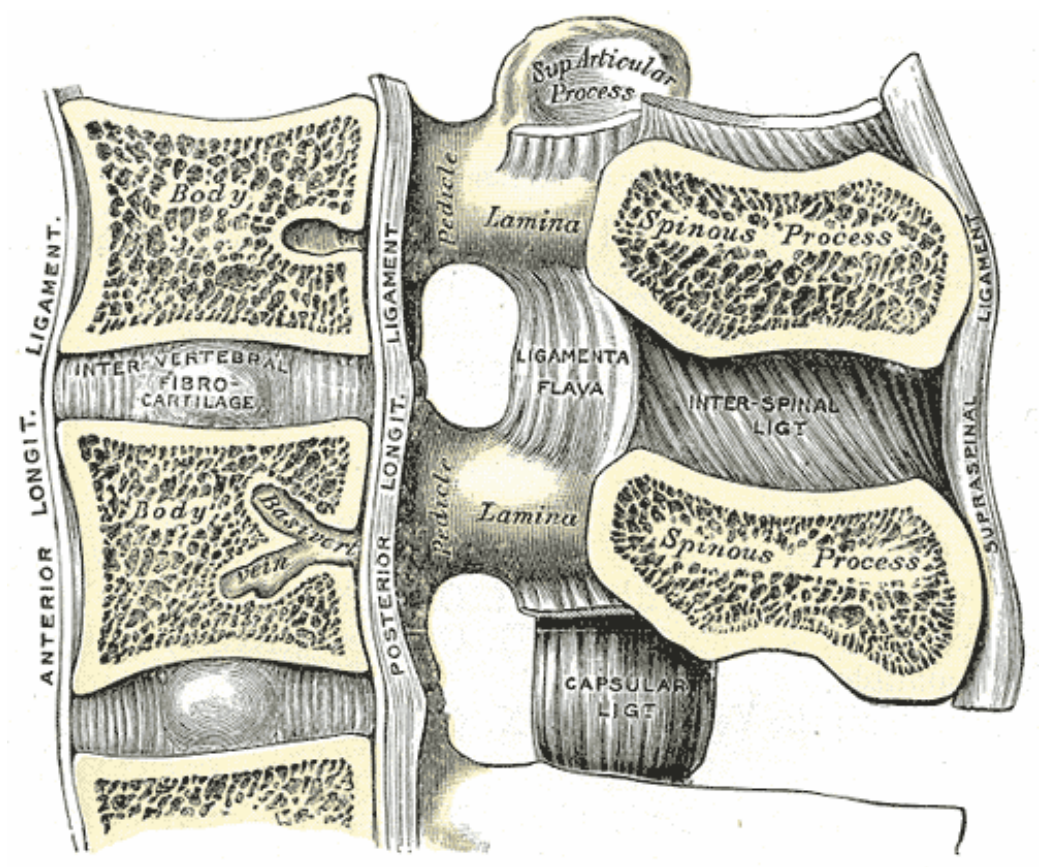

Figure 2.2. Spinal ligaments (from 1917 Gray’s Anatomy).

The active tissues of the lumbar spine include a large number of muscle groups that generate back extension moment and stabilize the spine. The lumbar erector spinae muscle group consists of iliocostalis lumborum and longissimus thoracis. They are located lateral to the multifidus muscles in the lumbar region and are generally recognized as the primary back 
extensor muscles. The longissimus thoracis (pars lumborum) originates from the transverse and accessory processes of L1 to L5 and inserts into the ventral surface of the posterior superior iliac spine. The iliocostalis lumborum (pars lumborum) arises from the tips of lumbar transverse processes and the adjacent middle layers of thoracolumbar fascia and inserts into the ventral edge of the iliac crest (Macintosh and Bogduk, 1987). They generate mainly extensor moment and posterior shear forces on the superior vertebra (McGill, 2002). Mean physiological cross-sectional area (PCSA) of the iliocostalis and longissimus fascicles were measured as $158.2(\mathrm{SD} 36.7) \mathrm{mm}^{2}$ and $99.8(\mathrm{SD} 14.9) \mathrm{mm}^{2}$, respectively (Bogduk et al., 1992). The multifidus is the largest muscle in the lumbosacral junction and is located in the most medial side. The multifidus muscles arise from the tip of the spinous process of each lumbar vertebra and insert into the posterior spines of adjacent vertebrae or posterior surface of sacrum. Their line of action is more parallel to the vertebral axis than other extensors in sagittal plane and some of them run anteriorly if they are between adjacent lumbar vertebrae. The main function of the multifidus muscles is to produce extensor moments. The PCSA of mutifidus fascicles was reported as 76.54 (SD 51.88) $\mathrm{mm}^{2}$ (Bogduk et al., 1992). In addition to these extensors, the latissimum dorsi connects lumbar spinous processes via the lumbosacral fascia and the humerus and also generates extensor moments but the contribution to extension of the lumbar spine is very small compared to other extensors (Bogduk et al., 1998). Another muscle that arises from the lumbar spine is the psoas major. The psoas major consists of overlapping fascicles that originate from the lateral and posterior sides of the discs of T12/L1 and L4/L5, transverse processes of L1-L5, and from the L5 vertebral body (Bogduk et al., 1992). From its origin, each fascicle runs downwards, laterally and forwards to reach the psoas tendon. The psoas major generates small extension 
or flexion moments in the lumbar spine because of small moment arms of fascicles but exerts substantial compression and shear loads on the lumbar spine. Instead, the psoas major is known to work as a hip flexor and a spine stabilizer (Bogduk et al., 1992; McGill, 2002).

In addition to the extensor muscles there are also a number of muscles on the anterior of the torso in the lumbar region that provide flexion moment and stability. The major abdominal muscles include the rectus abdominis and the external/internal oblique muscles. The rectus abdominis is a long flat muscle and partitioned into sections. It extends along the whole length of the front of the abdomen. The rectus abdominis is a major trunk flexor and it also tenses the abdominal wall and raises intra-abdominal pressure. The mean PCSA of the rectus abdominis has been reported in the range $360-850 \mathrm{~mm}^{2}$ (Takashima et al., 1979; McGill, 1996; Stokes and Gardner-Morse, 1999). The external oblique is a broad, thin muscle and is the most superficial and the largest of the neighboring three flat muscles (external/internal obliques, transvers abdominis). It arises from the anterior side of ribs 5 to 12, and runs downward and backward. The action of the external oblique includes raising intra-abdominal pressure and abducting and rotating trunk. The PCSA was measured as 9601,600 $\mathrm{mm}^{2}$ (Takashima et al., 1979; Stokes and Gardner-Morse, 1999; McGill, 1996). The internal oblique is located at the lateral and anterior parts of the abdomen, and beneath the external oblique. The internal oblique originates from the thoracolumbar fascia and anterior two thirds of the iliac crest, and runs downward and medially. Its action is to support the abdominal wall, raise intra-abdominal pressure, and flex and rotate the trunk. Its PCSA was in the range 1,090-1,950 $\mathrm{mm}^{2}$ (Takashima et al., 1979; Stokes and Gardner-Morse, 1999; McGill, 1996). 


\subsection{Physical Properties of the Lumbar Spine Tissues}

\section{Vertebral body and bony elements}

The vertebral body consists of cortical shell and cancellous core and the strength of the two components has been examined separately. Galante et al. (1970) measured the ultimate compressive strength (highest point before failure) of cancellous bone samples of L3 and L4 vertebral bodies and examined the relationship between the strength and bone density. The ultimate compressive strength increased linearly with the increase of the density and the average ultimate compressive strength of their 17 samples was $2.05 \mathrm{MPa}$ (SD 0.25 $\mathrm{MPa}$ ). A stress-strain relationship was not examined due to limitations of their equipment. The age information of their samples was not reported. The elastic modulus of the cancellous bone of the human vertebral body was reported by Mosekilde et al. (1987). They collected 42 bone samples from the central part of the L1 vertebral body from cadavers aged 15-87 years and examined mechanical properties of the samples under compression load. The elastic modulus of the samples was $67 \pm 45 \mathrm{MPa}$ and the ultimate strength was $2.4 \pm 1.6 \mathrm{MPa}$.

The cortical bones have greater elastic modulus and strength than the cancellous bones. Table 2.2 lists the elastic modulus of human cortical bones under compression load.

Table 2.2. Elastic modulus of human bones.

\begin{tabular}{cccc}
\hline Authors & Bone & Age (years) & Elastic modulus (MPa) \\
\hline Burstein et al., 1976 & Femur & $20-39$ & 18,350 \\
Yamada, 1970 & Femur / Tibia & $20-39$ & $17,250 / 18,030$ \\
Bayraktar et al., 2004 & Femur & $54-85$ & 19,900 \\
\hline
\end{tabular}


Anisotropy of the elastic modulus of compact bone (cortical shell) has been observed frequently (Evans, 1972; Bonfield and Grynpas, 1977). Bonfield and Grynpas (1977) tested the compressive elastic modulus (E) of mature bovine femur cortical bone specimens oriented at various angles from $0^{\circ}$ to $90^{\circ}$ to the bone long axis. The $\mathrm{E}$ values at $0^{\circ}$ and $90^{\circ}$ were reported as $17,300-19,700 \mathrm{MPa}$ and $8,700-10,300 \mathrm{MPa}$, respectively. The ' $\mathrm{E}_{\text {at } 0} / \mathrm{E}_{\text {at }} 90^{\circ}$ ' ratio was 2.3 (dry bone) or 1.7 (wet bone). Most of this variation in E occurred in two regions: $0^{\circ}-20^{\circ}$ and $70^{\circ}-90^{\circ}$.

A vertebra consists of the vertebral body and posterior elements including articular, transverse, and spinous processes (Figure 2.3). The vertebral body is a cylindrical shape bone with a cancellous bone in the middle and a thin shell of cortical bone that surrounds the cancellous bone. Elastic moduli of the three bones have been reported in FEA studies and most frequently used values are 12,000 $\mathrm{MPa}, 100 \mathrm{MPa}$, and 3,500 MPa for cortical shell bone, cancellous core, and posterior bone, respectively (Shirazi-Adl et al., 1986; Goel et al., 1993; etc). 


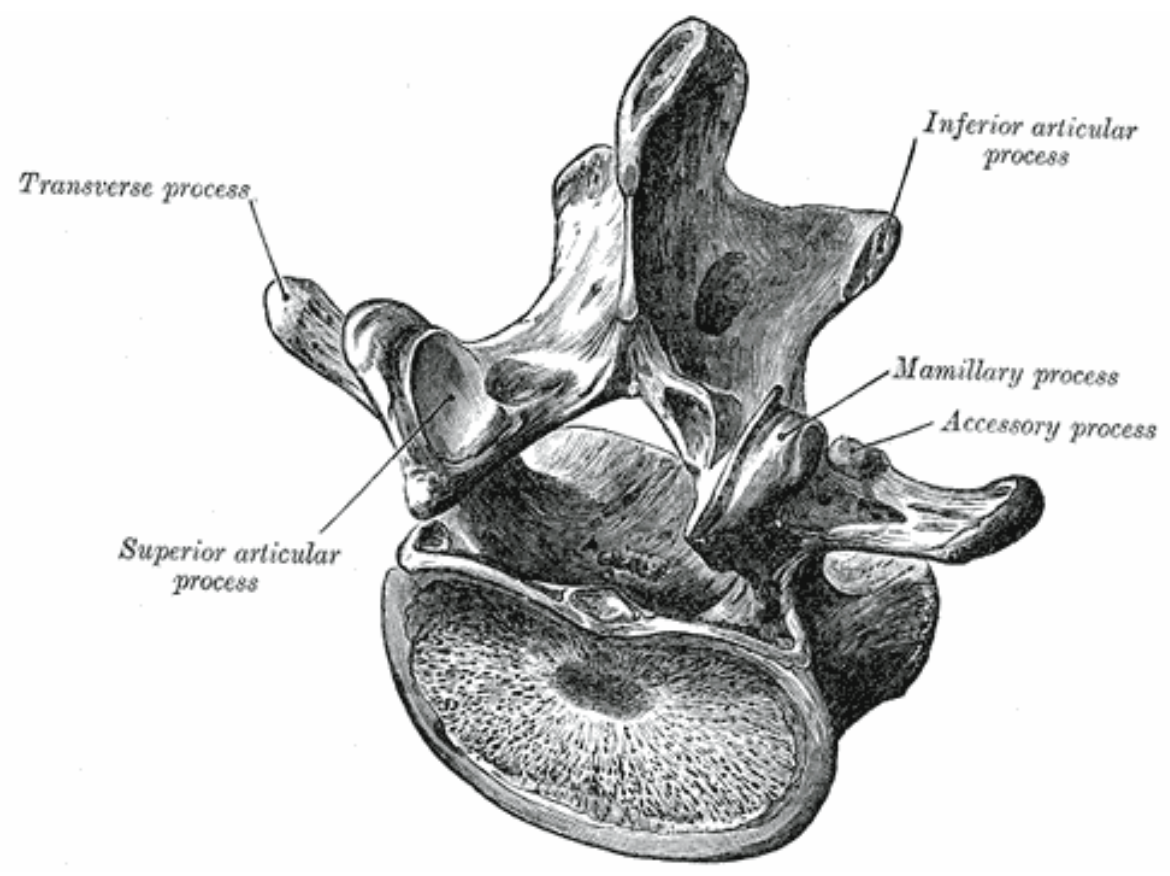

Figure 2.3. Lumbar vertebra (from 1917 Gray’s anatomy).

\section{$\underline{\text { Intervertebral disc }}$}

Physical properties of the intervertebral discs have been investigated in terms of individual components of the disc. The nucleus pulposus has been considered as an incompressible fluid because of its high content of water (70 to $90 \%$ ), and exhibits a fluid like nature under a transient load condition (slow deformation rate). However, under dynamic loading conditions, the nucleus pulposus showed a solid-like viscoelastic behavior with a stress-relaxation relationship similar to other soft tissues such as ligaments and the average shear modulus was measured as $0.011 \mathrm{MPa}$ (Iatridis et al., 1996).

The annulus fibrosus has collagen fibers in layers so the tensile properties of the annulus fibrosus are affected by the fiber orientation or load direction. Galante (1967) tested the tensile properties of annulus fibrosus samples of lumbar spine in two orientations: 
horizontal (parallel to the mid plane of the intervertebral disc) and fiber direction. Under 0.245 MPa stress, the strains in the horizontal direction and the fiber direction were observed as 0.111 and 0.115 , respectively, and they were not significantly different. Ultimate strength was $8.83 \pm 1.08 \mathrm{MPa}$ (fiber direction) and $3.43 \pm 0.29 \mathrm{MPa}$ (horizontal direction). Time dependent effects (creep property) were also tested by looking at the stress-relaxation curve. The initial slope of the curve was $-0.312 \pm 0.012 \mathrm{~N} / \mathrm{sec}$ and the slope after 60 seconds was $0.0033 \mathrm{~N} / \mathrm{sec}$. Skaggs et al. (1994) isolated a single lamella (fiber embedded in a single layer of annulus matrix) from the annulus fibrosus of lumbar spine and examined its tensile properties. The single lamella exhibited nonlinear behavior in the stress-strain relationship. The "toe-region" was observed at strain level less than $3 \%$ and then a "linear region" occurred. The tensile elastic modulus was higher in anterior-outer region than posteriorinner region. The average modulus over all area was reported as $88 \mathrm{MPa}$.

The time-dependent nonlinear deformation of the whole intervertebral disc under a prolonged static compression load was studied by Burns et al. (1984) and Li et al. (1995). Burns et al. (1984) examined the creep response of 47 human intervertebral discs that were removed from four male cadavers ranging in age from 27 to 46 years, by applying a constant axial compressive load $(177.9 \mathrm{~N})$ for seven to nine hours. The observed strain-time data points were best fitted by the three-parameter solid model for viscoelastic material (Equation 2.1). Elastic moduli $E_{1}$ and $E_{2}$, and the viscoelasticity coefficient $\mu$ are related to the coefficient of elasticity and damping by the equations in Figure 2.4. 
$\varepsilon(t)=\sigma_{0}\left[\frac{E_{1}+E_{2}}{E_{1} E_{2}}-\frac{1}{E_{1}} \exp \left(-\frac{E_{1}}{\mu} t\right)\right]$

The mean parameter values of lumbar intervertebral discs were $9.41 \mathrm{MPa}$ for $E_{1}, 8.55 \mathrm{MPa}$ for $E_{2}$, and $16,860 \mathrm{MPa} \cdot \mathrm{S}$ for $\mu$.

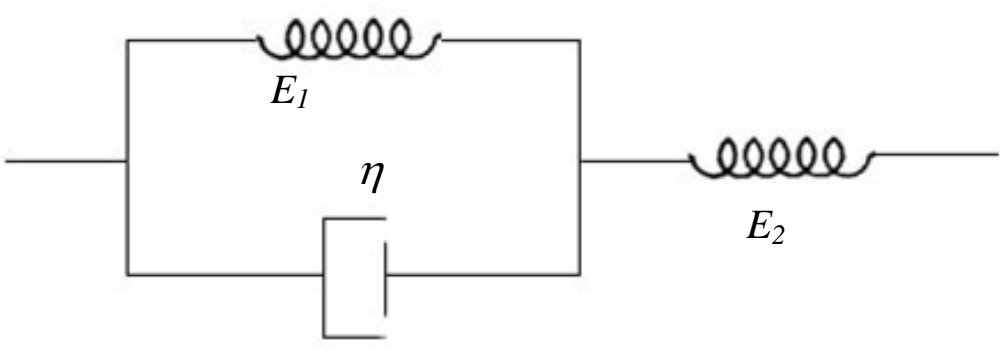

Figure 2.4. Three-parameter model.

Similarly, Li et al. (1995) isolated eight lumbar disc segments (L1/L2 and L3/L4) and eight thoracic disc segments from male cadavers ranged in age from 30 to 65 years and performed a creep test. The amount of the axial compression load ranged from $200 \mathrm{~N}$ at T5/T6 to $450 \mathrm{~N}$ at L3/L4 disc level. The load was applied incrementally from zero to the desired level in the average time of 22.9 seconds. The creep loading continued for one hour while the axial displacement of the superior vertebral body was measured. The creep response was observed during the constant loading period and the strain-time relationship was expressed using a three-parameter model. The computed parameters from their mild-degenerated samples were 9.25 (SD 1.61) $\mathrm{MPa}, 6.25(1.59) \mathrm{MPa}$, and $27.4(8.46) \mathrm{GPa} \cdot \mathrm{S}$, respectively. Figure 2.5 shows an estimated creep response curves with a $0.72 \mathrm{MPa}$ axial compression stress. 


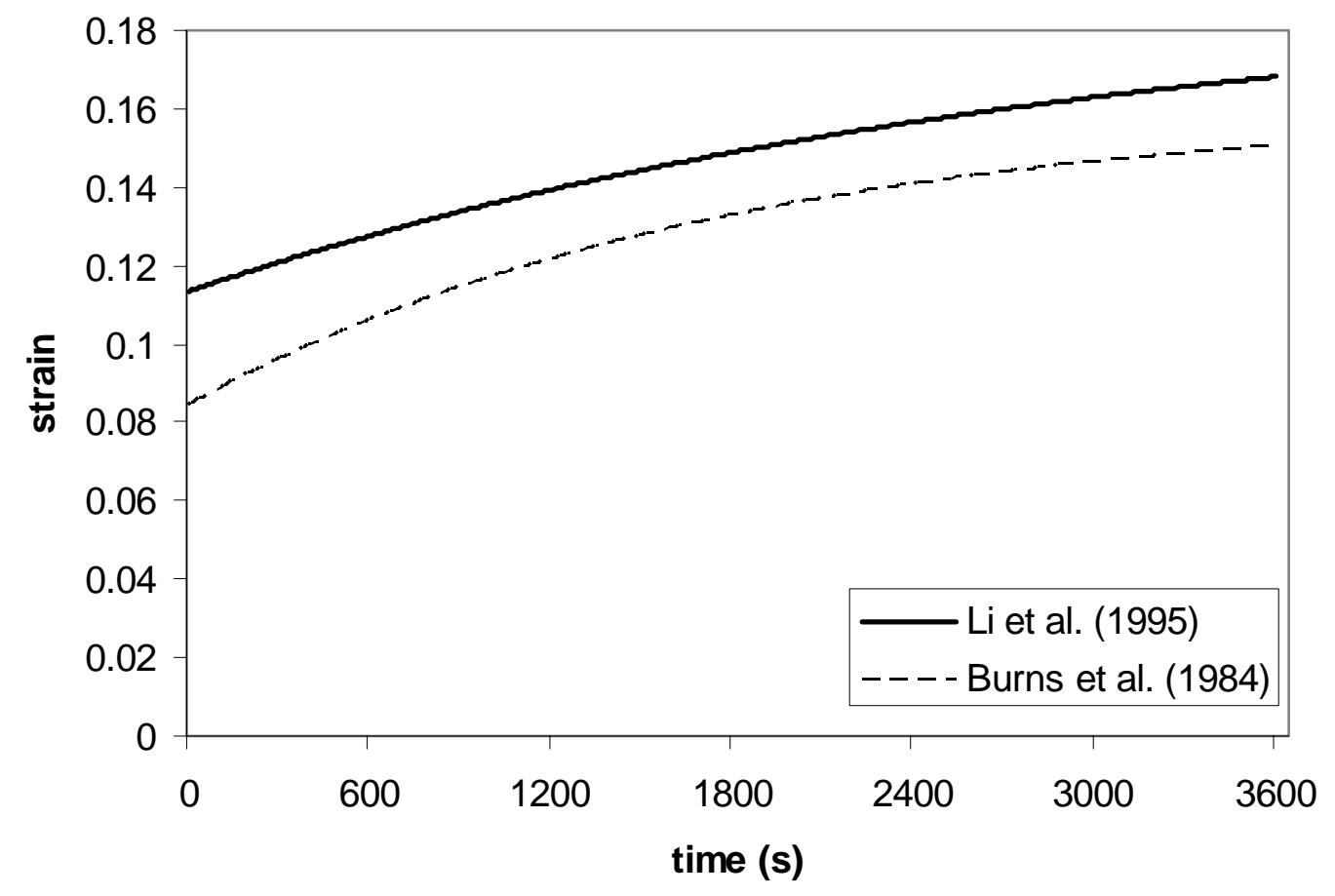

Figure 2.5. Axial strain of the intervertebral disc under a static compression load.

\section{Spinal ligaments}

Physical properties of lumbar spinal ligaments have been studied mainly in vitro. Physical material properties such as cross-sectional area, elastic modulus, and failure stress or strain significantly vary depending on test method and age of cadavers.

One of the earliest studies on spinal ligament properties was conducted by Nachemson and Evans (1968). They examined the load-strain relationship of ligament flavum samples (from L3/L4 segments) by applying multiple steps of tensile load and measuring corresponding strain of each tensile load step. Two important findings of their study were the effect of age on mechanical properties (failure load, tensile elastic modulus at failure) and the nonlinear strain-stress relationship. The elastic modulus and load at failure 
decreased linearly with age (tensile elastic modulus at failure $(\mathrm{MPa})=106.5-1.139 *$ AGE; failure load $(\mathrm{N})=19.12-0.1667 * \mathrm{AGE})$, indicating a large age effect. The profile of the stress-strain relationship had two elastic linear areas; low slope under 50\% strain and high slope over 60\% strain. Decades later Chazal et al. (1985) and Myklebust et al. (1988) performed extensive tests for tensile strength of spinal ligaments. Both studies examined the anterior longitudinal ligaments, posterior longitudinal ligaments, ligamentum flavum, interspinous ligaments, capular ligaments, and supraspinous ligaments from C2 to S1 level. Their load-deformation curves showed similar shape (sigmoid shape) as presented by Nachemson and Evans (1968). The nonlinear strain-stress relationship in the Chazal's study indicated that the slope (stress/strain) increased up to $625 \%$ from low strain region $(0.06-0.2)$ to high strain region (0.15-0.45). Average data of cross-sectional area $\left(\mathrm{mm}^{2}\right)$ and failure load (N) of each ligament are listed in Table 2.3. 
Table 2.3. Tensile properties of spinal ligaments.

\begin{tabular}{|c|c|c|c|c|c|c|}
\hline \multirow[t]{2}{*}{ Name } & \multirow[t]{2}{*}{ Level } & \multicolumn{2}{|c|}{ Chazal et al. (1985) } & \multicolumn{2}{|c|}{ Pintar et al. (1992) } & \multirow{2}{*}{$\begin{array}{c}\text { Myklebust et al. (1988) } \\
\text { Failure load (N) }\end{array}$} \\
\hline & & $\begin{array}{l}\text { Failure load } \\
\text { (N) }\end{array}$ & $\begin{array}{c}\text { CSA } \\
\left(\mathrm{mm}^{2}\right)\end{array}$ & $\begin{array}{l}\text { Failure load } \\
\text { (N) }\end{array}$ & $\begin{array}{c}\text { CSA } \\
\left(\mathrm{mm}^{2}\right)\end{array}$ & \\
\hline \multirow{5}{*}{$\mathrm{AL}$} & L1-L2 & 500 & 44 & 434.2 & \multirow{5}{*}{32.4} & 341 \\
\hline & L2-L3 & - & - & 521.6 & & 504 \\
\hline & L3-L4 & 482 & 72 & 414.7 & & 676 \\
\hline & L4-L5 & 545 & 70 & 511.9 & & 606 \\
\hline & L5-S1 & - & - & 265.7 & & 209 \\
\hline \multirow{5}{*}{ PL } & L1-L2 & 390 & 20 & 59.8 & \multirow{5}{*}{5.2} & 91 \\
\hline & L2-L3 & 437 & 31.3 & 147.7 & & 160 \\
\hline & L3-L4 & 313 & 26.3 & 63.4 & & 38 \\
\hline & L4-L5 & - & - & 107.1 & & 53 \\
\hline & L5-S1 & - & - & 102.4 & & 108 \\
\hline \multirow{5}{*}{$\mathrm{LF}$} & L1-L2 & - & - & 210.5 & \multirow{5}{*}{84.2} & 215 \\
\hline & L2-L3 & - & - & 109.5 & & 133 \\
\hline & L3-L4 & 340 & 39 & 244.2 & & 251 \\
\hline & L4-L5 & - & - & 244.2 & & 334 \\
\hline & L5-S1 & - & - & 345.2 & & 287 \\
\hline \multirow{5}{*}{$\mathrm{SS}^{*}$} & L1-L2 & - & - & 390.6 & \multirow{5}{*}{25.2} & 378 \\
\hline & L2-L3 & - & - & 249.5 & & 742 \\
\hline & L3-L4 & 278 & 31.5 & 317.5 & & 750 \\
\hline & L4-L5 & 230 & 34.25 & 320.0 & & 329 \\
\hline & L5-S1 & 115 & 18.5 & 352.8 & & 293 \\
\hline \multirow{5}{*}{ IS } & L1-L2 & - & - & 207.1 & \multirow{5}{*}{35.1} & 185 \\
\hline & L2-L3 & - & - & 63.2 & & 98 \\
\hline & L3-L4 & - & - & 63.2 & & 95 \\
\hline & L4-L5 & - & - & 101.8 & & 115 \\
\hline & L5-S1 & - & - & 193.1 & & 118 \\
\hline \multirow{5}{*}{$\mathrm{CL}$} & L1-L2 & - & - & 451.1 & \multirow{5}{*}{43.8} & 429 \\
\hline & L2-L3 & - & - & 630.7 & & 299 \\
\hline & L3-L4 & - & - & 337.3 & & 290 \\
\hline & L4-L5 & - & - & 153.3 & & 353 \\
\hline & L5-S1 & - & - & 245.3 & & 326 \\
\hline
\end{tabular}

AL : anterior longitudinal ligament / PL : posterior longitudinal ligament / LF : ligament flavum / SS : supraspinous ligament / IS : interspinous ligament / CL : capsular ligament

*SS and IS were measured together in Chazal et al. (1985) 
Another important physical property of the spinal ligaments is viscoelasticity. The viscoelastic properties of the posterior ligaments in the lumbar spine have not been investigated thoroughly as most previous tests looked for stress or strain at failure by stretching ligaments until failure point or permanent plastic deformation. One of the few studies investigating these viscoelastic properties was conducted by Yahia et al. (1991). They performed a hysteresis experiment, stress-relaxation and stepwise load-relaxation tests to examine the viscoelastic properties of the interspinous-supraspinous ligament complex in the human lumbar spine. The stress-relaxation relationship was expressed as a linear function of the natural logarithm of time (Equation 2.2), which was originally proposed by Fung (1981). Equilibrium was reached after 5,000 seconds and half of the final relaxation occurred during the first 10 seconds.

$\mathrm{G}(\mathrm{t})=0.95-0.044 \ln (\mathrm{t})\left(\mathrm{r}^{2}=0.992\right)$

where, $\mathrm{G}(\mathrm{t})$ is the reduced relaxation function (stress at time ' $\mathrm{t}$ ' $=\mathrm{G}(\mathrm{t}) *$ initial stress)

\section{Muscle-tendon units}

Muscles can also generate a passive tension force when stretched and the passive force increases in an exponential fashion as the muscle is stretched beyond its unstressed length (Hawkins and Bey, 1997). Studies with in vivo animal muscles or human lower extremity muscles estimated that the maximum tensile passive force in fully flexed posture could reach up to twice of the maximum active force of the muscle (Gareis et al., 1992; Hawkins and Bey, 1997). The maximum force per unit area of the erector spinae of in vivo 
human has been reported as $0.48 \mathrm{~N} / \mathrm{mm}^{2}(0.48 \mathrm{MPa})$ (Reid and Costigan, 1987). The ultimate tensile strength of human cadaver muscles of people 20 to 39 years of age ranged from 0.1 MPa to $0.3 \mathrm{MPa}$ but, with the consideration of the rapid postmortem decrease in the ultimate strength of muscle tissue, the maximum stress of the erector spinae muscles at fully flexed posture could be estimated to be between 0.5 and $1 \mathrm{MPa}$. Stress-relaxation and creep deformation of muscle-tendon units in repetitive or prolonged passive stretch were also observed (McHugh et al., 1992; Taylor et al., 1997) but the quantitative relationship of the viscoelastic responses of the muscle-tendon units were not provided.

\subsection{Review of Previous In Vivo Studies}

The idea that the passive tissues help the back extensors generate extension moments during lifting or weight holding originated from the flexion-relaxation phenomenon. When the back starts flexing from the neutral standing posture, the back extensor muscles generate force (through an eccentric exertion) and provide the extension moment supporting the mass of the upper body. At the same time, the posterior ligaments and other passive tissues are strained and gradually generate extension moment by passive resistant forces. As the flexion angle increases, the passively generated moment takes over from back extensors as a main contributor to support the body weight. The myoelectrical activity of back extensors, beyond this point, approaches zero. This electric silence at full flexion is often called 'flexionrelaxation' phenomenon because the back extensor muscles appear to be in a relaxed state. Under this fully flexed posture the lumbar spine ligaments and the passive component of the muscular tissues are believed to be stretched enough to generate the required extension moment to maintain static equilibrium. This 'flexion-relaxation' depends more on lumbar 
spine flexion than the flexion of the overall trunk angle (Kippers and Parker, 1984; Floyd and Silver, 1955). The contribution of the passive tissues to generating extension moments has also been observed during lifting motions. Dolan et al. (1994) assessed the passive extensor moment during lifting. Their subjects performed isometric upward pull tests with steadily increasing force on a floor-mounted load cell while EMG in erector spinae muscles were recorded. The relationship between the EMG and the extensor moment (calculated from the load cell data) was linear with the intercept (at ' 0 ' EMG) on the moment axis which indicated the portion of the moment resisted by passive components. They computed that between 16 and $31 \%$ of the peak extensor moment during the pull test was unrelated to the EMG activity in the erector spinae, and less than a quarter of this passively generated extensor moment came from the intervertebral discs and ligaments.

The effects of the viscoelastic properties of passive tissues of the lumbar spine structure on the flexion-relaxation angle have been studied in several in vivo experiments. Dickey et al. (2003) compared the onset angle of the flexion-relaxation and the maximum flexion angles before and after 100 repetitive lifts with and without an external load. The increase of the flexion-relaxation onset angle and the maximum flexion angle were observed for both loading conditions but only the change in the no-load condition was statistically significant. The angle was greater with load at pre-lifts (before repetitive lifts) but it did not differ between the load and no load conditions after the repetitive lifts (post-lifts). This result was not consistent with their hypothesis that the flexion-relaxation angle would be larger in the load condition than no load condition, regardless of repetitive lifts. They concluded that the change in the neuromuscular reflex system, which was suggested by Eversull et al. (2001), might be occurring during the repetitive lifts. This thought was based on the idea that the 
flexion-relaxation is the result of balance between the external flexion moment and the passive extension moment by the posterior ligaments, and the increase of the external moment (in the load condition) requires the balancing activity of the erector spinae until the passive extension moment is increased at greater flexion angle. The larger flexion-relaxation angle with load condition was observed in several studies (Kippers and Parker, 1984; Gupta, 2001). Different from these studies, Dickey et al. (2003) measured the flexion-relaxation angle before and after repetitive lifts, which might cause significant fluid loss in the intervertebral discs and make the discs stiffer than before repetitive lifts. The increase of maximum flexion angle and flexion-relaxation angle in the no-load condition could be simply explained as the result of reduced thickness of intervertebral discs. When the external load is added at pre-lifts the compression load on the intervertebral discs reduces disc thickness and finally results in the increase of the flexion angle. However, when the load is applied at post-lifts the intervertebral discs have become stiff already and do not allow additional deformation by the added load, especially the load is not enough to make them stiffer and requires more ligament elongation.

The flexion-relaxation response to prolonged static lumbar flexion was also examined by Solomonow et al. (2003a). They collected EMG of erector spinae (L3-L4 level) and lumbar flexion angle by video-based motion capture system $(60 \mathrm{~Hz})$ simultaneously to identify the flexion-relaxation onset (EMG-off) and ending (EMG-on) angles and the maximum voluntary lumbar flexion angle before, after, and during static full flexion posture (sitting on a flat mat in a full lumbar flexion position) for 10-minute period. They found a significantly greater flexion angle for both EMG-on and EMG-off after the 10-minute static flexion and the effect was stronger in females. Muscle spasms were also observed in more 
than half of the subjects during the static flexion period, suggesting some micro-damage occurred in the ligaments. The increase of flexion-relaxation angle after prolonged static flexion period implied that a 10-minute lumbar flexion caused creep of passive tissues and the muscles compensate for the reduced tension in the lumbar spine passive tissues.

The creep of passive tissues in the lumbar spine and its influence on the back extensor muscle activities has been rarely investigated. Most previous studies have focused on the effects of creep on the flexion-relaxation angle. A review of the literature found no direct in vivo (human) experimental results that have attempted to quantify the effects of creep on the extensor muscle activity levels in postures somewhat less than the full flexion angle. Understanding this response could add insight into the tradeoffs between the passive and active components of the extensor mechanism and how creep affects this relationship.

\subsection{Review of Previous Finite Element Analysis Studies}

Finite Element Analysis (FEA) is a numerical technique to obtain approximate solutions in complex loading scenarios. It was first developed in the late 1940's for use in structural analysis in aerospace and nuclear industries. In FEA, a structure is divided into a grid or mesh of finite sized elements of simple geometric shape. The points at which neighboring elements are joined together are called 'nodes'. Elements contain material properties and governing relationships which are expressed mathematically in terms of unknown values (e.g. displacement) at nodes. With boundary conditions, the unknown values at nodes are computed by solving a set of algebraic equations. Since the discretized model has a finite number of degrees-of-freedom (DOF), the technique is called finite element analysis. 
Much of the finite element modeling of the lumbar spine region has been focused on the study of intervertebral discs. The intervertebral discs are often prone to cumulative trauma injury resulting from prolonged mechanical damage and/or degeneration. However, because the direct measurement of the load and deformation of the discs in in vivo experiments are difficult, the nature of disc disease/degeneration has not been clearly explained by in vivo experimental approaches. In order to better understand the stress and strain distributions in discs that might lead to damage under various loading conditions, finite element analysis (FEA) methods have been applied to disc modeling since the 1970's (Belytschko et al., 1974).

The first detailed FEA study of the intervertebral disc was reported by Belytschko et al. (1974). They created a two-dimensional (2D) axisymmetric model of the intervertebral disc and adjacent vertebrae under an axial compression load (Figure 2.6). The nucleus pulposus was assumed to be an incompressible fluid and the annulus fibrosus was modeled as a linear orthotropic material by varying the elastic modulus in the fiber direction. Bone was assumed to be isotropic and homogeneous with a mean elastic modulus for the cortical shell and cancellous core of the vertebral body of 15,789 $\mathrm{MPa}$ and $73.5 \mathrm{MPa}$, respectively. The cartilaginous end plate was also modeled as an isotropic homogeneous material with an elastic modulus of $23.8 \mathrm{MPa}$. The load was applied by setting a uniform vertical displacement to the top surface, and fixing the bottom surface. The model showed good correlation compared with experimental studies, and it was found that the overall mechanical behavior of the disc could not be adequately represented if the orthotropic material behavior of the annulus was not included in the model. 


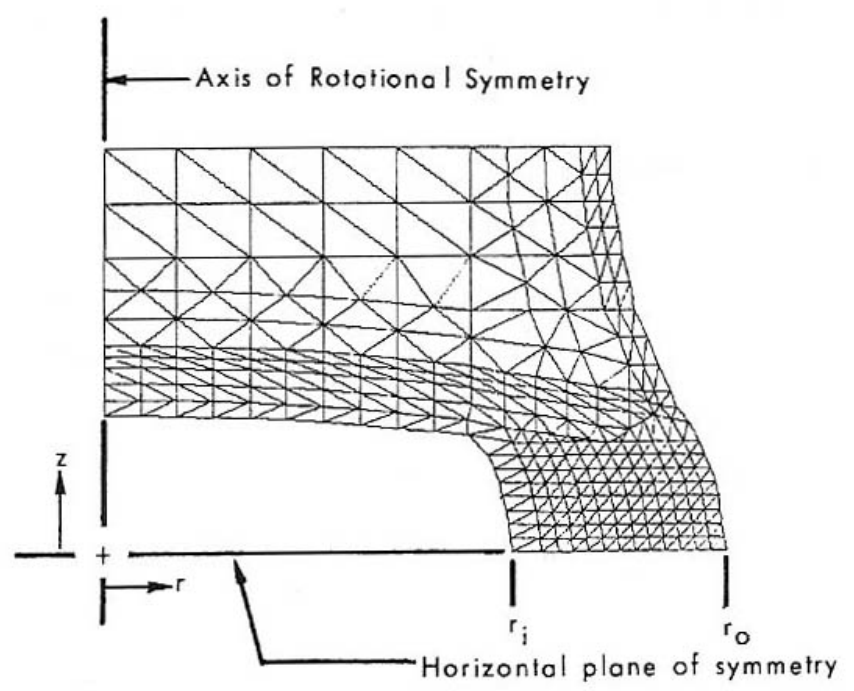

Figure 2.6. 2D axisymmetric FE model of the disc (from Belytschko et al., 1974).

Kulak et al. (1976) extended the model by considering a nonlinear time-independent relationship for the annulus. The nonlinear orthotropic material constants of the annulus fibrosus were estimated by matching the overall behavior of the disc under axial compressive loading to the experimental results. Their study suggested that the nonlinear model exhibited better agreement with experimental studies than a linear model for responses such as bulging, nuclear pressure, and deformation under various loadings and suggested that the nonlinear material behavior needs to be included in the study of the intervertebral disc over the normal in vivo range of loading. Spilker (1980) built an axisymmetric disc model with a linear isotropic annulus to examine the effects of disc geometry and material property parameters on mechanical response of the simple model of the disc and found increasing disc radius and disk height led to a decrease in nuclear pressure, disc bulge, and vertical deflection. In general, all parameters had a significant effect on disc bulge and vertical deflection, suggesting the wide variation of mechanical responses often observed in in vitro 
experimental data of the intervertebral disc. The first three-dimensional (3D) model of the intervertebral disc was introduced by Liu et al. (1975). The elastic modulus of the annulus was acquired by an optimization technique (minimizing the error between the experimental data and the predicted deformations). They found that axial compression of the disc was sensitive to both shear modulus and compressive elastic modulus of the annulus, and it is because the annulus fibrosus sustained both the vertical compression force and the horizontal shear force through the hydrostatic pressure in the nucleus pulposus.

A more realistic FEA model of the intervertebral disc was first constructed by Shirazi-Adl et al. (1984). They created a 3D model of a quarter of the disc-vertebral body unit (Figure 2.7) so the model could predict a variation in disc bulge along the annulus edge and the effect of geometric nonlinearities resulting from large deformation. The model considered the nucleus pulposus as an incompressible fluid, occupying about $30-60 \%$ of the cross-sectional area of the disc. Different from previous studies, the annulus fibrosus was modeled as a composite of two different elements: annulus ground substance (8 layers) and collagenous fibers; with more detailed geometry and material properties based on previous experimental and anatomical studies being incorporated into the model. The fibers were embedded in each layer of the annulus at an average angle of $29^{\circ}$ to the horizontal plane. The variation of the cross-sectional area and elastic property of fibers along the radius of the annulus were also considered to incorporate nonlinear properties of the annulus. Validation of their model showed good agreement with experimental reports in terms of intradiscal pressure and end-plate deformation. After this model was introduced, most following FEA studies also modeled the annulus as a composite structure with similar features to those that used by Shirazi-Adl et al. (1984). 

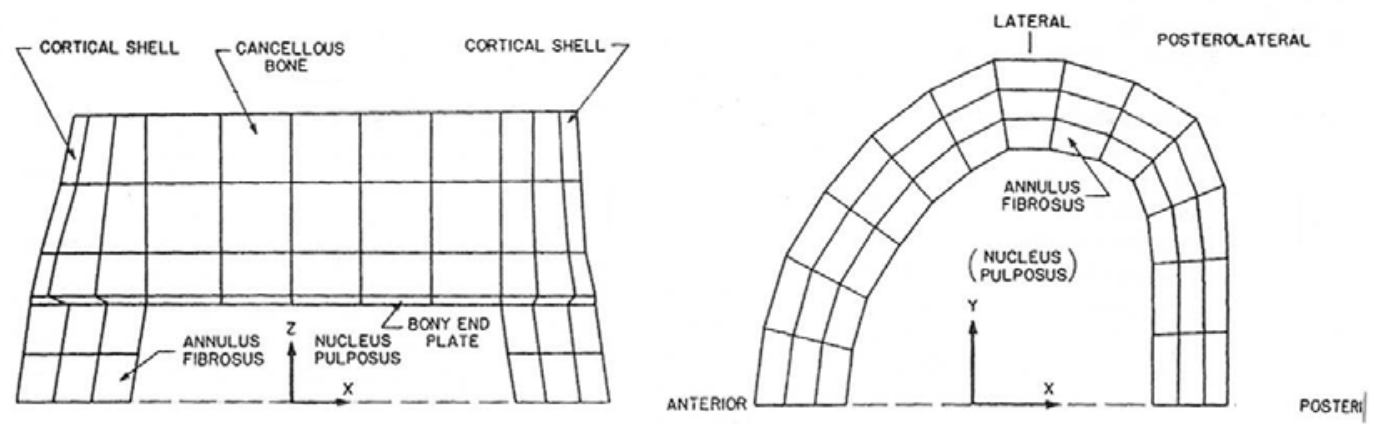

(a) $Y=0 . \mathrm{mm}$

(b) $z=0 . \mathrm{mm}$

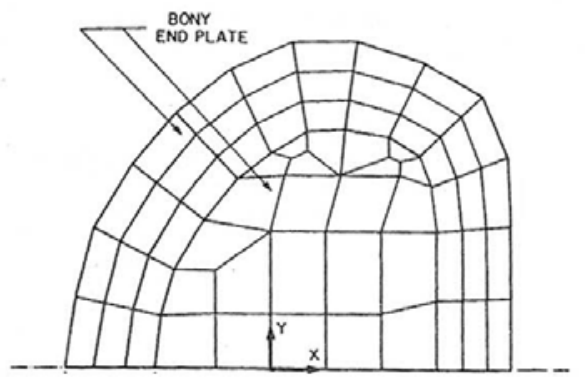

(c) $Z=5.5 \mathrm{~mm}$

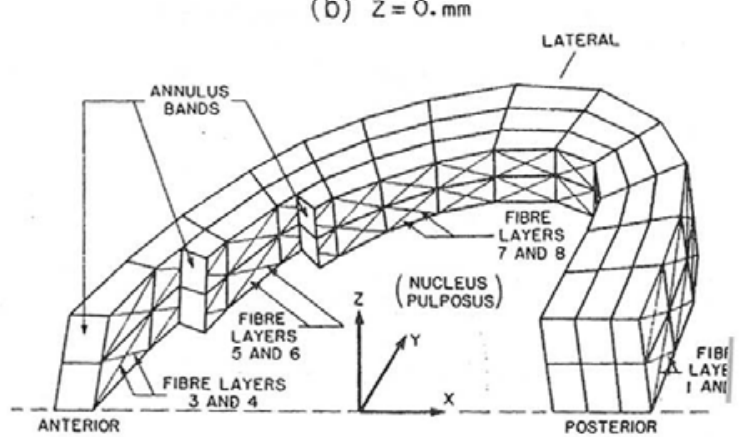

(d)

Figure 2.7. Finite element model of the disc. (a) Sagittal cross-section; (b) mid-height section; (c) end-plate section; (d) annulus bands and fiber orientation (from Shirazi-Adl et al., 1984).

In subsequent research, Shirazi-Adl et al. (1986) examined the gross response of the full motion segment under pure sagittal plane moments with a revised model. In their revised model (Shirazi-Adl et al., 1986), articulation at the facet joint was considered using a contact relationship between the facet articulation surfaces, and spinal ligaments with nonlinear material properties were also incorporated. The identical material properties were applied to all different ligaments due to the absence of published data on the material properties of the various ligaments. The posterior ligaments, in flexion, were found to be the main path that transferred the load from the upper vertebra to the lower one and the strain of some ligaments 
could reach their maximum strain (rupture point) under large flexion. Loss of disc pressure had little effects on the strain of the ligaments in flexion.

A similar model was built by Goel and Kim (1989). Their FEA model was created with identical material properties for bones and disc materials as used in Shirazi-Adl's studies, but they applied bilinear material properties to ligaments. Each ligament had two Young's moduli and they were assigned as a bilinear function of strain to represent the nonlinear stress-strain relationship of the ligament. Since they examined the mechanical responses in the disc under compression load conditions, the influence of the nonlinear stress-strain of the ligament was not evaluated.

Few FEA studies have included spinal muscles in their lumbar spine models. Goel et al. (1993) addressed this issue by incorporating the spinal muscles into a FEA model of the lumbar spine motion segment to identify the role of muscles in motion segment mechanics. They constructed a FEA model of the L3/L4 segment with ligaments and applied muscle forces on the vertebra as predicted by an optimization-based biomechanical model, and compared displacements and stresses to a no-muscle model. The muscle-incorporated model led to a decrease of the anteroposterior translation and sagittal flexion rotation in sagittal flexion loading, a decrease of intradiscal pressure, and increase of facet load in axial compression loading. These results indicated that the motion segment became more stable with the addition of muscles to the model.

A full 3D model of a whole lumbar spine was developed as a next step by Shirazi-Adl and Parnianpour (1993). They generated a full lumbar spine model (without muscles) from L1 to S1 with detailed 3D irregular geometry and nonlinearities. Geometry of the bones was created by computer-assisted tomographic (CT) images of a 65 years old cadaver, and 
material properties used for the disc, bones, and ligaments were assumed to be the same at all segmental levels due to the lack of precise information. An axial compression load was applied at the centroid of the L1 vertebral body and the S1 was fixed in all directions to examine the variation of the lumbar spine in $3 \mathrm{D}$ movements during the compressive load. Results showed highly nonlinear responses due to the lordotic geometry of the lumbar spine as well as the nonhomogeneous and nonlinear material properties, and the interaction with the surrounding tissues. Using the same model, Shirazi-Adl (1994) applied sagittal and lateral moments to the $\mathrm{L} 1$ to see the effects on disc fibers and the role of facet joints under sagittal and lateral moments in addition to general biomechanical responses of the spine. They found that large tensile strains were predicted in the disc fibers, suggesting the vulnerability of the disc fibers to failure under large flexion movements. Posterior ligaments played a significant role in sagittal flexion and the facet joints remained unloaded or slightly loaded up to a $15 \mathrm{~N}-\mathrm{m}$ flexion moment. Flexion/extension and lateral rotation angles of the entire lumbar spine predicted by this model were in the range of in vitro measured results.

As prolonged static work postures have been identified as a risk factor for low back pain, viscoelastic FEA models of the intervertebral disc or spine segment have been developed to investigate the time-dependent deformation or stress in the lumbar spine components. Recent studies that incorporated viscoelastic properties into FEA models often used the Prony series or nonlinear spring-dashpot models to simulate the viscoelastic behavior of the disc components and ligaments (Wang et al., 1997; Wang et al., 1999; Wang et al., 2000; Lu et al., 1998). Wang et al. (1997, 1999, and 2000) applied the Zener model (two springs and one dashpot) to nonlinear annulus fibers and ligaments, and the Prony series to annulus matrix and nucleus pulposus. The material property constants and the Prony 
constants were set to simulate the fluid loss in the annulus matrix and nucleus during creep loading. Different from Wang's studies, Lu et al. (1998) focused more on the fluid loss of the nucleus. Lu et al. (1998) also built a viscoelastic FEA model of an L2-L3 motion segment by simulating viscoelasticity of the annulus fibers and ligaments using the Prony series, and fluid loss of the nucleus using the three-parameter Kelvin model (two springs and a dashpot). In the simulation of creep loading, the bottom surface of the L3 vertebra was assumed to be fixed and an axial compressive load of $1000 \mathrm{~N}$ was applied within 0.001 seconds to the top surface of L2 with this force kept constant for an hour. Results suggested that the viscoelastic behavior of the disc mainly depends on the magnitude and rate of fluid loss, and the intrinsic properties of the disc components only contribute to the viscoelastic behavior of the disc at the early stage of loading.

Some recent studies predicted muscle forces in their FEA models. Zander et al. (2001) used 3D nonlinear FEA model of a whole lumbar spine to estimate back muscle forces during inclination of the upper-body, and to determine the influence of flexion angle and muscle force on the stresses in the lumbar spine (Figure 2.8). The lower endplate of the L5 vertebral body was fixed and the $400 \mathrm{~N}$ body load was applied vertically at the center of gravity of the upper body through a beam that connected with the upper endplate of the L1 vertebra. The muscles were modeled as tension-only elements, and their lines of actions and attachments were similar to those chosen by Bogduk et al. (1992). Included muscles were multifidus, iliocostalis lumborum pars lumborum, longissimus thoracis pars lumborum and longissimus thoracis pars thoracis. The same force intensity was assumed for all muscles. Results found that the stress distribution in the discs differed considerably when the muscle 
forces are neglected, indicating that muscle forces should not be neglected when studying the stresses in the lumbar spine.

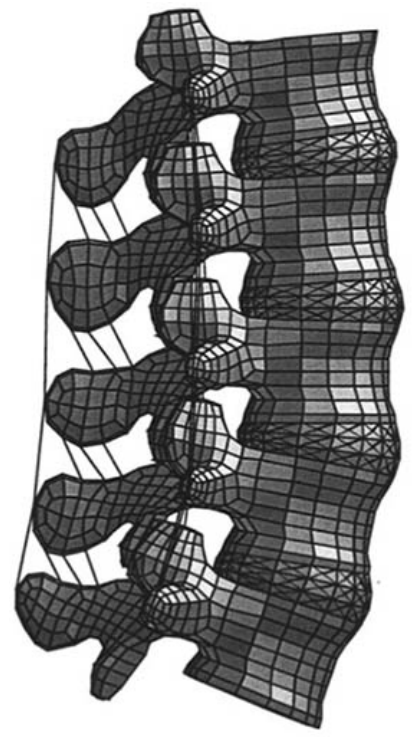

(a)

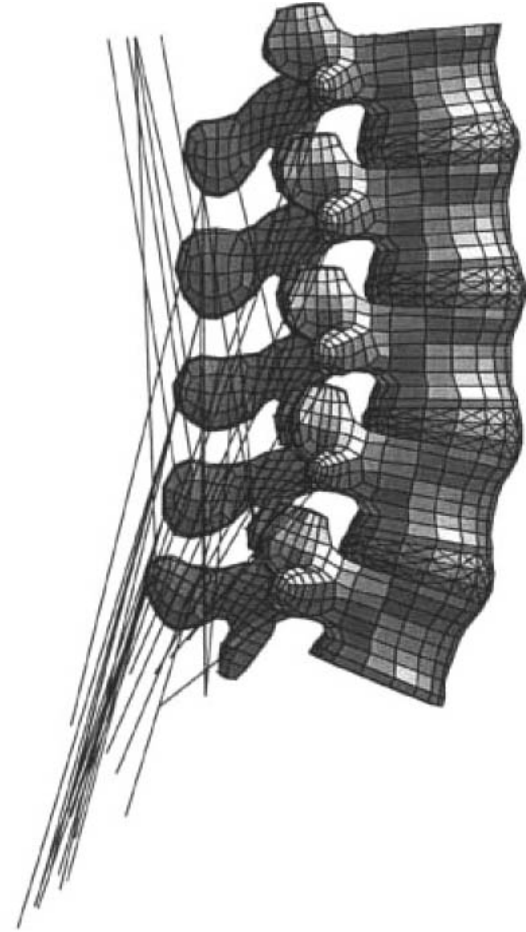

(b)

Figure 2.8. FE model with (a) ligaments and (b) muscles (from Zander et al., 2001).

For a study of prolonged stooping, a FE model of the lumbar spine should include both ligaments and extensor muscles because they are maintaining lumbar stability and supporting upper body weight with biomechanical and physiological interactions between them. The strength of the ligaments and muscles are time and posture-dependent due to the nonlinear and viscoelastic material property of the tissues. Previous FEA models have examined viscoelastic responses of the lumbar spine without muscles (Wang et al., 1997; 
Wang et al., 2000; Cheung et al., 2003; Lu et al., 1998) or elastic behaviors with muscles (Zander et al., 2001; Goel et al., 1993; Shirazi-Adl et al., 2002). No FE model of a whole lumbar spine with full nonlinear and viscoelastic material properties has been created for the analysis of mechanical responses during prolonged stooping. 


\section{PILOT WORK}

\subsection{Objectives and Hypotheses}

Objectives of this pilot work were to quantify the interactive effect of trunk angle and knee angle on the lumbar extensor activity and to quantify the effect of individual trunk flexibility in this response. Since the knee flexion angle could affect the rotation of pelvis in the trunk flexion motion, the muscle activities of back extensors were expected to change as the knee angle changes. Also the flexibility is somewhat related to the ligament tension in the flexed posture and it might influence the muscle activity level in large flexion posture. Results of this pilot work could be used to provide some empirical data that support the idea of ligament contribution to generating extension moment in weight holding tasks.

\subsection{Methods}

Eight healthy male subjects (mean age 27 years, SD of age 2.3 years) participated voluntarily. The flexibility of each subject was assessed by having him flex his trunk forward and reach towards the ground with knees straight, and the flexibility was categorized as low-flexible (finger tip reach greater than $+5 \mathrm{~cm}$ from floor, two subjects) (group 3), midflexible (finger tip reach between $-5 \mathrm{~cm}$ and $+5 \mathrm{~cm}$, three subjects) (group 2), and highflexible (finger tip reach less than $-5 \mathrm{~cm}$ from floor, three subjects) (group 1).

Surface electrodes were used to collect the electromyographic (EMG) activity of the lumbar extensor muscles (Figure 3.1). The angle-specific maximum voluntary contraction (MVC) EMG of the lumbar muscles were collected by an isokinetic lumbar dynamometer. This dynamometer system was also able to provide a measure of the angle-specific peak moment generated by the subject, necessary for the calculation of the hand-held loads. 


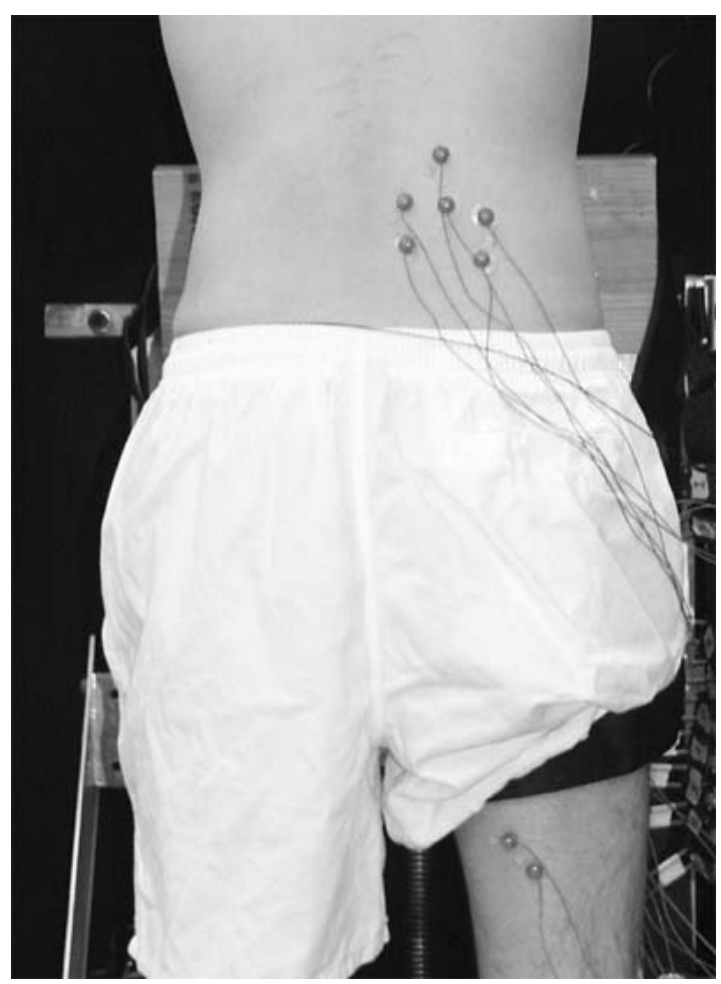

Figure 3.1. Electrodes placement.

Independent variables included sagittally symmetric trunk flexion angles $\left(30^{\circ}, 50^{\circ}\right.$, $70^{\circ}$, and $\left.90^{\circ}\right)$, knee flexion angles $\left(0^{\circ}\right.$ (knees straight), $20^{\circ}$, and $\left.40^{\circ}\right)$, static moment on L5/S1 joint (no external load, and 50\% of the subject- and angle specific back extension moment), and subject flexibility level (high, medium, low). The trunk flexion angle was defined as an angle between a vertical reference line and the line joining the acromion process and greater trochanter. The angle between the line joining the greater trochanter and the center of rotation of the knee and the line joining the center of rotation of the knee and the lateral malleolus of the ankle was defined as the knee flexion angle. Dependent variables were the normalized EMG of the lumbar extensors (multifidus, iliocostalis, and longissimus) on the right side of the vertebral column. 
Surface electrodes were placed on multifidus $(1.5 \mathrm{~cm}$ to the right of the vertebral midline at L4 level), longissimus ( $3.5 \mathrm{~cm}$ to the right of the vertebral midline at $\mathrm{L} 2$ level), and iliocostalis $(4.5 \mathrm{~cm}$ to the right of the vertebra midline at $\mathrm{L} 2$ level) with a fixed interelectrode center to center distance of $2.5 \mathrm{~cm}$. The electrode placement locations were chosen to maximize the contribution of the named muscles based on the relative cross-sectional areas of the muscles in the region (L2-L4). Prior to placement, the electrode placement area was shaved, abraded and cleansed with an isopropyl alcohol absorbed cotton ball to lower the electrical impedance. All EMG data were collected at $1024 \mathrm{~Hz}$. After the experimental setup, the maximum voluntary back extension moment and lumbar extensor MVC EMG data were collected using the isokinetic dynamometer as the subject performed the isometric back extension exertions at each of the four different trunk flexion angles $\left(30^{\circ}, 50^{\circ}, 70^{\circ}\right.$, and $\left.90^{\circ}\right)$. The MVC trials were collected only after the subject completely understood and performed consistent pre-test trials. In the subsequent trials the subject experienced loading conditions of no load (simply holding the weight of the torso) and a condition that required the subject to produce an extension moment (about $\mathrm{L} 5 / \mathrm{S} 1$ ) equal to $50 \%$ of their posture specific capacity. This $50 \%$ condition was accomplished by having the subject hold a barbell loaded with the appropriate amount of weight. The weight of the hand-held load is the function of MVC force, body weight, and body dimensions of each subject and it was calculated by the equation (3.1)

Hand-held weight $=[(50 \%$ of max. extension moment $)-($ flexion moment by upper body weight)] / moment-arm length from the center of weight to L5/S1 
The subject performed a total of 48 trials (two repetitions of all combinations of four trunk flexion angles, three knee flexion angles, and two load conditions). At each trial, the subject bent their torso forward and flexed (or straightened in the case of $0^{\circ}$ condition) both knees and held the barbell using both hands. The experimenters used goniometers to establish when the subject had achieved the appropriate knee and trunk angles. The subject was then asked to hold that position and keep his heels in contact with floor. As soon as the posture was stable, the EMG data were collected for three seconds. There was a 20 -second rest break between consecutive trials during which the subject was allowed stand up in a relaxed posture. Task order was fully randomized.

Raw EMG data were filtered in software using a $10-500 \mathrm{~Hz}$ pass filter as well as a notch filter that eliminated $60 \mathrm{~Hz}$ and its aliases. Once filtered, these signals were full-wave rectified and averaged across the three second data collection period. This processing occurred in both the data collected during the experimental trials as well as the MVC exertions. The EMG data collected during the maximum exertions were then partitioned into $1 / 8$ second windows and the maximum of the 24 windows for each muscle of each posture were identified and were used as the denominator in the process of normalizing the experimental data. The normalized EMG data set was partitioned into two subsets by load condition and then each set was analyzed using ANOVA to examine the effects of trunk flexion angle, knee flexion angle and subject flexibility and their interactions. This preliminary ANOVA was performed to get a sense of the global effects of these variables. To test the specific hypotheses of the current study further partitioning of the data was necessary. In an effort to be more refined in the analysis of the effect of flexibility and knee angle on the flexion-relaxation response, these datasets were further partitioned by trunk 
angle and an ANOVA was performed on each of these trunk angle-specific datasets to identify those particular trunk angles at which the flexion-relaxation response was influenced by knee angle and individual flexibility.

\subsection{Results}

The trunk flexion angle had a significant $(\alpha=0.05)$ effect on the lumbar extensor muscles with increased trunk flexion angle leading to decreased muscle activity in both loading conditions (Figures 3.2 and 3.3). Subjects with the greatest flexibility had the highest activity levels of the lumbar extensor musculature across trunk angles (Figure 3.4).

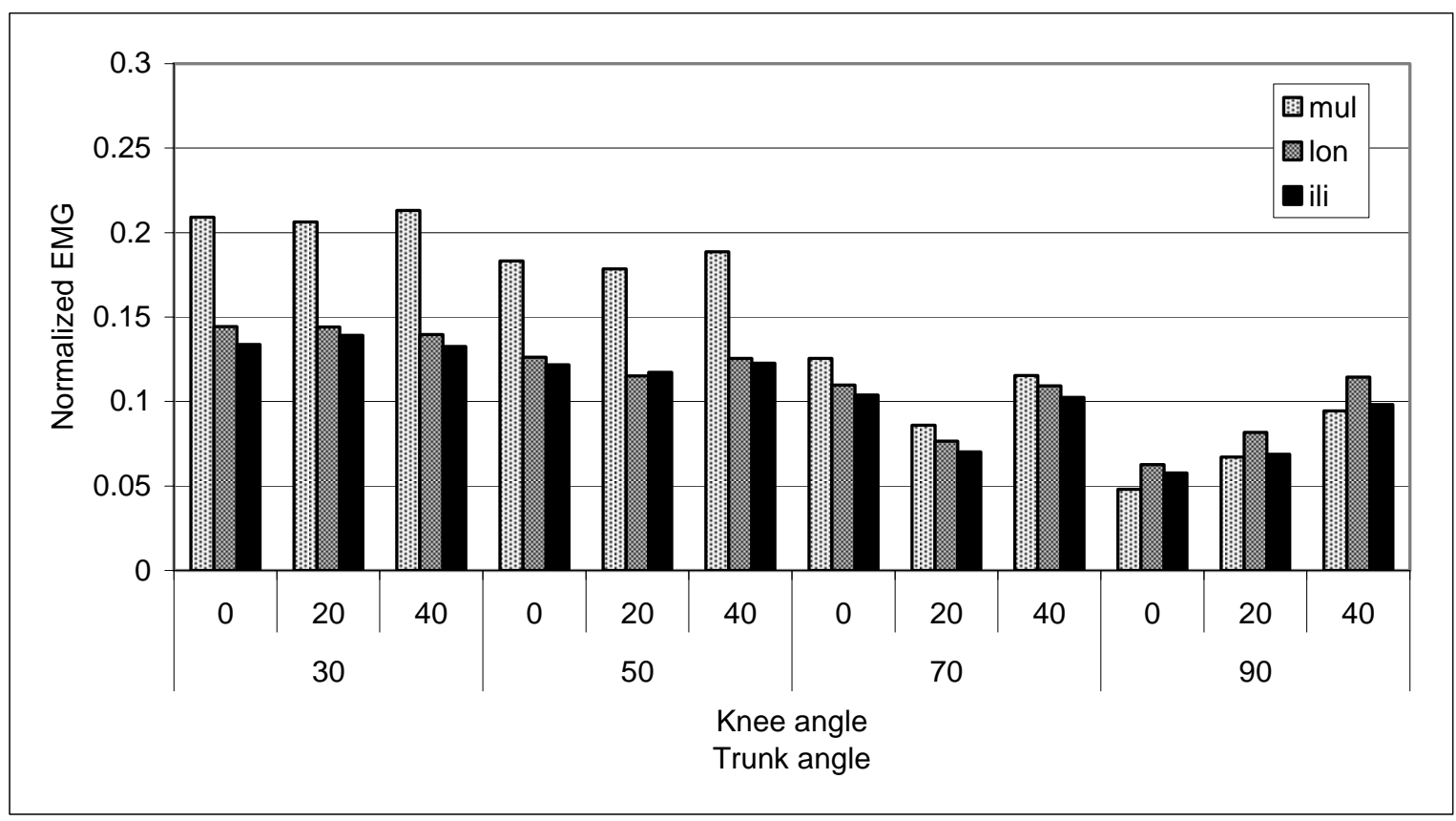

Figure 3.2. Normalized EMG of lumbar extensors vs. knee angle and trunk angle (no load condition) (mul : multifidus, lon : longissimus, ili : iliocostalis). 


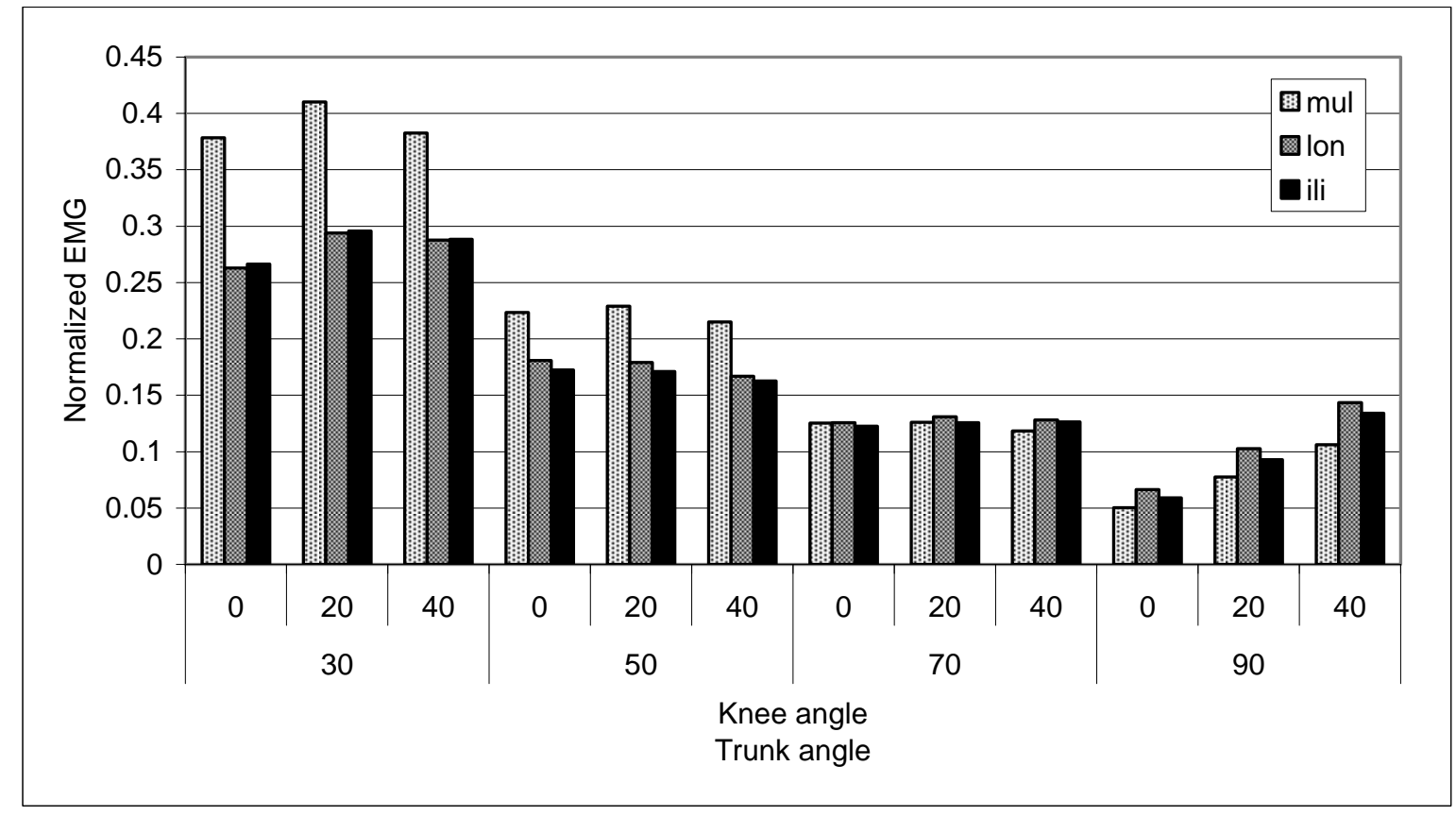

Figure 3.3. Normalized EMG of lumbar extensors vs. knee angle and trunk angle (50\% MVC condition) (mul : multifidus, lon : longissimus, ili : iliocostalis).

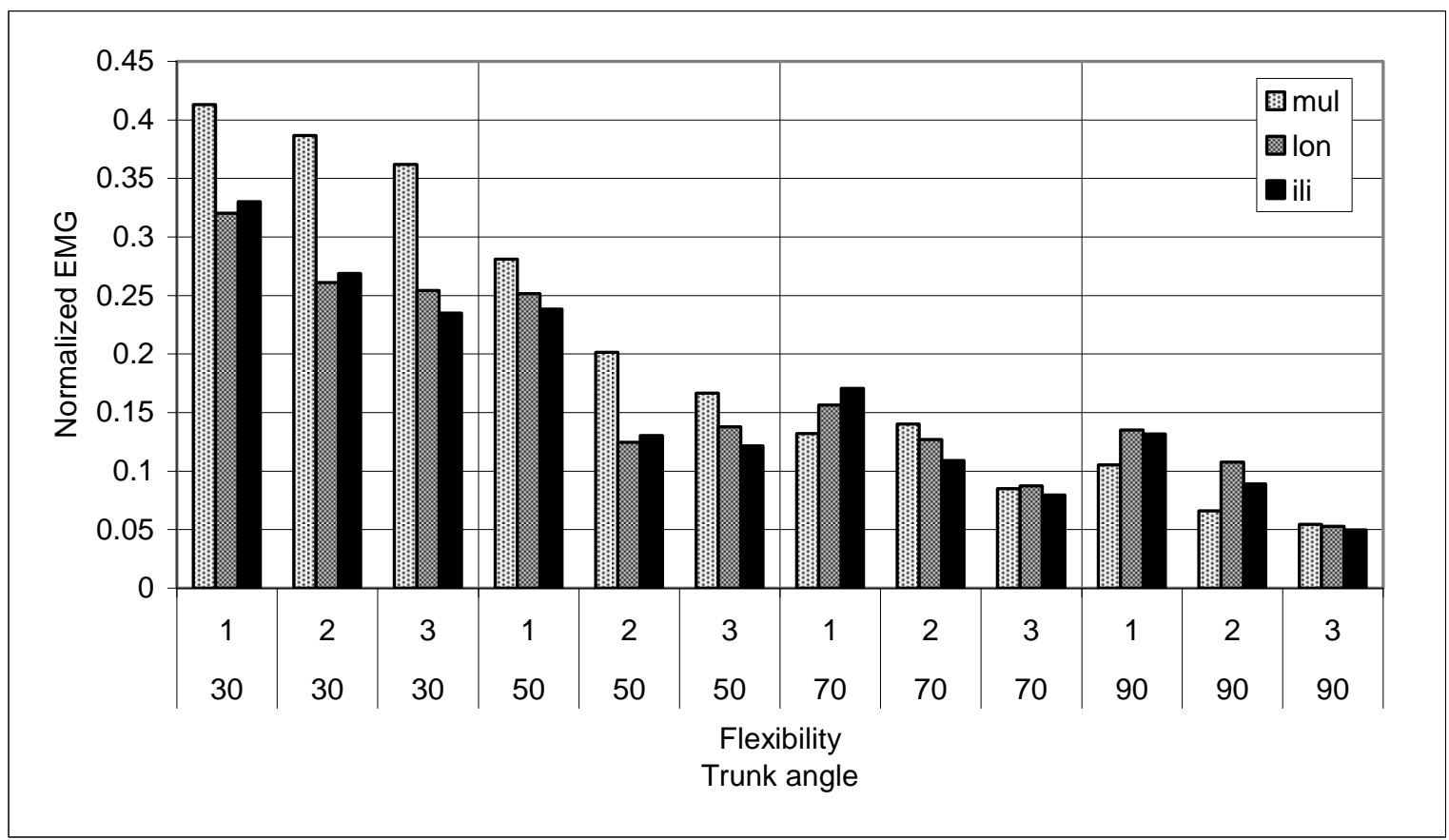

Figure 3.4. Normalized EMG of lumbar extensors vs. flexibility and trunk angle (50\% MVC condition) (group 1 : high-flexible, group 2 : mid-flexible, group 3 : low-flexible) (mul : multifidus, lon : longissimus, ili : iliocostalis). 
These results indicate that the effect of knee angle is particularly important when the subject nears the end of their range of motion where the slight changes in pelvis rotation can have a significant impact on the passive contribution to the extensor moment. On the other hand, the effect of individual flexibility appears to be a more consistent response across trunk angles. Figure 3.4 shows that at every trunk angle the low flexibility group generated less muscle activity and relied more on the passively generated extensor moment. Further exploration of this flexibility response in the extreme flexion positions $\left(70^{\circ}\right.$ and $\left.90^{\circ}\right)$ showed that the knee angle response was affected by the flexibility of the subject (Figure 3.5). This figure illustrates that for the high flexible subjects there was a strong effect of knee angle on the flexion-relaxation response at the $90^{\circ}$ trunk flexion angle but there was no knee angle effect at $70^{\circ}$ position. In the middle flexibility group it appears that this knee angle effect is also seen $70^{\circ}$ position indicating that the transition point from passive to active occurs around this point for this group. In the low flexibility group there does not appear to be any knee angle effect on the flexion relaxation process indicating that, for this group, the transition point is less than $70^{\circ}$ and regardless of knee angle the passive contribution is still high. Further post-hoc statistical analysis showed that only the EMG at $90^{\circ}$ position in the high flexibility group increased significantly $(\alpha=0.05)$. 


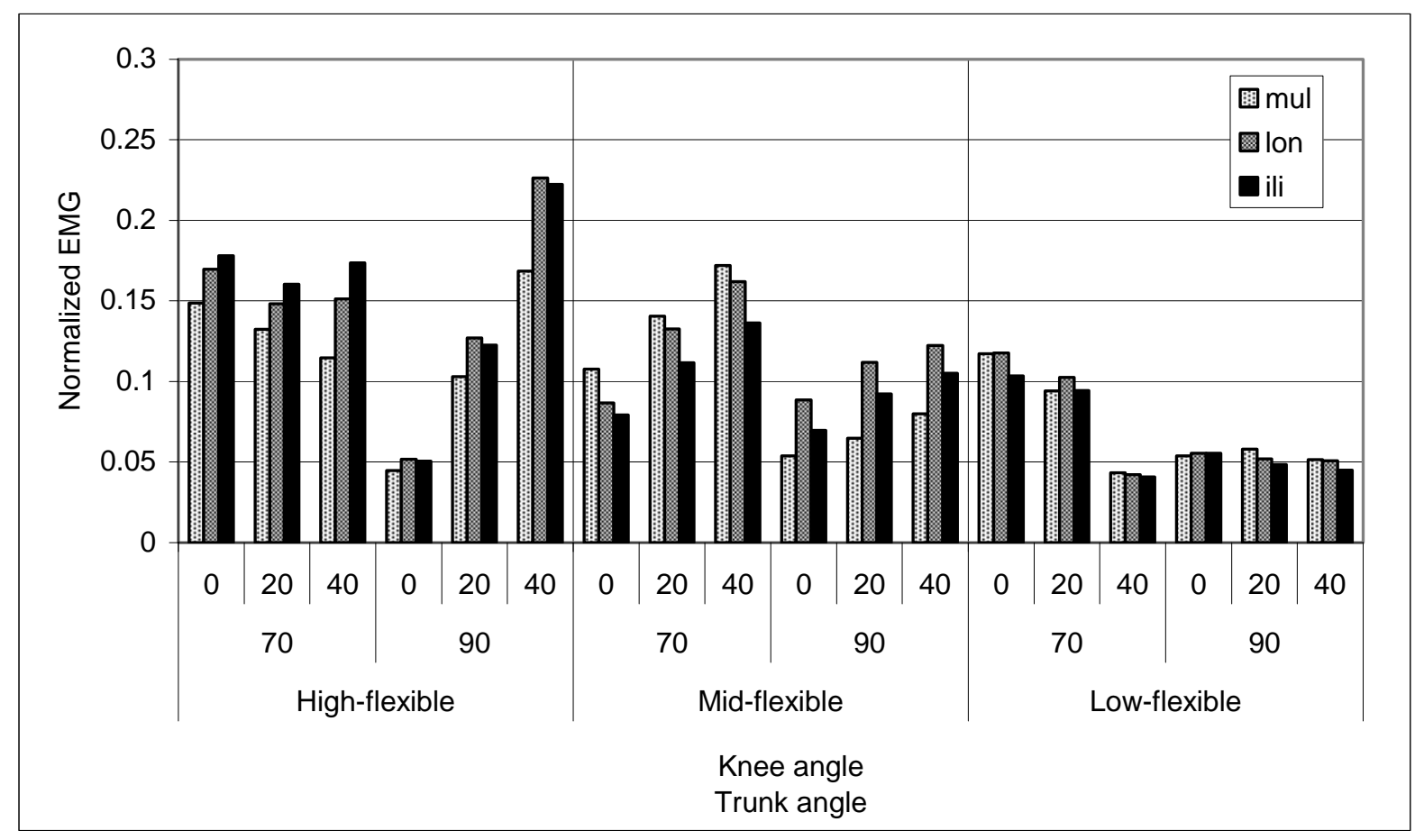

Figure 3.5. Normalized EMG of lumbar extensors vs. knee angle, trunk angle, and flexibility (50\% MVC condition) (mul : multifidus, lon : longissimus, ili : iliocostalis).

\subsection{Discussion}

In the 50\% MVC load condition, the external load on lumbar extensors was expected to be relatively consistent because the weight of the hand-held load varied to generate the same moments in change of flexion angle and moment arm. Different from this expectation, the normalized EMG of lumbar extensors decreased as a function of flexion angle from $30^{\circ}$ to $70^{\circ}$ trunk flexion angle, regardless of subjects' flexibility and knee flexion angle. This result confirms the idea that the passive tissues generate extension moment in weight holding and the amount of passively generated extension moment increases as the trunk flexion angle increases. 
In both load conditions, subjects' flexibility had significant effects on the EMG of all muscles. The trends in lumbar extensor muscle activity showed a consistent reduction across trunk angles with reduced flexibility. In this study a modified sit-and-reach test was used that had the subjects assume the same basic posture but in a standing position to gain a better simulation of the experimental task to be performed. The sit-and-reach test results have been found to be determined by hamstring flexibility (Simoneau, 1998). Since the length and tension on the hamstring have effects on the pelvic contribution to pelvis rotation (and thereby the passive spine extensor mechanism), relatively larger active extensor moments would be expected with high-flexible subjects than low or mid-flexible subjects and these were the findings of the current experiment. Further exploration of this flexibility response in the extreme flexion positions $\left(70^{\circ}\right.$ and $\left.90^{\circ}\right)$ showed that the knee angle response was significantly affected by the flexibility of the subject (Figure 3.5), leading to the formulation of a simple multi-joint articular model that considers these interactive effects.

While standing upright without knee flexion, the hamstring muscles and spine ligaments are in moderate length with little passive tension. Inclination of the torso from this position involves flexion of the vertebral column (including sacroiliac flexion) and hip flexion. The lumbar spine contributes more to early forward bending $\left(0-30^{\circ}\right)$ than the hip, and the hip contributes more to late forward bending $\left(60-90^{\circ}\right)$ than the lumbar spine (Esola et al., 1996). The pelvifemoral rhythm that describes these relative motions is expressed as the ratio of angle change between the pelvis and vertical reference to angle change between the femur and vertical reference. The contribution of pelvis rotation to hip flexion has been shown to vary as a function of knee angle. The mean ratio of pelvis rotation to hip flexion has been reported to be $26 \%$ when knees are flexed (Bohannon et al., 1985a), and 39\% when 
knees are straight, with subjects in the supine position (Bohannon et al., 1985b). In standing position, the pelvis rotation could contribute $18.1 \%$ to hip flexion (leg raise with knees flexed) (Murray et al., 2002). As the person bends forward from upright standing posture (Figure 3.6a), the pelvis freely rotates forward until the passive tension in the hamstrings begins to influence the pelvic rotation. As the person continues to flex forward (Figure 3.6b) and the forward rotation of the pelvis continues to be restrained, the passive extensor mechanism of the spine is activated. As this process continues with greater forward trunk flexion, the ratio of active to passive extensor moment continues to decrease to the point of full flexion-relaxation. When knee flexion occurs at this flexion-relaxation posture (Figure 3.6c), the distance between the origin and insertion of the biceps femoris is reduced and thereby releases the tension on the pelvis allowing it to rotate forward and deactivating the passive extensor mechanism requiring greater active moment from the lumbar extensors. Individual flexibility plays a role in this process in that the amount of knee flexion required to release hamstring tension is going to be dependent on this characteristic. In fact, in some combinations of trunk angle and flexibility no amount of knee flexion is going to be adequate to release the tension in the hamstrings and allow the pelvis to rotate forward (as seen in the low flexible group data in Figure 3.5). 


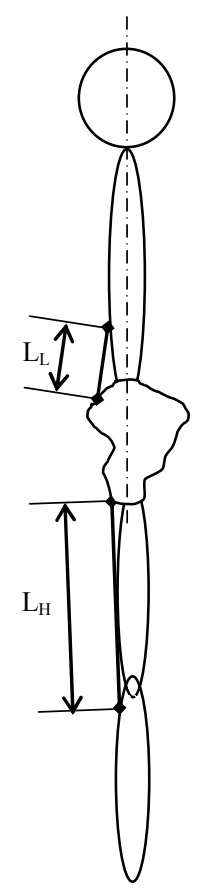

(a)

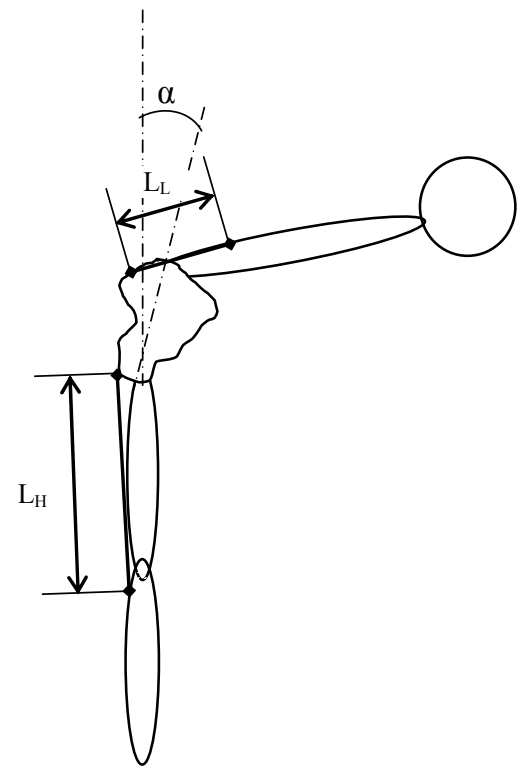

(b)

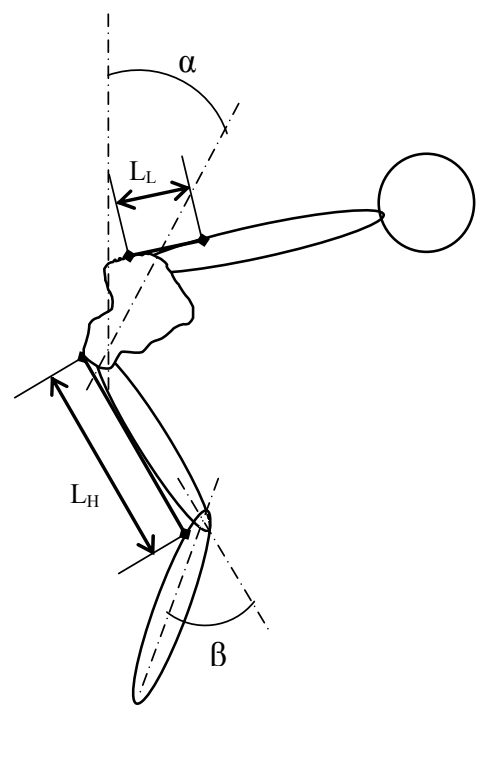

(c)

Figure 3.6. Simple biomechanical model showing the interaction between trunk flexion angle, knee flexion angle and individual flexibility. $H$ : distance between origin and insertion of hamstring; $L$ : length of a spine ligament. 


\section{VALIDATION OF CONCEPT AND VERIFICATION OF METHODS}

This section presents two small-sample $(n=1)$ preliminary experiments that were performed to demonstrate the ability to replicate previous empirical results as well as refine the experimental design/procedures of the dissertation research. Specifically, these experiments explored the main concept that prolonged stooping could generate viscoelastic responses of the lumbar spine such as changes in the range of lumbar flexion (Parts 1 and 2), changes in the muscle activity level of a static weight holding task (Part 1), and changes in the flexion-relaxation on/off angle during isokinetic flexion motions (Part 2). It was also verified that the experimental methods such as the data collection and the data analysis procedures could produce reliable results in the in vivo experiments to be conducted in the dissertation research (Chapter 6).

\subsection{Part 1}

\section{Objectives}

- To examine the change of the maximum lumbar flexion angle during and after stooping.

- To examine the effect of stooping period on the muscle exertion levels of static weight holding tasks at $30^{\circ}$ and $50^{\circ}$ flexion angles.

\section{Methods}

One human subject performed a prolonged (10-minute) stooped posture exertion which was followed by a period of recovery. Myoelectric activity (EMG) of lumbar extensors (multifidus and iliocostalis muscles at the L2 and L4 levels) was measured using surface electromyography and changes in lumbar angle in sagittal plane were captured using 
an electro-magnetic motion tracking system. A detailed description of these procedures/techniques is provided in Chapter 6 of this document.

The experiment started with maximum voluntary contraction (MVC) trunk extension exertions at $30^{\circ}$ and $50^{\circ}$ of trunk flexion in a lumbar dynamometer (Figure 4.1). After completion of the MVC exertions, the subject was secured to a fixture designed to secure/stabilize the pelvis. The fixture had two padded arms that pushed against the pelvis backward against a fixed board so that the trunk flexion due to pelvis rotation was minimized. Both knees were also locked by a padded strap around the upper leg area (Figure 4.2).

Data collection consisted of two phases: a stooping phase and a recovery phase. Before the stooping phase, the subject performed static weight holding tasks at $30^{\circ}$ and $50^{\circ}$ trunk flexion with a $9 \mathrm{~kg}$ load while the extensor muscle activities were collected for 3 seconds. After a 2-minute rest break, the subject flexed his back slowly and reached a full flexion posture. The full flexion posture was described as a slumped posture with no muscle activities, and upper body weight was supported passively. When the full flexion posture was achieved, the subject was asked to flex more using his abdominal muscles to reach maximum lumbar flexion angle, maintain the posture for 5 seconds, and return to the full, relaxed flexion posture. The stooping phase started as soon as the subject returned to the full flexion posture. During the 10-minute stooping period, the subject maintained the full flexion posture and performed the maximum lumbar flexion (maximal exertion of the abdominal muscles) every 2 minutes $\left(2,4,6\right.$, and $8^{\text {th }}$ minute), and weight holding $(9 \mathrm{~kg})$ tasks at $30^{\circ}$ and $50^{\circ}$ flexion angle every 5 minutes ( $5^{\text {th }}$ and $10^{\text {th }}$ minute).

At the end of the weight holding tasks at the $10^{\text {th }}$ minute, the subject stood up and the recovery phase began. The recovery phase continued for 7 minutes with static weight 
holding tasks every odd minute $\left(1,3,5\right.$, and $7^{\text {th }}$ minute). The muscle activities of the extensor muscles were captured during these weight holding exertions.

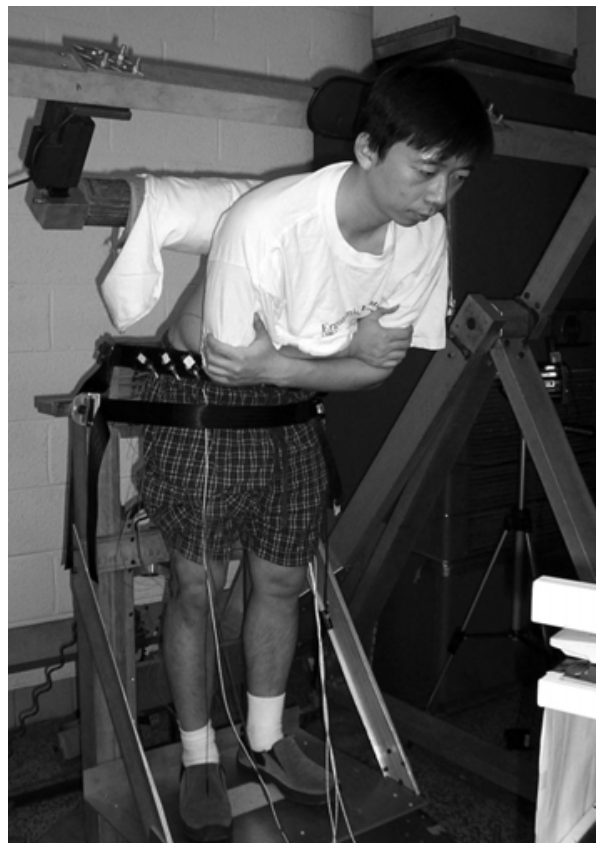

Figure 4.1. MVC exertion in a lumbar dynamometer.

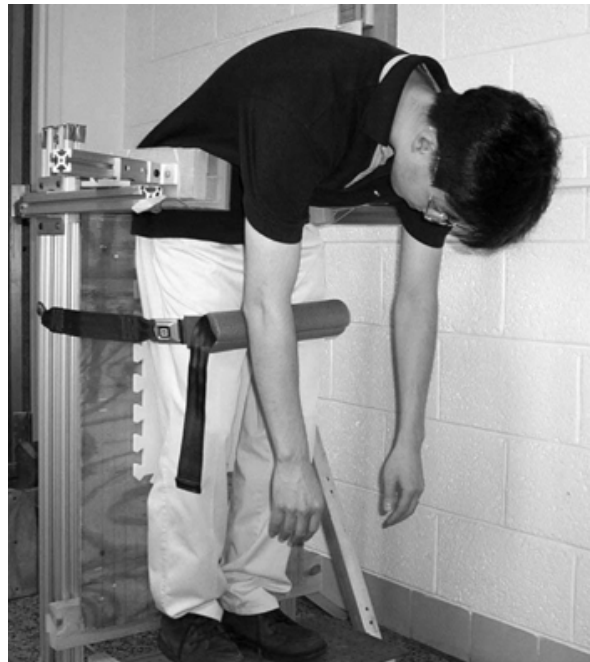

Figure 4.2. Full flexion posture in a fixture. 


\section{$\underline{\text { Results }}$}

The maximum lumbar flexion angle before stooping was observed as $49.4^{\circ}$ and it showed consistent increase to $51.3^{\circ}(3.8 \%$ increase) in stooping period (Figure 4.3$)$. This increase in the maximum lumbar flexion angle, while the pelvis was secured on the fixture, indicated an increase of range of motion (in sagittal plane) of the lumbar spine structure due to elongation of posterior ligaments or deformation of the intervertebral discs under creep loading condition.

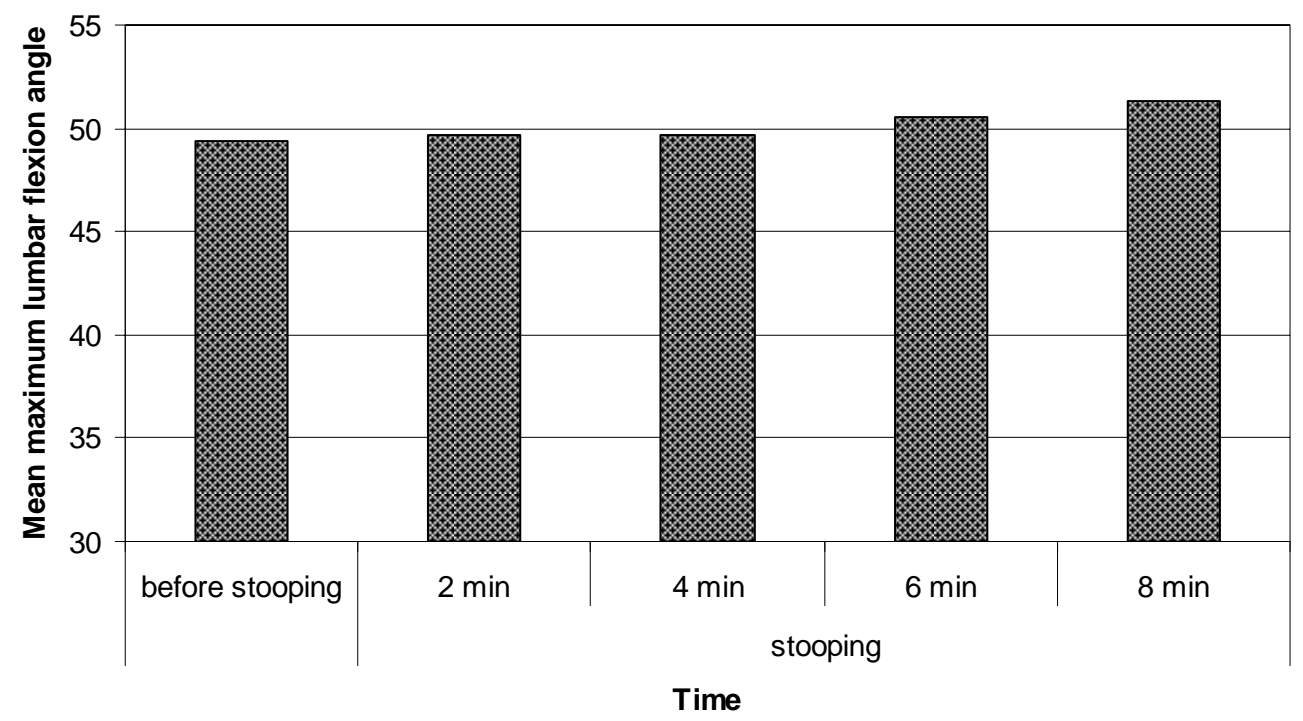

Figure 4.3. Mean maximum lumbar flexion angle during stooping period.

Normalized EMG collected during the weight holding tasks are shown in Figures 4.4 and 4.5. Normalized EMG values in stooping $\left(5^{\text {th }}\right.$ and $10^{\text {th }}$ minute) were greater than the EMG before stooping and in recovery phase, except L4 level multifidus muscle EMG at $30^{\circ}$ trunk flexion. 


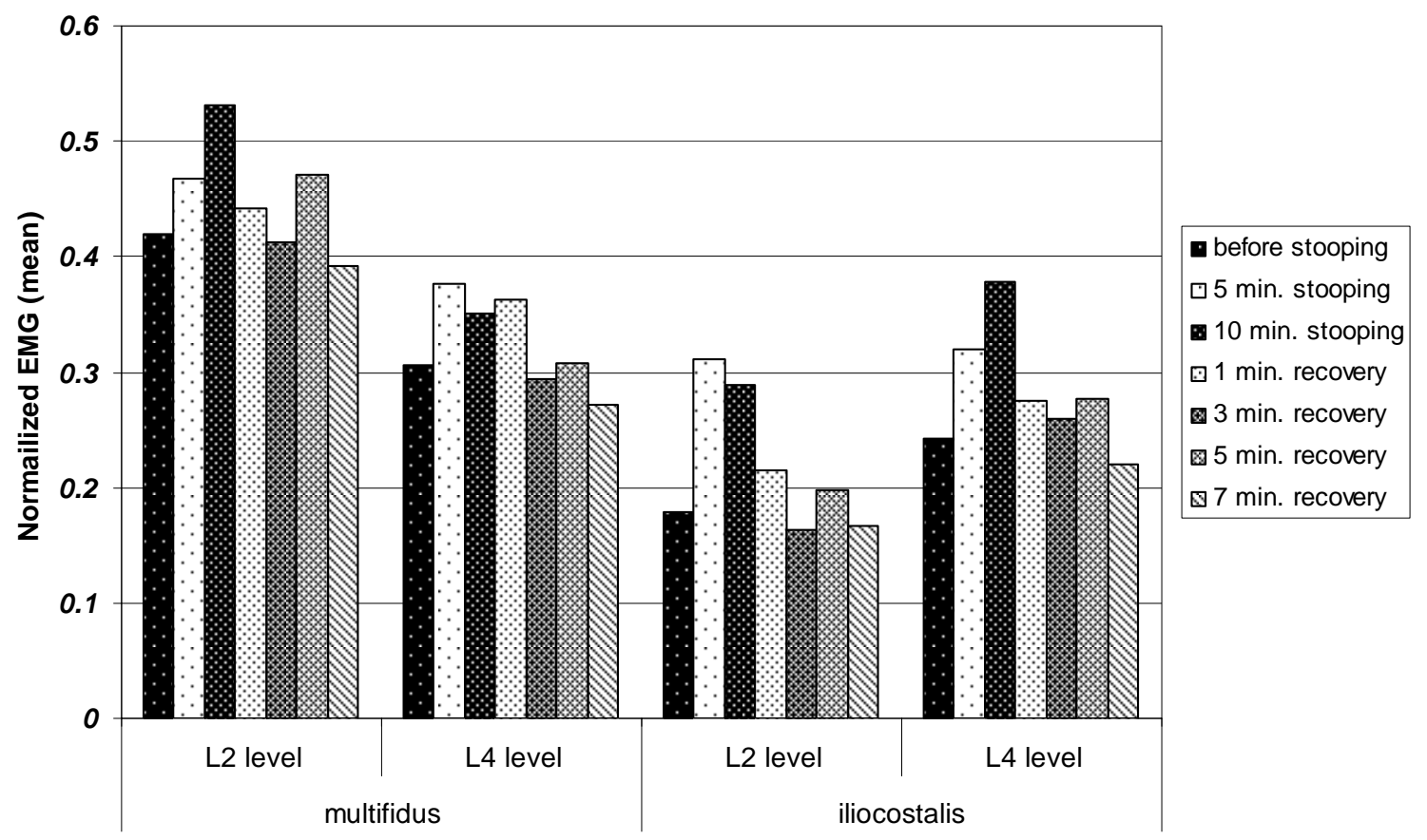

Figure 4.4. Normalized EMG collected at $30^{\circ}$ of trunk flexion during the weight holding tasks.

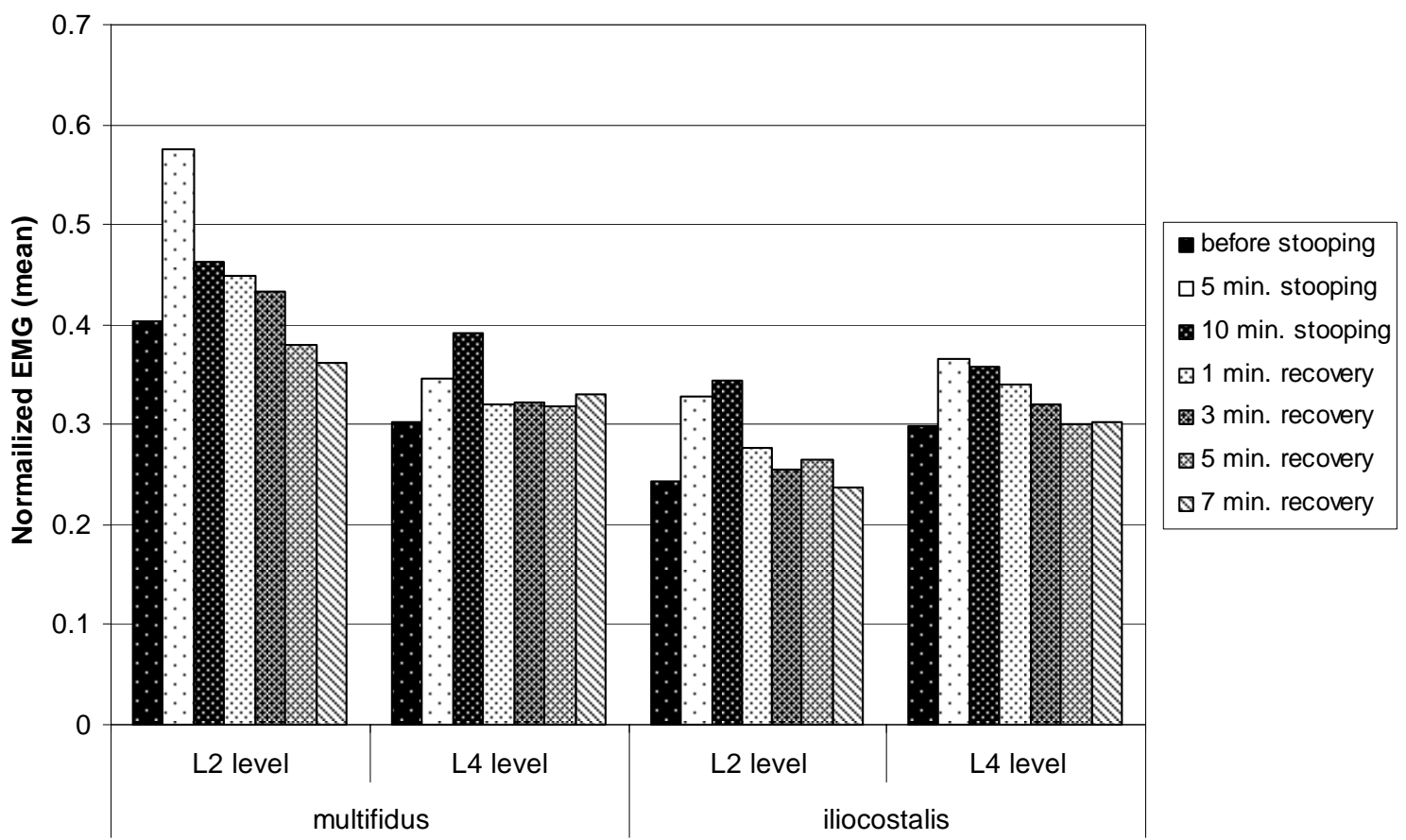

Figure 4.5. Normalized EMG collected at $50^{\circ}$ of trunk flexion during the weight holding tasks. 


\subsection{Part 2}

\section{Objectives}

- To examine the change of the relaxed, full lumbar flexion angle during and after stooping.

- To examine the changes in the onset angle of flexion-relaxation in slow-speed, isokinetic flexion motions during stooping and recovery.

\section{Methods}

In this protocol, the myoelectric activities of extensor muscles (multifidus and iliocostalis at L2/L3 level) and lumbar flexion angle were collected with the same preparations and setup as used in the Part 1.

The protocol began with an MVC exertion at $60^{\circ}$ flexion angle in the lumbar dynamometer. The subject was then secured into the pelvis restraining fixture. Data collection consisted of a 12-minute stooping and a 10-minute recovery phase. Before the stooping phase, the subject performed two repetitions of sagittally symmetric isokinetic trunk flexion from $20^{\circ}$ flexion angle to the full lumbar flexion posture, with a $5 \mathrm{~kg}$ weight in hands. The time to finish lowering (flexion motion) was set to 20 seconds to minimize motion artifacts of EMG. During the flexion motion, the EMG and lumbar flexion angle were collected simultaneously to identify the onset angle of the flexion-relaxation and full lumbar flexion angle. The subject maintained the full flexion posture for 5 seconds and returned to upright standing posture.

After a 2-minute rest break, the subject flexed his back again to the full flexion posture and the stooping phase began. The static full flexion posture was maintained for 12 
minutes. Interspersed in this 12-minute period were the isokinetic flexion tasks every odd minute $\left(1,3,5\right.$, and $7^{\text {th }}$ minute).

The recovery phase started immediately after the 12-minute stooping phase was finished. The subject was asked to stand up slowly and keep a neutral standing posture with no load on hands. The same isokinetic flexion task was carried out every odd minute, and returned to the neutral standing posture after each isokinetic flexion task. The recovery period continued for 10 minutes.

The same data analysis methods, which were used to identify the full flexion angle in Part 1, were used in this protocol. To quantify the flexion-relaxation onset angle, the EMG data collected during 20-second isokinetic flexion motions were divided into 20 sections (one section per second) and the EMG data in each section was averaged to identify an average muscle activity within this one-second interval. When the normalized EMG went below 10\% of MVC EMG, the lumbar flexion angle at that instant was identified as the onset angle of flexion-relaxation.

\section{$\underline{\text { Results }}$}

Changes in the mean full lumbar flexion angle as a function of time are shown in Figure 4.6. The angle increased gradually during the stooping period and then decreased during the recovery period. The largest mean full flexion angle was observed at $9^{\text {th }}$ minute of stooping period and it was $20 \%$ greater than the mean full flexion angle before stooping.

The flexion-relaxation onset angle results are shown in Figure 4.7. The flexionrelaxation (10\% of MVC EMG) occurred at $45.4^{\circ}$ (average of two muscles) before stooping, but it did not occur until the subject flexed greater than $51.7^{\circ}$ after stooping period started. 


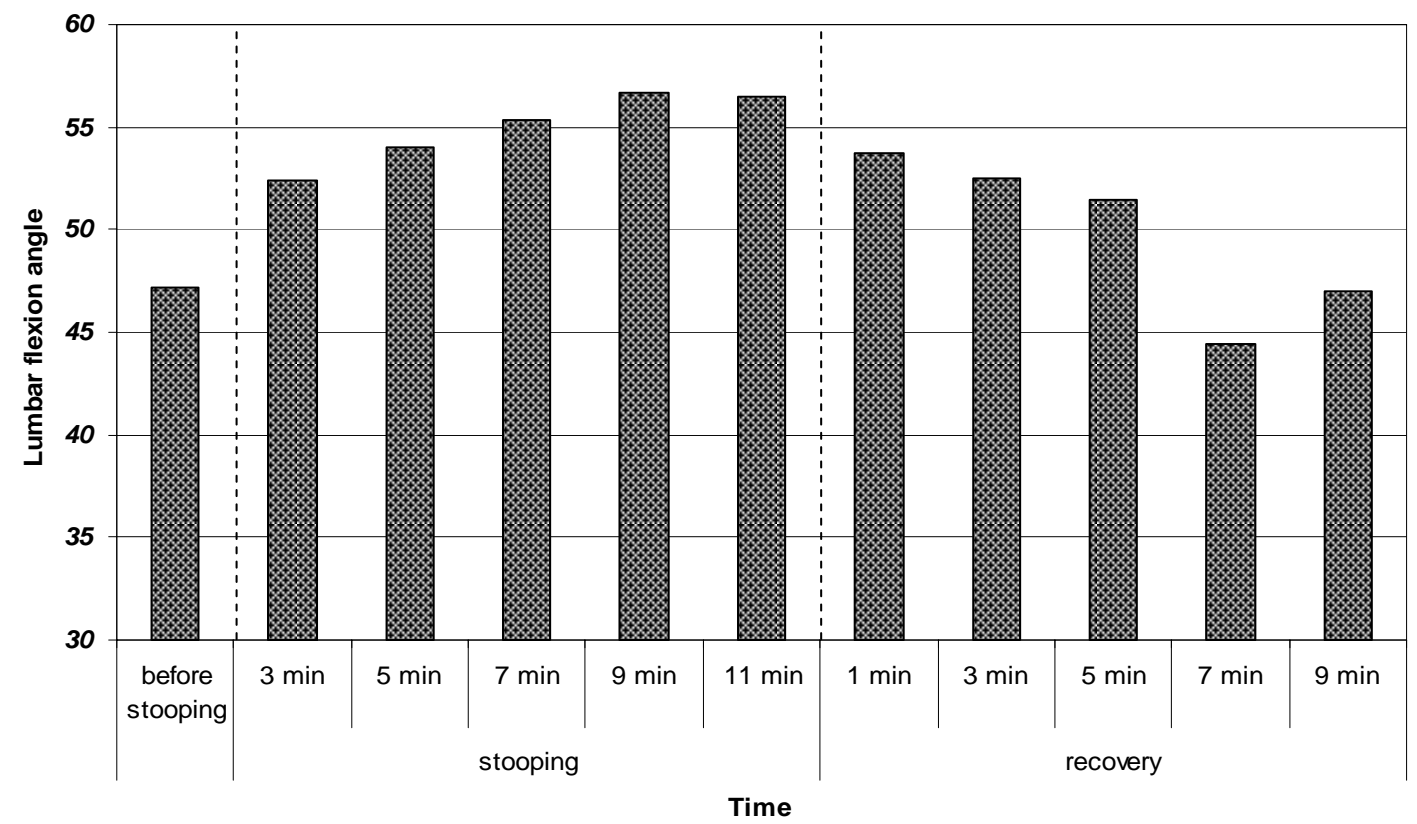

Figure 4.6. Mean full lumbar flexion angle.

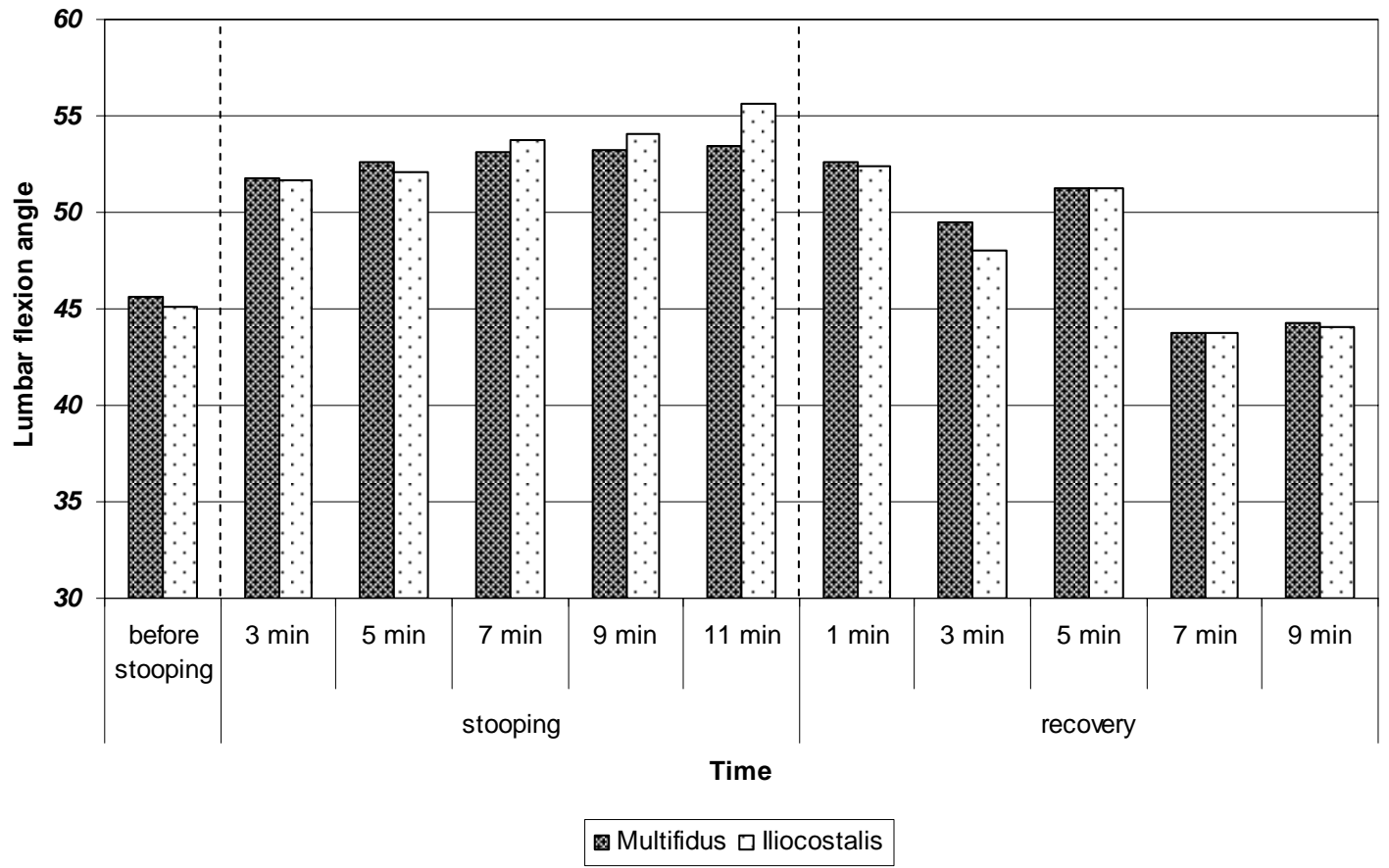

Figure 4.7. Flexion-relaxation onset angle (10\% max EMG). 


\subsection{Discussion}

Both verification studies were carried out by the same subject (male, 26 years old) with a 2-week interval between them to allow full recovery of the passive tissues in the lumbar spine. In the first experiment, the maximum lumbar flexion angle increased from $49.4^{\circ}$ at the beginning to $51.3^{\circ}$ after 8 minute stooping, which was a $3.8 \%$ increase. This amount of angle change was smaller than McGill and Brown's (1992) observation (6.1\% for a 8-minute full flexion) but greater than Solomonow et al. (2003a) result $(0.5 \%$ for a 10 minute full flexion). This could result from methodological differences among the studies. McGill and Brown (1992) measured full lumbar flexion angles using magnetic sensors on S1 and T12/L1 while their subjects were sitting fully flexed with pelvis clamped, and Solomonow et al. (2003a) used a video-based motion analysis system and measured the angle when subjects were sitting on a mat with a hemicylindrical foam bolster under the hips to tilt the pelvis posteriorly. Even though the lumbar flexion angle in vivo is somewhat variable depending on the measurement methods and subject's flexibility, the maximum flexion angle that observed in the first experiment $\left(49.4^{\circ}-51.3^{\circ}\right)$ was consistently smaller than other

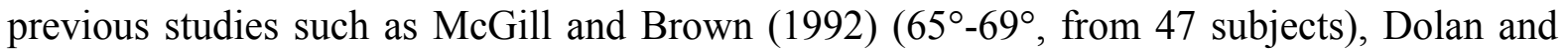
Adams (1993) $\left(56.4^{\circ}\right.$ range of motion, from 10 subjects), Dickey et al. (2003) $\left(58^{\circ}-60^{\circ}\right.$, from 30 subjects), and Solomonow et al. (2003a) $\left(59.8^{\circ}-60.1^{\circ}\right.$, from 49 subjects). Based on the feedback from the subject, it was thought that the clamps of pelvis restraint frame were pushing the upper area of the pelvis so the flexion of the lower lumbar region might be restrained and the range of motion of lumbar flexion could be reduced. In spite of this limitation, the results suggested that the procedures and setup for this validation work were 
capable of generating viscoelastic strain of lumbar spine tissues and detecting creep responses under prolonged static flexion.

The muscle activities measured at $30^{\circ}$ and $50^{\circ}$ trunk flexion angle throughout the experiment showed an effect of stooping on the level of EMG. Greater muscle activity level during the stooping period indicated that muscles had to work harder to support the extension moment, which was assumed to be constant. The increase of the muscle activity under the same external moment could be explained as a result of ligament strain during stooping period. As observed in our previous research (Shin et al., 2004) the tension of passive tissues, specifically posterior ligaments, have influence on the muscle exertion level during weight holding tasks at lower angles than flexion-relaxation angle. Spinal posterior ligaments were stretched during the prolonged stooping task and the creep of the ligaments over time could reduce the tension in the ligaments, generating similar effect to reduced tension due to knee flexion. Since the posture was supposed to be the same for each flexion angle of the weight holding task, the extensor muscles should contribute more to generating extension moment to compensate for the loss of extension moment due to reduced tension of ligaments. After 5 minutes of the stooping period, the EMG collected during the static weight holding tasks were $29.8 \%$ and $29.1 \%$ greater than the EMG of pre-stoop phase of $30^{\circ}$ and $50^{\circ}$ trunk flexion, respectively. The increase of the EMG during the static weight holding was seen also after repeated spinal flexion by Dickey et al. (2003). Their subjects repeated 100 full flexions for 18 minutes of period and the EMG increased more than 50\% compared with pre-lift phase. They suggested that the creep of passive tissues might cause the change of the EMG and the flexion-relaxation angle after repetitive lifts. 
To resolve these limitations of the first experiment, the second experiment (Part 2) used a modified pelvis restraint system and a different approach for assessing creep responses. The maximum lumbar flexion trials during static stooping posture in the first experiment (Part 1) might cause micro damage on passive tissues and the angle could be affected by the fatigue or exertion levels of abdominal muscles. For subject safety, and to minimize uncontrollable variables, Experiment 2 investigated the lumbar flexion angle in a relaxed stooping posture (full lumbar flexion angle), which did not require any muscle exertions (including abdominal muscles), instead of the maximum lumbar flexion angle. The full lumbar flexion angle was collected when the subject reached a stable stooping posture at the end of the isokinetic flexion. Results in Figure 4.6 confirmed the hypothesis that the full lumbar flexion angle would increase as a function of stooping period and then reduce as a function of recovery period, suggesting the flexion angle at relaxed stooping would be more efficient and reliable to see the creep responses than the maximum lumbar flexion angle.

The onset angle of flexion-relaxation was identified in Experiment 2 by looking at the lumbar flexion angle when the normalized EMG reached 10\% max. Results indicated that the flexion-relaxation occurred at greater lumbar flexion angle as the static stooping time increased and the recovery in a neutral upright standing posture made the flexion-relaxation angle smaller toward the original angle (before stooping period). The increase of flexionrelaxation onset angle after the creep was also observed in previous in vivo studies. Dickey et al. (2003) found an increase of the angle from about $93 \%$ of max angle to $95 \%$ max angle after 100 repetitive lifts, and Solomonow et al. (2003a) also observed a 9.5\% increase from $46.1^{\circ}$ to $50.5^{\circ}$ after a 10 -minute static lumbar flexion. Although their results are not comparable with the current preliminary work because of methodological differences and 
inter-subject variations, the results of this work suggest that the creep occurred in the lumbar spine structure during static stooping and recovery period and it affected the onset angle of flexion-relaxation.

The end result of these two small exploratory protocols was a confidence in our ability to create a measurable viscoelastic response in a controlled experimental environment. With this procedure in place we were able to implement experimental manipulations to further explore this response. 


\section{GENERAL OBJECTIVES AND STRUCTURE OF THE STUDY}

The main objectives of this study were to quantify the viscoelastic responses of the lumbar spine due to prolonged stooping and develop a finite element (FE) model that can be used as a tool to predict these responses. This model could then be considered as a precursor for a risk assessment tool able to consider variables (duration, rest-break profiles, loading, etc.) describing prolonged stooping tasks.

This study consists of both an in vivo experiment and FE model simulations. The in vivo human experimentation is performed to gather empirical data describing creep deformation and stress-relaxation of lumbar spine passive tissues on muscle activity level. Second, the FEA study proposes the rationale of developing a new tool to assess risks of prolonged stooping using the FEA technique, leading to the creation of a nonlinear viscoelastic 3-D FE model of the lumbar. Finally, the empirical data collected in the in vivo experiment are simulated by the FE model to validate the model by comparing the predicted results with the experimental observations. 


\section{IN VIVO EVALUATION}

\subsection{Introduction}

Low back pain (LBP)/ disorders (LBDs) represent a significant category of workrelated nonfatal injuries. Data provided by the Bureau of Labor Statistics (BLS) shows that cases involving back injuries were reported to be over 345,000 in 2002 (Bureau of labor statistics, 2004). LBDs are a common disorder and affect up to $85 \%$ of the population sometime during their lifetime (Korff et al., 1988; Andersson et al., 1998). Occupational LBDs can cause large economic losses such as workers' compensation costs and lost earnings from lost work days. In a study of workers' compensation claims of Liberty Mutual Insurance company during 1989 , the LBP cases accounted for $16 \%$ of the total number of all compensation cases, and the total cost for all LBP cases accounted for $33 \%$ of the total cost of all compensable claims. Generalizing these results to other insurance companies, it was estimated that the total worker's compensation costs for LBP cases that occurred in the United States in 1989 was \$11.4 billion (Webster and Snook, 1994). Guo et al. (1999) analyzed the 1988 National Health Interview Survey to estimate the number of lost work days due to work-related back pain. The total number of lost work days as a result of workrelated back pain was estimated to be 101.8 million days in United States. Using the median weekly wage (\$467) in 1995, the lost earning from lost work days due to work-related back pain was estimated to be approximately $\$ 9.5$ billion.

LBDs can result from one or a combination of physical risk factors such as static awkward postures (Punnet et al., 1991; McGill, 1997), frequent bending and twisting (Marras et al., 1995), lifting and forceful movement (Waters et al., 1999), and vibration (Pope et al., 1989). Static awkward postures can include extreme sagittal trunk bending (stooped posture), 
lateral bending, and transverse rotation. These awkward postures are often observed to continue for a prolonged period of time in many work environments.

There are a number of industries that have work tasks that require the worker to work at or below knee height for extended periods of time with little or no lifting/lowering of the torso. This prolonged stooped posture has been considered as a key element that can cause LBDs (McGill, 1997). Many tasks in the agriculture and construction industries often require these postures and these industries note high levels of low back injury (e.g. agricultural production crop (Incidence Rate (IR) of back pain $=5.14$ cases per 100 full-time workers), landscape and horticultural service (IR of back pain $=5.92$ ), roofing work (IR of back pain $=5.95)$ and concrete work in construction site $(\mathrm{IR}$ of back pain $=4.56))$. These jobs require substantial time spent in stooped postures and those tasks reported higher incidence rates of LBD compared to average industry (Bureau of Labor Statistics Report, 2004).

In the stooped posture, an evaluation of the biomechanics of the lumbar spine must consider the responses of passive tissues and their effects on the lumbar spine stability and muscle exertion level. When the torso is flexed forward from the upright standing posture, the back extensor muscles generate eccentric contraction force and provide extension moment supporting the mass of upper body. At the same time, posterior ligaments and other passive tissues are strained and gradually generate extension moment by passive resistant forces as they are elongated. As the flexion angle increases the passively generated moment from these tissues takes over from active contraction force of the back extensor muscles as the main contributor to supporting the body weight and finally the extensor muscles exhibit myoelectic silence as the torso reaches the fully flexed posture (Floyd and Silver, 1955; 
Kippers and Parker, 1984; Schultz et al., 1985; McGill and Kippers, 1994; Shin et al., 2004).

The contribution of the passive tissues to generating extension moment has also been observed during lifting motions. Dolan et al. (1994) assessed the passive extensor moment during lifting. Their subjects performed isometric upward pull tests with steadily increasing force on a floor-mounted load cell while EMG in erector spinae muscles were recorded. The relationship between the EMG and the extensor moment (calculated from the load cell data) was linear with the intercept (at ' 0 ' EMG) on the moment axis which indicated the portion of the moment resisted by passive tissues. They computed that between 16 and $31 \%$ of the peak extensor moment during the pull test was unrelated to the EMG activity in the erector spinae, and less than a quarter of this passively generated extensor moment was come from the intervertebral discs and ligaments. If the fully flexed or stooped posture is maintained, the passive tissues continue to deform at a slow rate as a function of time because of their viscoelastic material properties (Burns et al., 1984; Li et al., 1995; Nachemson and Evans, 1968).

The time-dependent responses of viscoelastic materials can be described by creep deformation under conditions with a constant load and stress-relaxation under conditions with a constant strain. The creep deformation of the human lumbar spine has been observed in vivo in terms of a stature decrease after a long period of compressive load (Van Dieën et al., 1994), increase of flexion-relaxation on/off angle after prolonged static full flexion (Solomonow et al., 2003a) and after 100 consecutive full trunk flexion and return to standing maneuvers (11 seconds per maneuver) (Dickey et al., 2003), and the change of full lumbar flexion angle during prolonged stooped posture and recovery period in sitting (McGill and Brown, 1992). Stature decrease, after holding a weight for a prolonged period, might be 
related to the creep deformation of the intervertebral disc (Keller et al., 1990; Burn et al., 1984; Adams and Dolan, 1996) under a compression load on the spine. Greater flexionrelaxation on/off angle after prolonged stooping or repetitive lifting implied the creep deformation of passive tissues, specifically on the posterior side of the lumbar spine (Adams and Dolan, 1996). The time-dependent changes in the full lumbar flexion angle of in vivo human subjects during 20-minute static full lumbar flexion and following 30-minute recovery was examined by McGill and Brown (1992). The full lumbar flexion angle increased by $5.5^{\circ}$ over 20 minutes and decreased toward the initial full angle during recovery, but was not fully recovered in 30 minutes. This study showed the viscoelastic responses of the lumbar spine during a recovery period as well as the prolonged stooping period.

The creep deformation and the stress-relaxation of intervertebral discs and other passive tissues provide more laxity in the spine and reduced resistance to forward flexion moment (Adams and Dolan, 1996; Solomonow, 2004). These mechanical changes are known to allow excessive motion in the lumbar spine (Adams et al., 1987), impair spinal stability (Cholewicki and McGill, 1996), and may require greater level of muscle force to compensate for the loss of passively generated forces (Adams and Dolan, 1996). It is reasoned that these mechanical changes may lead to an increased risk of low back injury. The decrease of stability in the lumbar spine has been known to affect the intervertebral disc pressure and the muscular activity level and finally result in pain or disorders in low back (Crisco et al., 1992; Solomonow, 2004). Studies investigating the relationship between spinal instability and LBD have reported the abnormal segmental motion as a cause of degeneration and/or LBD (Nachemson, 1985). 
In addition to mechanical viscoelastic properties, neurological properties of spinal ligaments could induce disorders in the low back. Spinal ligaments contain mechanoreceptors and have been shown to elicit a reflexive activation (spasms) and inhibition of muscles (Solomonow et al., 2003b). Solomonow et al. (2003b) suggested that static loading of the lumbar spine ligaments could cause micro-damage in the tissues and it further resulted in eliciting spasms and a gradually decreasing reflexive muscle activity in static loading periods. The micro-damage can produce the time-dependent development of acute inflammation in the ligaments and it renders increased reflexive muscular forces in order to protect the damaged tissues and help the healing process during the rest period.

Despite the greater demand on the muscle activity during and after the prolonged stooping, the extensor muscles during and after stooping may not be as functional as the muscles before stooping. Muscle-tendon units respond viscoelastically to passive stretching. Continuous or repeated passive stretch of muscle-tendon units leads to reduced passive tension force and increased length (Taylor et al., 1990; Magnusson, 1998; McHugh et al., 1992), and these mechanical changes may have influences on the motor unit activation (Avela et al., 2004). Muscle-tendon units have shown reduced motor unit activation and reduced force-generating capacity after passive stretch and gradual recovery was observed during rest period (Fowles et al., 2000; Avela et al., 2004). In a prolonged stooped posture, the back extensors generate substantial extension moment by passive stretching of muscletendon units (McGill and Kippers, 1994) and this may cause reduced strength performance of the back extensor muscles.

Simultaneously, micro-damage in the spinal ligaments and the passive stretch of muscle-tendon units can degrade the force-generating capacity of extensor muscles. Greater 
demand on weaker muscles can result in faster muscle fatigue generation and/or failure in the muscles' ability to maintain lumbar stability. This is particularly critical when the lumbar spine is subjected to a high external load immediately after prolonged stooping, as is often observed in many occupational environments (e.g. construction, agriculture).

Recovery of the normal (i.e. unaltered from resting state) function of the viscoelastic tissues begins when the load is removed but full recovery may require more time than the duration of the activity that created the alteration in these responses. The creep deformation developed during 20 minutes in a stooped posture was not fully recovered in a 30-minute resting posture (McGill and Brown, 1992), and an in vivo feline model exhibited a large cumulative (i.e. residual) creep of a spinal ligament after repetitions of 10-minute static flexion followed by 10-minute rest (Solomonow et al., 2003b). A feline model also showed that creep in ligament during 20-minute static flexion did not fully recover over a 7-hour rest period, and suggested the full recovery from 20-50 minutes of static flexion may require 2448 hours of rest (Solomonow et al., 2003b). Repetitive stooping tasks without enough rest can produce cumulative creep in the lumbar spine and inflammation in the ligament, and finally make the lumbar spine vulnerable to instant or chronic musculoskeletal disorders (Figure 6.1). 


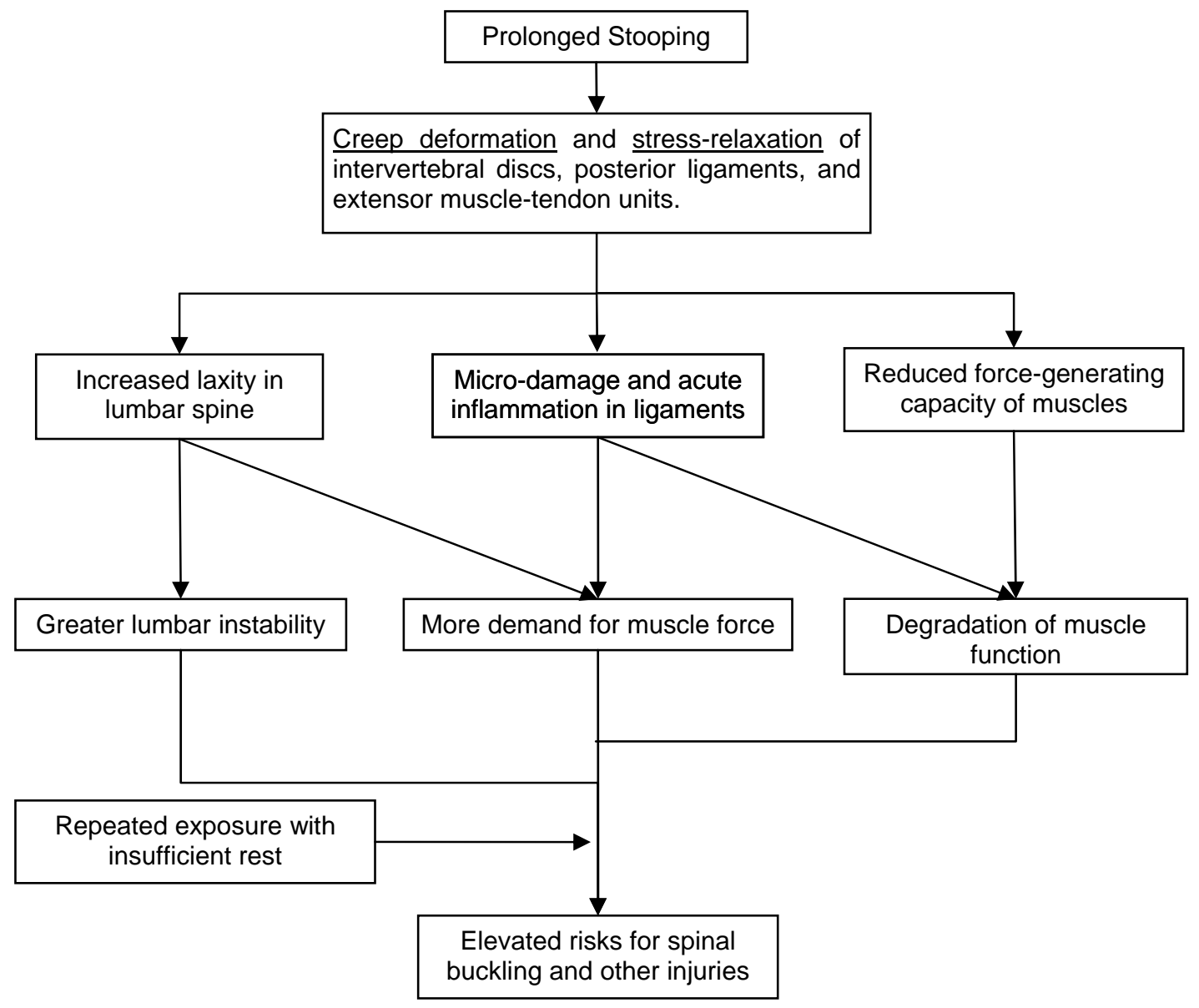

Figure 6.1. A conceptual model relating the risk factors of prolonged stooping.

Muscles have a major role in maintaining lumbar stability and protecting damaged tissues in prolonged stooping and recovery. Understanding how the actively generated force of the back extensor muscles changes over a prolonged stooping and recovery period and how it relates to the creep deformation of the lumbar spine is important to assess the risks of prolonged stooping.

Since the responses of the lumbar spine in prolonged stooping are time-dependent and a substantial amount of mechanical recovery of passive tissues occur mostly at the beginning of the recovery period (Solomonow et al., 2003b; McGill and Brown, 1992), a rest break in 
the middle of stooping may reduce the level of cumulative creep deformation and/or can influence the muscle activity level. Frequent micro or short breaks in prolonged stooping can be observed in many occupational tasks but no in vivo human study has been performed to assess the effects of a micro-break in the middle of stooping on the viscoelastic responses of the lumbar spine.

The first objective of this study was to examine the time-dependent changes in the back extensor musculature to evaluate the effects of the creep deformation of the lumbar spine during and after the prolonged stooping and to see the transfer of the extension moment between passive tissues and active muscles. Second objective was to evaluate the effects of a short "micro-break" recovery period during a prolonged stooped posture. These effects will be evaluated in terms of the time-dependent changes in the full lumbar flexion angle as well as the muscle activity during prolonged stooping and recovery.

\subsection{Methods}

\subsubsection{Subjects}

Five male and five female subjects (mean age: 27.8 years, SD of age: 2.7 ) without current or chronic low back pain/injury were recruited from the university community. All subjects signed an informed consent form prior to participation in the experiment. Since the physical properties of passive tissues vary with age, subjects under 20 and over 35 years old age were not recruited to minimize variation due to age. Anthropometric characteristics of the participants are shown in Table 6.1 . 
Table 6.1. Subjects' anthropometric data.

\begin{tabular}{cccc}
\hline & Age (years) & Weight $(\mathrm{kg})$ & Stature $(\mathrm{m})$ \\
\hline Male & & & \\
1 & 32 & 78.0 & 1.767 \\
2 & 29 & 60.8 & 1.733 \\
3 & 26 & 67.1 & 1.800 \\
4 & 30 & 59.9 & 1.737 \\
5 & 25 & 73.5 & 1.799 \\
Mean (SD) & $28.4(2.89)$ & $67.9(10.6)$ & $1.767(0.0323)$ \\
Female & & & \\
1 & 24 & 56.2 & 1.656 \\
2 & 27 & 54.0 & 1.575 \\
3 & 30 & 50.8 & 1.669 \\
4 & 25 & 80.3 & 1.657 \\
5 & 30 & 54.9 & 1.709 \\
Mean (SD) & $27.2(2.78)$ & $59.2(11.9)$ & $1.653(0.0487)$ \\
\hline Overall Mean (SD) & $27.8(2.74)$ & $63.5(10.6)$ & $1.710(0.0716)$ \\
\hline
\end{tabular}

\subsubsection{Apparatus}

Myoelectric activity of lumbar extensors was measured using four pairs of $\mathrm{Ag}-\mathrm{AgCl}$ surface electrodes. The electrodes for the erector spinae muscles were secured bilaterally on the skin $5 \mathrm{~cm}$ lateral to the midline of the spine at the L3 level. The electrodes for the multifidus muscles were secured bilaterally on the skin $3 \mathrm{~cm}$ lateral to the midline of the spine at the L4 level (Figure 6.2). A lumbar dynamometer (Asymmetric Reference Frame (Figure 6.3)) was used to provide necessary static resistance for the collection of anglespecific peak moment generated by subjects. These data were necessary for the calculation of hand-held loads to be used in the subsequent experimental trials. EMG data collected during maximum voluntary contractions (MVCs) in this apparatus were also used for normalization of the experimental EMG data. The EMG signals were recorded at $1024 \mathrm{~Hz}$. 
An electro-magnetic motion tracking system (Motion Star, Ascension Technology Corp. Burlington, VT.) measured the lumbar flexion angle. The relative angle between two magnetic sensors that were located on the skin surface overlying the spinous processes of T12/L1 and L5/S1 was defined as the lumbar flexion angle. The system has a $0.1^{\circ}$ RMS static angular resolution, and this resolution was verified in the current experimental environment throughout all angles ranging from $-10^{\circ}$ to $60^{\circ}$ (between two sensors) for static measurements. The data from the two electro-magnetic sensors were recorded at $42.66 \mathrm{~Hz}$. This sampling frequency was specifically selected because the electrical noise from the transmitter of this system could influence the EMG data and this noise can be easily eliminated by filtering software if the frequency is 1/12 or 1/24 of $1024 \mathrm{~Hz}$ (EMG sampling frequency). Between the $85.33 \mathrm{~Hz}$ and $42.66 \mathrm{~Hz}$ frequencies, the lower one $(42.66 \mathrm{~Hz})$ was chosen to minimize metal interference error between an aluminum fixture and the motion tracking system.

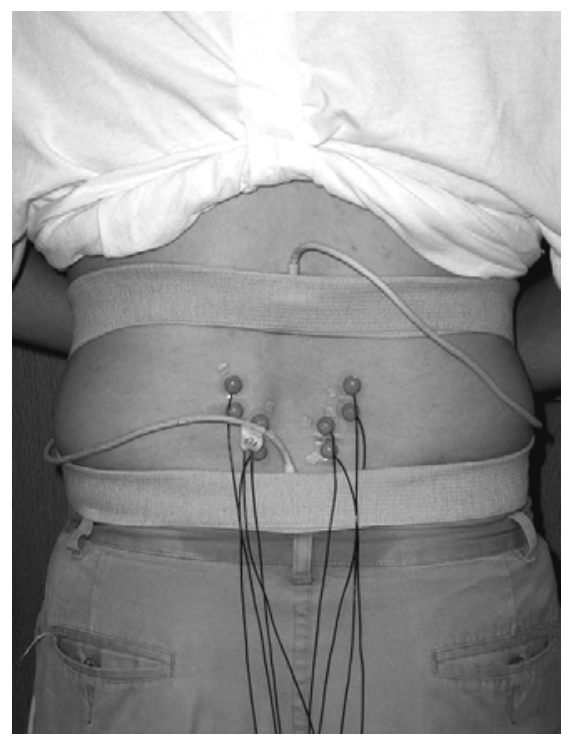

Figure 6.2. Surface electrodes and electro-magnetic sensors.. 


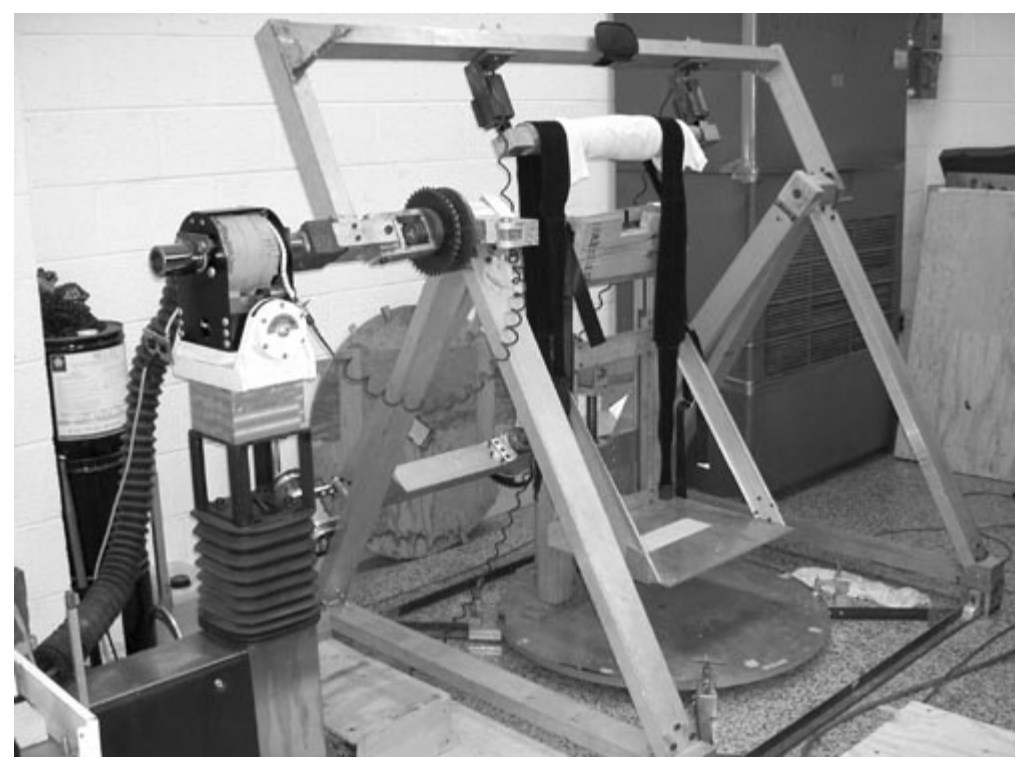

Figure 6.3. Asymmetric reference frame.

\subsubsection{Experimental Procedures}

This experiment spanned two days of data collection: one day without a rest break (no-rest condition) and the other day with a rest break (w/rest condition). The order of the no-rest day and the w/rest day was randomly assigned to subjects. The two experimental sessions were separated by a one-week interval to allow enough recovery of the passive tissues.

The experiment began with the maximum voluntary contraction (MVC) exertions in the lumbar dynamometer. Subjects performed the maximum isometric trunk exertions at $60^{\circ}$ trunk flexion angle from upright in the sagittal plane. After completion of the MVC exertions subjects were secured to a fixture that was designed to limit pelvis rotation, and the motion sensors were secured to the skin using double sided adhesive tape and elastic bands. The fixture has two padded arms that push the pelvis backward against a fixed board so that 
the trunk flexion due to pelvis rotation could be minimized (Figure 6.4). Subjects were not allowed to bend their legs. In a training period, full flexion posture (stooped posture) was described as a slumped posture with no activity in back muscles, and upper body weight was supported passively.

Data collection consisted of two phases; a 10-minute prolonged stooping (static full flexion) phase and a 10-minute upright standing recovery phase. Before the stooping phase began, subjects performed a set of full flexion and an isokinetic lift of which observations were used as reference data points. Subjects flexed their back forward slowly and reached the full flexion posture. When the full flexion posture was achieved, subjects were asked to put their heads down, relax, and exhale (McGill and Brown, 1992), and the sagittal plane angle of this full lumbar flexion posture was measured for 5 seconds. Next, a weighted box was handed to subjects and subjects slowly lifted the box from the full flexion posture to a $30^{\circ}$ trunk flexion angle over a period of approximately 15 seconds with a consistent lifting speed. The 15-second duration for the lift was set to simulate continuous quasi-isometric weight holding postures and minimize the motion artifacts of EMG. The box dimensions were $0.3 \mathrm{~m} \times 0.3 \mathrm{~m} \times 0.25 \mathrm{~m}$ and it had two cut-out handles. The weight of the box was set at $40 \%$ of their capacity (determined from their MVC in the dynamometer). When the participant reached the $30^{\circ}$ flexion position, the experimenter took the box and the participant returned to the fully flexed posture to begin the stooping phase of the experiment.

The prolonged stooping phase started as soon as the subject finished the isokinetic lift and returned to the full flexion posture, and continued for 10 minutes. In the stooping phase, the subject maintained the relaxed full flexion posture with minimal movement of head and arms and performed the slow, isokinetic lifting task (40\% MVC) every 2.5 minutes. The full 
lumbar flexion angle was measured right before each lift and the EMG and angle were simultaneously collected during the lift. On the day with the rest break, a 30-second upright standing rest break was given at $5^{\text {th }}$ minute of stooping phase (immediately after the second isokinetic lift). On the no-rest day, this upright standing break was not provided.

At the end of $4^{\text {th }}$ lift $\left(10^{\text {th }}\right.$ minute $)$ subjects stood upright standing posture and the recovery phase began. The recovery phase continued for 10 minutes and every 2.5 minutes during this recovery period the subjects bent to the full flexion posture, held the posture for 10 seconds and then performed the slow, isokinetic lifting task (40\% MVC). Subjects returned to the upright standing posture after each lift and continued their recovery in the standing posture.

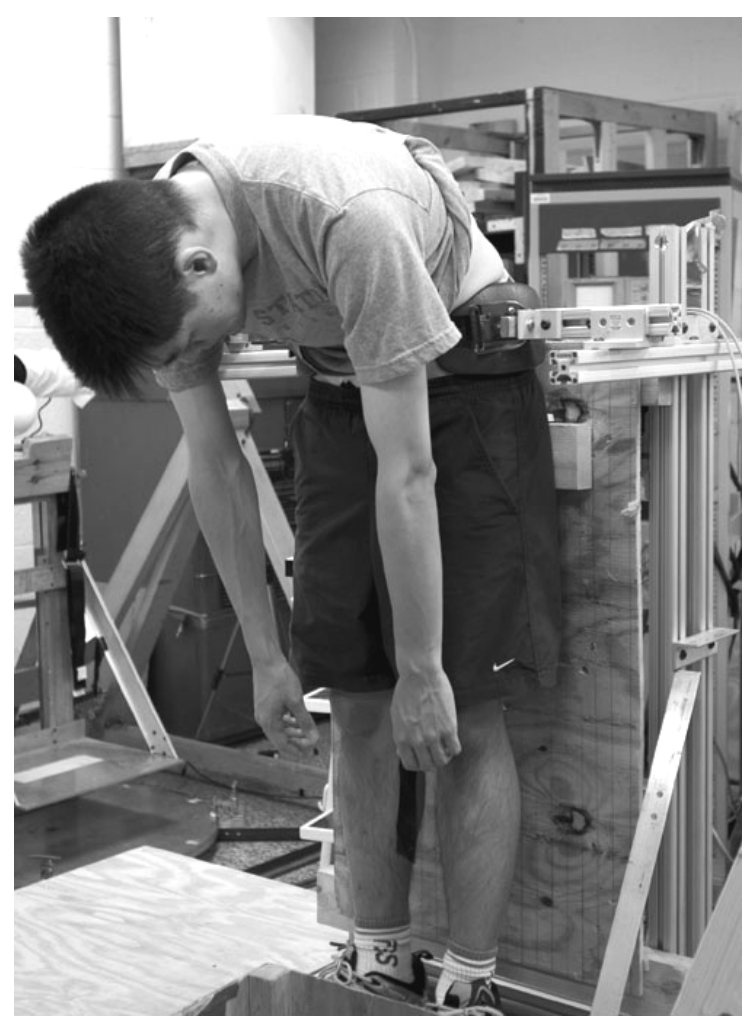

Figure 6.4. Full lumbar flexion (stooped) posture on a pelvis restraint fixture. 


\subsubsection{Experimental Variables}

\section{$\underline{\text { Independent Variables }}$}

The independent variables included the time duration of the prolonged stooping posture and recovery (TIME), and a 30 -second rest break in the middle of the stooping period (REST). Nine levels of the TIME duration ( 0 minute before stooping/ $2.5^{\text {th }}$ minute, $5^{\text {th }}$

minute, $7.5^{\text {th }}$ minute, $10^{\text {th }}$ minute in stooping period $/ 12.5^{\text {th }}$ minute, $15^{\text {th }}$ minute, $17.5^{\text {th }}$ minute, $20^{\text {th }}$ minute in recovery period), and two levels of REST break (no-rest and w/rest) were defined.

\section{Dependent Variables}

Dependent variables in this experiment included the full lumbar flexion angle in the sagittal plane and the mean EMG of the multifidus and erector spinae muscles collected during a slow isokinetic lifting task. The full lumbar flexion angle was captured for 5 seconds prior to each isokinetic lifting task and the average of the 5-second data points was used as the dependent variable. The value of the mean normalized EMG in the isokinetic lifting trial was calculated by taking an average of the linear enveloped EMG samples as the subject passed through a 2 degree trunk flexion angle window. This two degree window was related to the initial full stoop posture (this lumbar angle became the 0 degree position), and the EMG data were evaluated as the subject passed through the $1^{\circ}-3^{\circ}$ angles (relative to this $0^{\circ}$ position) during each lift. Therefore, the EMG was evaluated as the subject was assuming the same lumbar posture from trial to trial. 


\subsubsection{Data Processing}

The full lumbar flexion angle variable at each time level was simply the average of the lumbar flexion angles collected during the 5-second period just before the slow isokinetic lifting task began. The unprocessed EMG data were filtered using a 10-500 $\mathrm{Hz}$ band pass filter, a $60 \mathrm{~Hz}$ notch filter, and $42.66 \mathrm{~Hz}$ notch filters (and aliases) to eliminate noises from magnetic pulses of the motion tracker transmitter. The filtered signal was full wave rectified and filtered by $6 \mathrm{~Hz}$ Butterworth second-order low-pass filter to produce a linear envelope (Winter, 2005) and then these data were averaged over the 2-degree window of trunk flexion, as described in Section 6.2.4. The EMG data for each muscle were then normalized by dividing by the MVC EMG for that particular muscle. The normalized EMG of left and right side muscles of each muscle group were then averaged to generate one muscle normalized EMG (NEMG) for each muscle pair. Figure 6.5 shows a typical angle-EMG graph of an isokinetic lifting trial of one subject.

Finally, each data set was transposed along the Y-axis by subtracting an amount of value at Time 0 in that data set (e.g. mean full flexion angle at 0 minute in no-rest condition for the data set of the mean full flexion angle in no-rest condition) so the dependent variables at 0 minute have initial common value ' 0 ' in both rest conditions. This process was done to compare the dependent variables between rest conditions with consistent initial value (at 0 minute). 


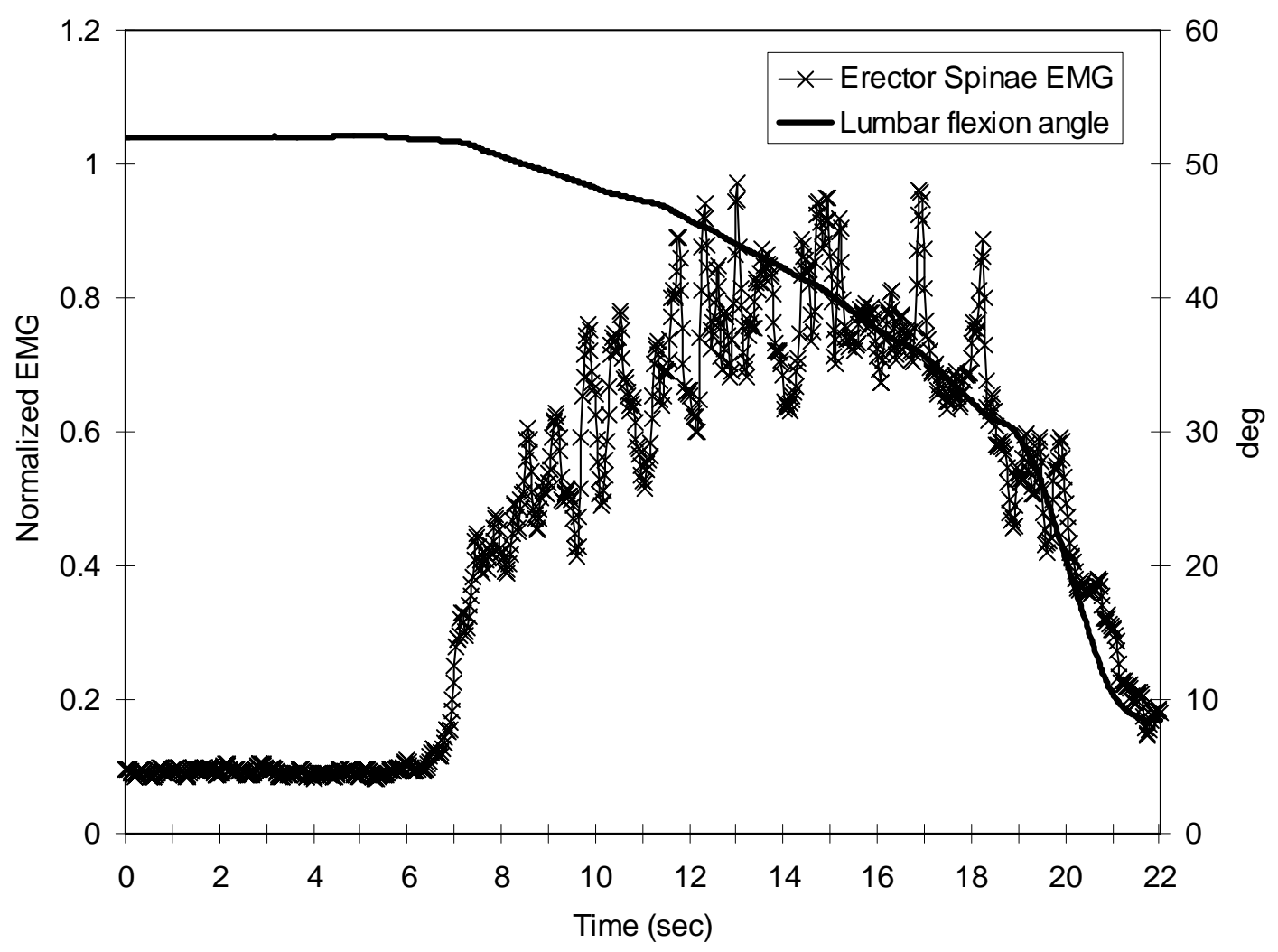

Figure 6.5. Typical angle-EMG graph of an isokinetic lifting $\left(4^{\text {th }}\right.$ lift of stooping phase).

\subsubsection{Statistical Analysis}

Multivariate Analysis of Variance (MANOVA) was conducted to assess the effects of the main (TIME, REST) and interaction (TIME*REST) terms on the set of dependent variables collectively. A two-factor randomized block design was used considering the intersubject variability as a block. The statistical model for this analysis is,

$y_{i j k}=\mu+\alpha_{i}+\beta_{j}+(\alpha \beta)_{i j}+\gamma_{k}+\varepsilon_{i j k}$ 
where $\mu$ is the overall mean effect, $\alpha_{i}$ is the effect of the $i$ th level of the TIME, $\beta_{j}$ is the effect of the $j$ th level of the REST, $(\alpha \beta)_{i j}$ is the effect of the interaction between $\alpha_{i}$ and $\beta_{j}, \gamma_{k}$ is the effect of the $k$ th block (subject), and $\varepsilon_{i j k}$ is the random error term.

The MANOVA was used prior to conducting individual ANOVAs to control for the experiment-wise error rate. Once the MANOVA identified any significant main effect or interaction effect (by the Wilks' Lambda criterion $(\mathrm{p}<0.05)$ ), univariate ANOVA tests and post-hoc Tukey tests were performed to explore these effects on individual dependent variables.

The adequacy of the models was checked by examining the normality of residuals and the equality of variance prior to any statistical analysis. The normality assumption was tested by examining normal probability plots of residuals (difference between observed value and fitted value). The equality of variance assumption was tested by plotting the residuals versus the fitted values. The normal probability plots of residuals of all dependent variables showed no severe indication of departure from normality and no possible outliers, and no apparent relationship between the residuals and the fitted values was found in the plots of the residuals versus fitted values (Appendix). These examinations suggested that the assumptions were valid and the ANOVA models were adequate (Montgomery, 2001). 


\subsection{Results}

The MANOVA tests found significant effects of both TIME, REST, and their interaction. Results of the MANOVA and following univariate ANOVA tests are listed in Table 6.2.

Table 6.2. MANOVA and ANOVA test results.

\begin{tabular}{lccc}
\hline & TIME & REST & TIME*REST \\
\hline MANOVA & $* *$ & $* *$ & $*$ \\
ANOVA & & & $* *$ \\
Full flexion angle & $* *$ & $* *$ & - \\
Multifidus EMG & $* *$ & $*$ & - \\
Erector spinae EMG & $* *$ & & \\
\hline$* \mathrm{p}<0.05, * * \mathrm{p}<0.01$ & & & \\
\hline
\end{tabular}

\subsubsection{Full Lumbar Flexion Angle}

Since the significant effect of TIME*REST interaction on the full lumbar flexion angle was found, the data set was separated by the number of levels of each independent variable and a simple effects analysis was performed. For the analysis of the TIME effect, the angle data was divided into two groups; no-rest and w/rest condition, and Tukey's pairwise comparison tests between mean values within each rest condition were performed (Table 6.3). Similarly, for the analysis of the REST effect, the data was divided into nine groups (nine TIME levels) and the mean angles between rest conditions within each time level were compared. The results of the simple effects analysis of TIME on the mean full lumbar flexion angles showed a significant effect in both rest conditions (Table 6.3 and 
Figure 6.6). The 10 -minute prolonged stooping caused a $4.2^{\circ}$ (from $44.9^{\circ}$ to $49.1^{\circ}$ ) and a $3.5^{\circ}$ (from $44.4^{\circ}$ to $47.9^{\circ}$ ) increase of the angle and the following 10-minute recovery standing resulted in $2.8^{\circ}$ (from $49.1^{\circ}$ to $46.3^{\circ}$ ) and $2.4^{\circ}$ (from $47.9^{\circ}$ to $45.5^{\circ}$ ) decrease in norest condition and $\mathrm{w} /$ rest condition, respectively. Test results exploring the simple effect of REST found that the mean full flexion angle in w/rest condition was significantly smaller $(\mathrm{p}<0.05)$ than the angle in no-rest condition at the $7.5^{\text {th }}$ minute, the $10^{\text {th }}$ minute, the $12.5^{\text {th }}$ minute, and the $15^{\text {th }}$ minute. This result indicates that the effect of the rest break was lost after ten minutes of recovery and that the simple effect of REST is not significant.

Table 6.3. Tukey's pairwise comparison between mean angle values within each rest condition (Means with different letters indicate they are significantly different).

\begin{tabular}{|c|c|c|c|c|c|c|c|c|c|}
\hline & \multicolumn{9}{|c|}{ Time level (min) } \\
\hline & 0 & 2.5 & 5 & 7.5 & 10 & 12.5 & 15 & 17.5 & 20 \\
\hline no-rest & $\mathrm{E}$ & $\begin{array}{l}\text { C } \\
\text { D }\end{array}$ & B & A & A & B & $\begin{array}{l}\mathrm{C} \\
\mathrm{B}\end{array}$ & $\begin{array}{l}\text { C } \\
\text { D }\end{array}$ & $\mathrm{D}$ \\
\hline w/rest & $\mathrm{F}$ & $\begin{array}{l}\mathrm{D} \\
\mathrm{C}\end{array}$ & B & B & A & $\mathrm{C}$ & $\begin{array}{l}\mathrm{D} \\
\mathrm{C} \\
\mathrm{E}\end{array}$ & $\begin{array}{l}\mathrm{D} \\
\mathrm{E}\end{array}$ & $\mathrm{E}$ \\
\hline
\end{tabular}




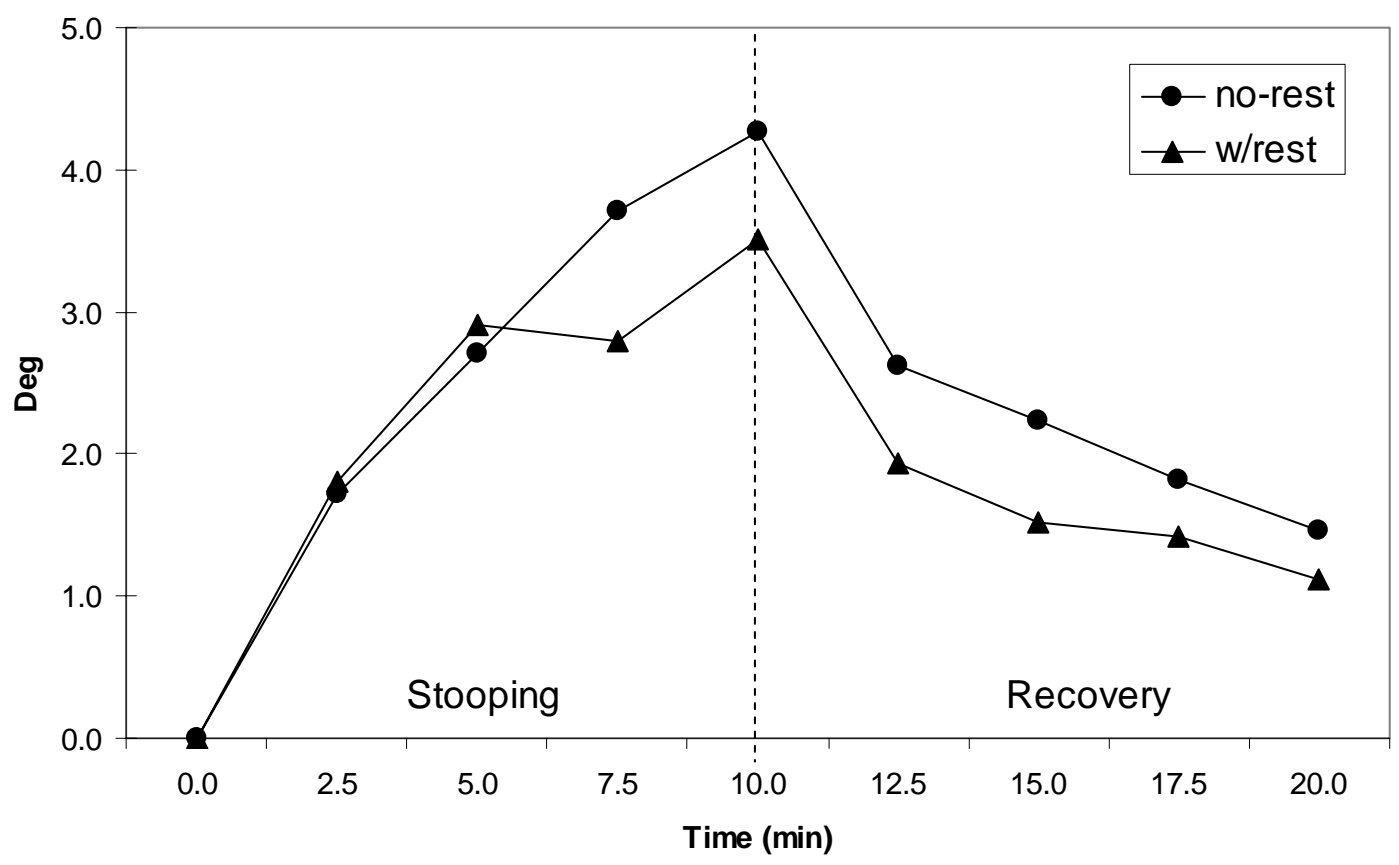

Figure 6.6. Time-dependent changes of the mean full lumbar flexion angle.

\subsubsection{EMG of Extensor Muscles}

TIME effect was significant for the mean EMG of both multifidus and erector spinae but a significant effect of the REST was observed only in the mean EMG of erector spinae. Different from the full flexion angle variable, there was no significant interaction between the REST and TIME for either EMG variable (Table 6.2).

The mean EMG of both muscles increased during stooping and decreased toward the initial level (value at Time 0) during recovery. In w/rest condition, mean EMG of erector spinae was significantly smaller than the EMG in no-rest condition. Figure $6.7 \mathrm{a}$ and $6.8 \mathrm{a}$ show time-dependent changes in the averaged mean EMG over two rest conditions and the Tukey's test results are listed in Table 6.4. Mean EMG data in each rest condition is shown 
in Figure $6.7 \mathrm{~b}$ and $6.8 \mathrm{~b}$ but no statistical analysis was performed with the separated data sets because the interaction effect (TIME*REST) was not significant by the MANOVA test.

Table 6.4. Tukey's pairwise comparison between mean EMG values within each muscle (Means with different letters indicate they are significantly different).

\begin{tabular}{ccccccccccc}
\hline & \multicolumn{8}{c}{ Time level (min) } \\
\cline { 2 - 10 } & 0 & 2.5 & 5 & 7.5 & 10 & 12.5 & 15 & 17.5 & 20 \\
\hline $\begin{array}{c}\text { Multifidus } \\
\text { EMG }\end{array}$ & E & B & B & A & A & A & C & C & E & E \\
\hline $\begin{array}{c}\text { Erector Spinae } \\
\text { EMG }\end{array}$ & F & B & B & A & B & A & B & E & E & E \\
& & & & & & D & D & D & F \\
\hline
\end{tabular}




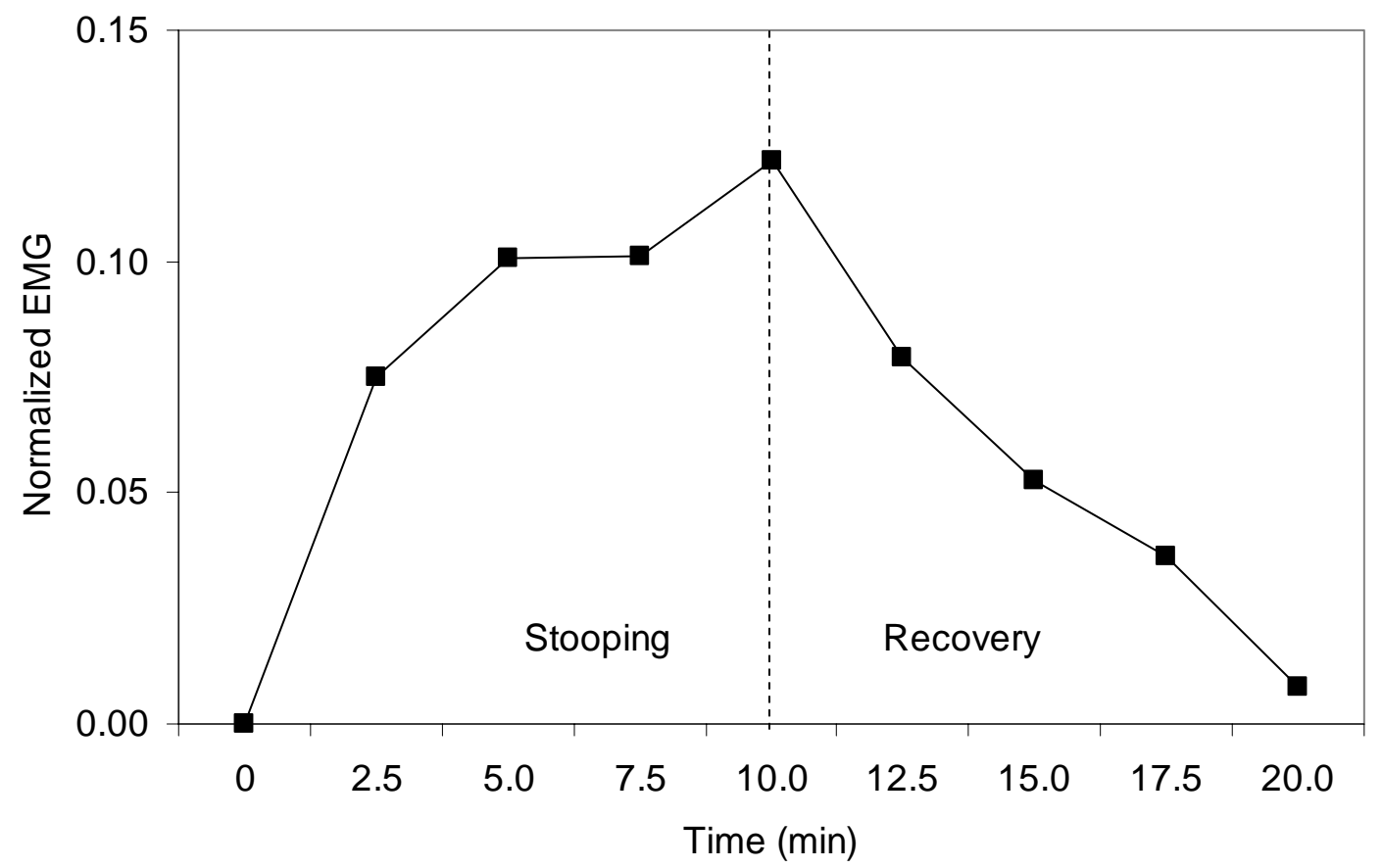

(a)

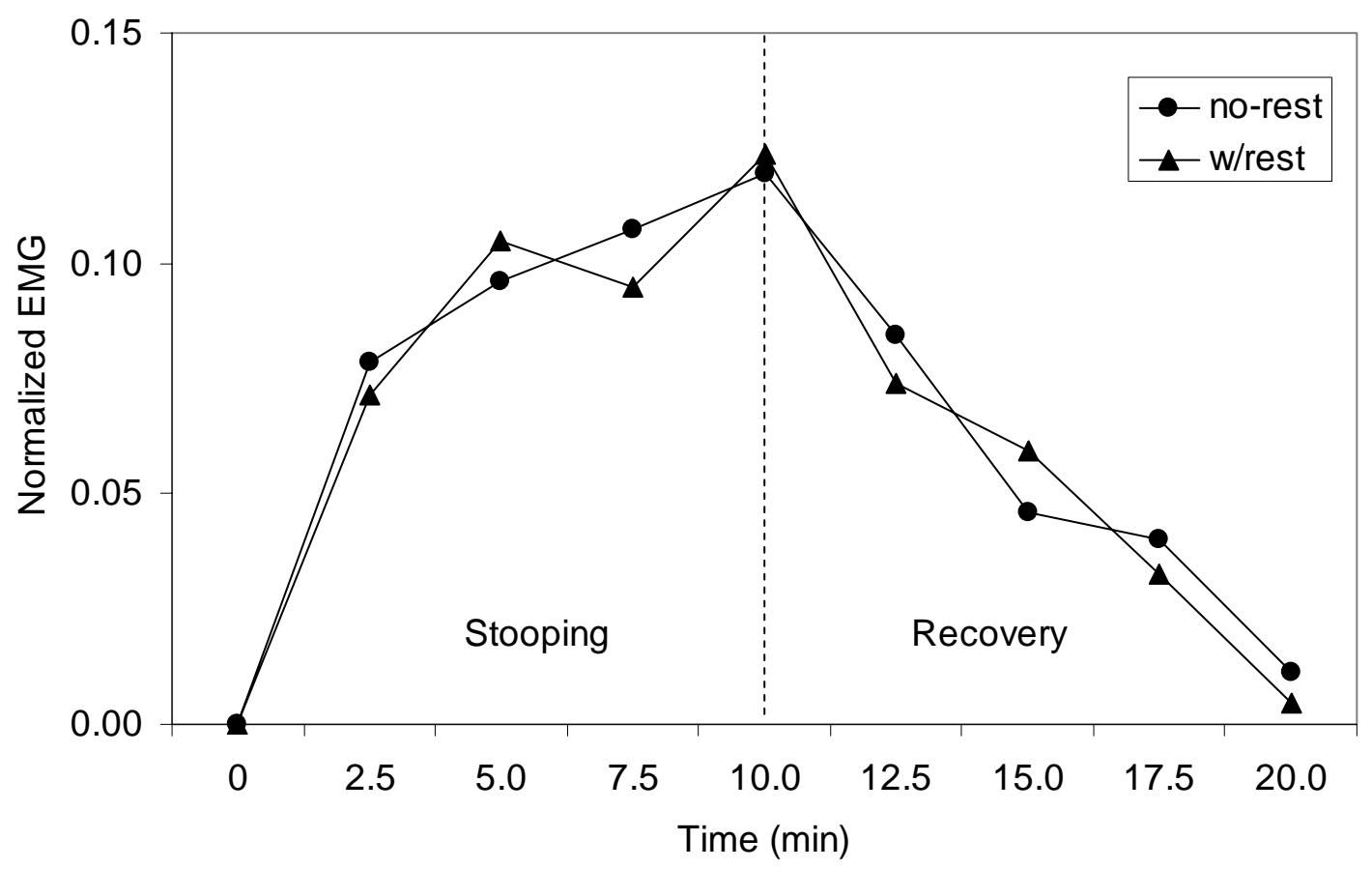

(b)

Figure 6.7. Time-dependent changes of the mean EMG of multifidus. a) average of both rest conditions, b) in each rest condition. 


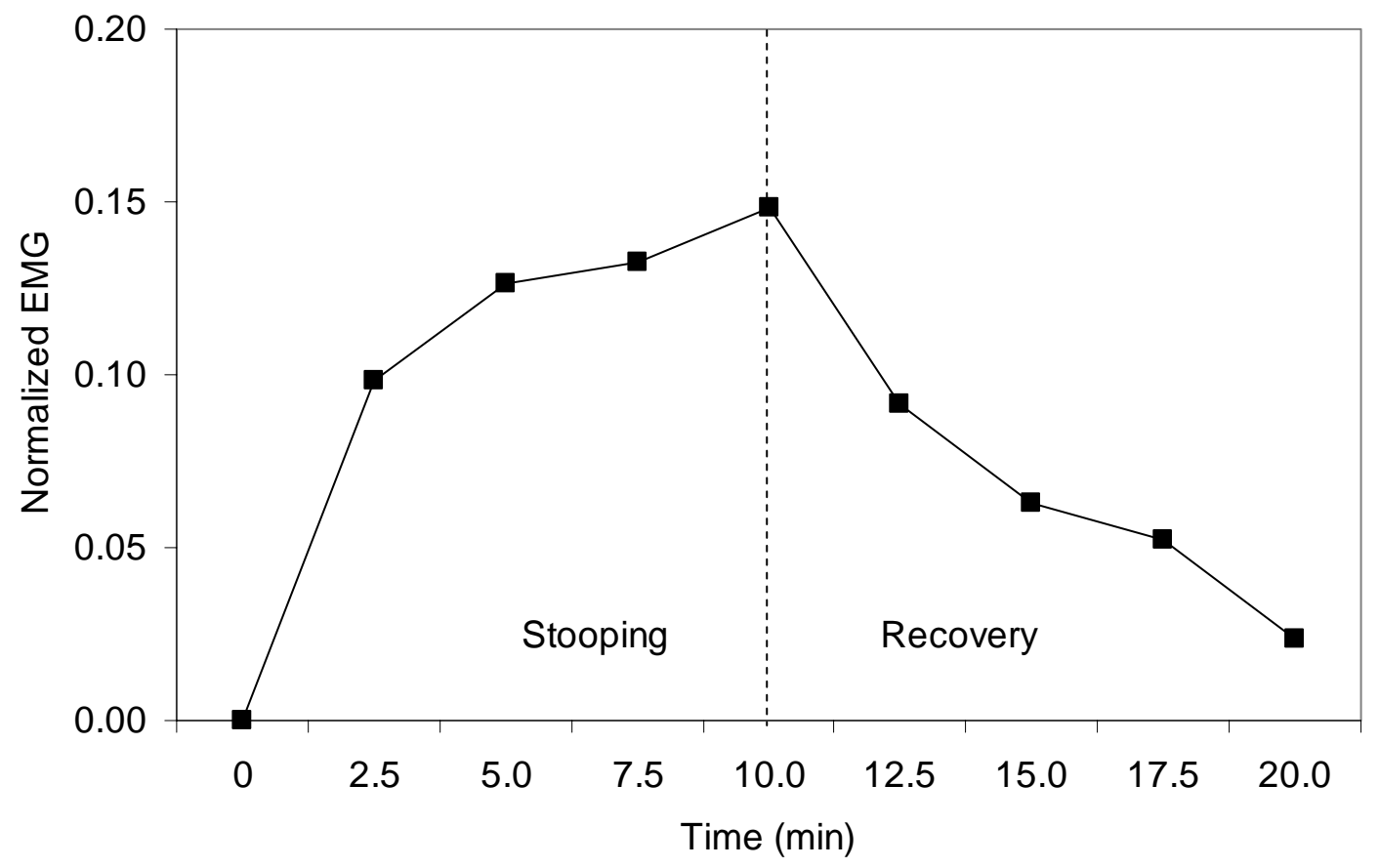

(a)

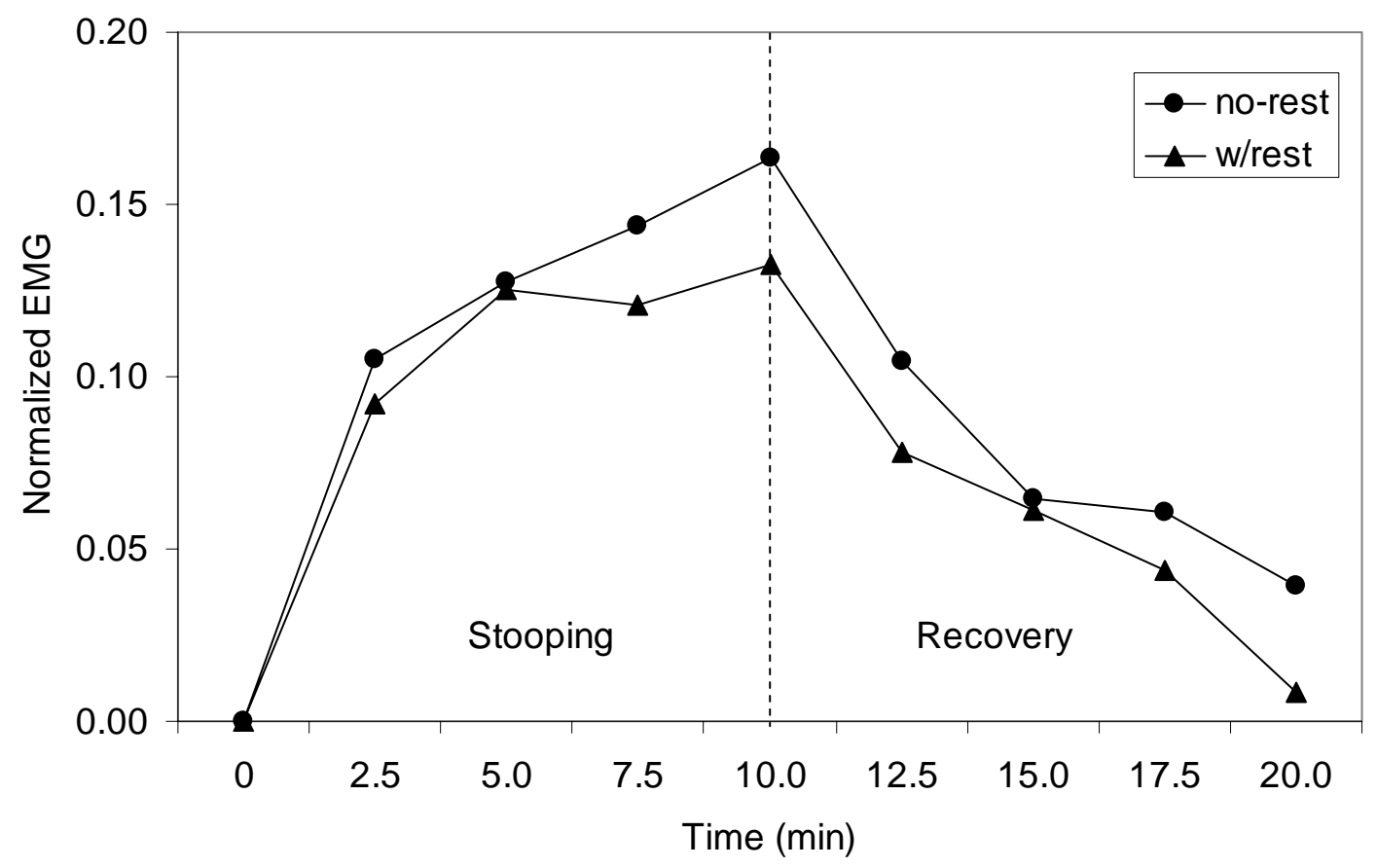

(b)

Figure 6.8. Time-dependent changes of the mean EMG of erector spinae. a) average of both rest conditions, b) in each rest condition.. 


\subsection{Discussion}

The two main issues addressed in this experiment were how the creep deformation in the lumbar spine over a time period of prolonged stooping and recovery affects the extensor muscle activity level in slow isokinetic lifting tasks, and how does a short (30 seconds) micro-break in this prolonged stooped posture affect the creep deformation and the muscle activity responses. As has been shown in previous work, the full flexion angle was expected to increase in stooping period and decreased in recovery period due to viscoelastic material properties of passive tissues. The changes in the passive tissue stiffness was expected to affect the activation level of back extensor muscles because the flexion moment by upper body weight and/or external load on hands is supported by co-contribution of both active and passive components. It was also expected that the micro-break in the middle of stooping was expected to moderate those effects, and these expectations were realized in this experiment.

Results for the full lumbar flexion angle showed significant increases during the stooping phase of the study. The 10-minute stooping generated an average increase of $4.2^{\circ}$ and $3.5^{\circ}$ of the full lumbar flexion angle in no-rest condition and w/rest condition, respectively. The magnitude of the increase of the angle in the 10-minute prolonged stooping was comparable to other earlier in vivo studies. A model by McGill and Brown (1992) predicted $4.06^{\circ}$ increase of the angle during a 10 -minute static full flexion exertion. In their study, the full flexion angle before stooping was observed as $67.3^{\circ}$ and this was relatively greater than the angle of the current study (mean $44.6^{\circ}, \mathrm{SD} 3.6^{\circ}$ ). This might be due to differences in the initial postures for reference angle (neutral upright posture) between the two studies. The reference angle in the current study was measured when subjects were 
standing upright on a pelvis restraint system with secured pelvis, while McGill and Brown (1992) measured it when subjects sat relaxed leaning against a backrest.

Greater full lumbar flexion angle after prolonged stooping indicated creep deformation of passive tissues and this implies less contribution of passive tissues to generating extension moment in weight holding or lifting tasks. Transferring extension moment from passive tissues to active muscles was observed by the analysis of EMG of back extensors. Changes in the normalized EMG in stooping and recovery resembled the responses of the full lumbar flexion angle. In the 10-minute stooping phase of the experiment, the normalized EMG of multifidus and erector spinae muscles increased an average of $28.6 \%$ and $39.3 \%$, respectively, suggesting that muscles were required to generate more active forces to compensate for the loss of contribution of passive tissues. Adams and Dolan (1996) examined the time-dependent changes of the resistance to bending in the lumbar spine passive tissues by cadaver lumbar segments. Bending moment on the centre of the disc was computed when the lumbar spine motion specimen was flexed to $70 \%$ of flexion limit and held for 5 and 60 minutes. Average decrease in the bending moment during the sustained flexion was $42.3 \%$ and $66.8 \%$ after 5 and 60 minutes, respectively. Since their specimens did not include the passive component of muscle-tendon units, the decrease in the bending moment was mostly attributable to the viscoelastic responses of the posterior ligaments and the intervertebral disc. In the in vivo situation, the active contribution of the back muscles to generating extension moment should increase during sustained or prolonged flexion, as shown in the current study. The shift of extension moment from posterior ligaments to active muscles has also been observed in Shin et al. (2004). EMG activities of the back extensor muscles were monitored during static weight holding tasks with different 
levels of knee and trunk flexion angle. As knee angle increased from $0^{\circ}$ to $40^{\circ}$ at $90^{\circ}$ trunk flexion angle, the normalized EMG increased significantly from relaxation level and the effect of knee angle was more pronounced for high-flexible subject group. In static trunk flexion posture, the tension in the bi-articular biceps femoris, which was influenced by knee flexion and flexibility, affected the tension of posterior ligaments via pelvic rotation and finally resulted in the change of ratio of contribution of active muscles to passive tissues in generating extension moment.

The greater full lumbar flexion angle after stooping indicates more slack in the lumbar spine, and the greater muscle activity level was a sign of reduced contribution of passive tissues in generating extension moment. Lumbar spine stability could be compromised by these mechanical responses and the elevated instability in the lumbar spine has been known to affect the intervertebral disc pressure and the muscular activity level and finally result in pain or disorders in low back (Solomonow, 2004).

The 10-minute recovery after the prolonged stooping was not enough for full recovery of full lumbar flexion angle and this was consistent with earlier studies. This slow recovery might be due to the four repetitions of full flexion and lifting tasks that occurred every 2.5 minutes during the recovery phase (McGill and Brown, 1992), but a study with a feline spine model also showed that creep by 10 -minute flexion was not fully recovered by 10-minute rest period and large cumulative creep was observed at the end of cycles of flexion-rest periods (Solomonow et al., 2003b). This result indicated the importance of sufficient rest break or recovery period between consecutive stooping tasks in minimizing the cumulative creep and tissue damage in the lumbar spine. The duration and frequency of rest break within a work day, and the amount of recovery period between consecutive work days 
are key components that have influence on the development of cumulative stress and/or fatigue on low back. It is important to examine how the rest break parameters are related to the low back mechanics and physiology in designing work-rest cycles that can minimize the cumulative risks for LBDs from repetitive prolonged stooped postures.

The normalized EMG of extensor muscles showed faster decrease than the full flexion angle during the recovery and this might be related to the reduced EMG and forcegenerating capacity of back extensors that have been observed after passive stretch of human leg muscles (McHugh et al., 1992; Fowles et al., 2000; Avela et al., 2004). Together with strained passive tissues, the reduced mechanical capacity of the back extensors could reduce the safety margin in the lumbar spine and finally result in serious damage in the low back, especially when high exertion is required immediately after prolonged stooped posture such as standing up or lifting a weight from fully flexed posture or seated posture.

The 30-second rest break moderated the creep of the full lumbar flexion angle and resulted in a significantly less full flexion for the following 10 minutes than the condition without the rest break. This indicated the benefit of the micro-break during prolonged stooping tasks as it could reduce the cumulative strain of passive tissues in prolonged stooping. However, the benefit of the break to the muscle activity level was not as strong as to the cumulative creep because the significant difference in EMG between conditions was not observed consistently. This inconsistent result might be due to the nonlinear stress-strain relationship of the passive tissues. Figure 6.9 shows typical stress-strain curves of a ligament before (0 minute) and after creep loading (A minute, B minute, $\mathrm{B}>\mathrm{A}$ ) (Nachemson and Evans, 1968; Dumas et al., 1987; Yahia et al., 1991). The change of the full flexion angle during stooping is related to the creep deformation of the ligaments under constant stress (from 'R0' 
to 'RA' and 'RB'), and the change of the mean EMG is related to the stress level about $2^{\circ}$ above the initial full flexion angle (from ' $\mathrm{S} 2$ ' to 'SA' and 'SB'). Because of the nonlinear relationship, the ratio of the strain increase $(|\mathrm{RA}-\mathrm{RB}| / \mathrm{R} 0-\mathrm{RA} \mid)$ between the curves is greater than the ratio of the stress increase $(|\mathrm{SA}-\mathrm{SB}| /|\mathrm{S} 2-\mathrm{SA}|)$. This indicates the less sensitive response of the EMG than the full flexion angle after a certain amount of creep has occurred, and it might result in the inconsistent effects of the rest break on the dependent variables in this study. This also could explain the relatively greater increase of the EMG than the full flexion angle during the first 2.5 minutes stooping period (Figure 6.7 and 6.8). 


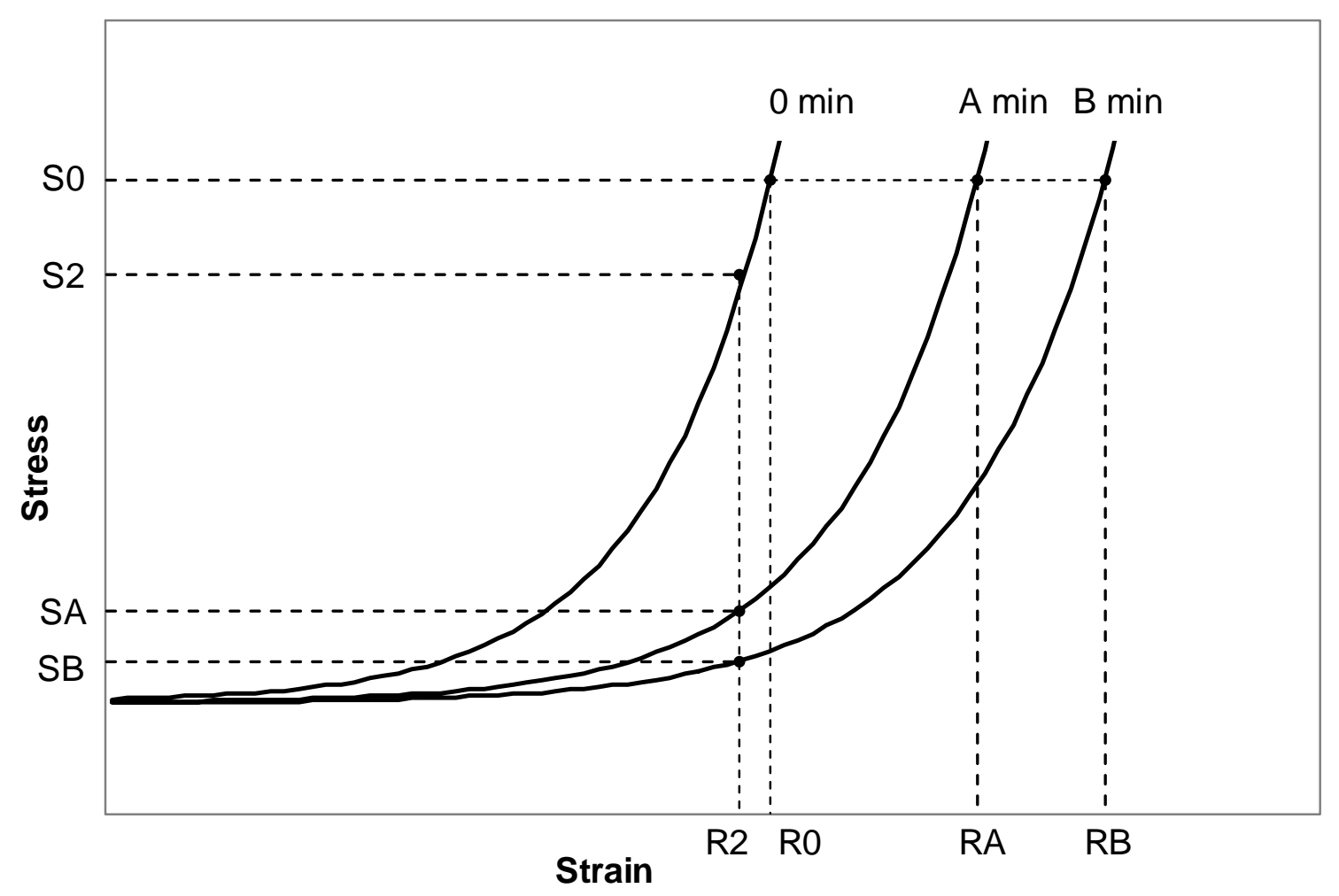

Figure 6.9. Time-dependent changes of the stress-strain curve of a ligament during stooping. SO : stress at the full flexion angle.

S2 : stress at $2^{\circ}$ above the initial full flexion angle at time ' 0 '.

$\mathrm{SA}$ : stress at $2^{\circ}$ above the initial full flexion angle at time ' $A$ '.

SB : stress at $2^{\circ}$ above the initial full flexion angle at time ' $B$ '.

$\mathrm{RO}$ : strain at full flexion angle at time ' 0 '.

$R 2$ : strain at $2^{\circ}$ above the initial full flexion angle.

RA : strain at full flexion angle at time ' $A$ ' $(A>0)$.

$R B$ : strain at full flexion angle at time ' $B$ ' $(B>A)$.

There were some experimental limitations that need to be recognized. First, subjects in this study extended their back four times during stooping phase to perform isokinetic lifts and the extension might provide a chance for recovery of the passive tissues. However, since the full extension was not achieved at the end of each lift (stopped at $30^{\circ}$ flexion), the recovery of passive tissues in these extensions might not be as efficient as in full upright standing posture. Had these short breaks not been provided, the effects shown might have 
even been more pronounced. Second, the duration of stooped posture in this experiment was determined to minimize subject discomfort and the possibility of injury from the experiment. Although the 10-minute period was sufficient to generate significant viscoelastic responses of the lumbar spine, it might not be enough to represent the common work duration in industries. Workers who have their major tasks under knee would spend most of their work time in stooped posture with intermittent short extension and rest breaks. It is reasonable to expand this basic research to applicable study with extended duration of the stooped posture to examine cumulative viscoelastic responses in longer period of the stooping. Third, the stooped posture in industry might be more dynamic and involve frequent over flexion or mild extension as the height of the work point changes. Since this study employed a quiet static posture during stooping, the generalizability to real occupational tasks is somewhat limited. But, since the dynamic or quasi-dynamic lumbar motion in stooping was believed to exert greater load on the passive tissues due to additional flexion moment from its moment of inertia and acceleration, the mechanisms between the prolonged stooping and elevated risks for LBDs are still valid.

There are a number of risk assessment tools for low back including the NIOSH Revised Lifting Equation (Waters et al., 1999), the University of Michigan 3-D Static Strength Prediction Program (Chaffin et al., 1999), the Ohio State University Lumbar Motion Monitor Model (Marras et al., 1995), and the Texas Tech University Job Severity Index (Ayoub et al., 1983). Most of these tools are focused on quantifying risks in traditional manual material handling tasks such as lifting and lowering, but they are not capable of predicting time-dependent and cumulative risks from the prolonged stooped posture. Based on the results of this basic study, the next step in this research will be to develop a 
biomechanical model to quantitatively predict the time-dependent changes in the lumbar spine. This model can be used as a tool to assess risks of work-related prolonged stooped postures and help reduce work-related LBDs.

Risk assessment of prolonged stooping requires an understanding of mechanical changes in each passive tissue as well as the gross response of the lumbar spine. Prolonged stooping produces multiple risk factors for LBDs, such as tissue damage and/or failure due to excess strain or stress, reduced mechanical capacity of back extensors, and possibly instability in the spine. The severity of those risks can be affected by some internal and external factors including age, gender, anthropometry, time duration of stooping and recovery, micro-break, and weight of external load. Understanding how individual tissues respond to combinations of various factors is critical to assess the risk of prolonged stooping in industries. The development and validation of this biomechanical model is the focus of the next paper (Chapter 7) presented in this dissertation.

\subsection{Conclusions}

The viscoelastic responses of the lumbar spine in terms of full lumbar flexion angle and EMG of back extensor muscles were examined to improve our understanding of the interplay between the passive and active extensor mechanisms, with a long-range goal of better understanding the risks for low back disorders due to prolonged stooping.

Both the full lumbar flexion angle and the EMG of back extensors in lifting were significantly affected by the time duration of stooping and recovery. The 10-minute prolonged stooping caused a $4.2^{\circ}$ and a $3.5^{\circ}$ increase of the angle and the following 10 minute recovery standing resulted in $2.8^{\circ}$ and $2.4^{\circ}$ decrease in no-rest condition and w/rest 
condition, respectively. The EMG of back extensor muscles also increased 28.6\%-39.3\% during the 10-minute stooping period. These results indicated creep and stress-relaxation of passive tissues in the lumbar spine, and the transfer of the extension moment between the passive tissues and active muscles as a result of the stooping and recovery duration.

These mechanical changes of the passive tissues are known to degrade lumbar spine stability and it requires greater contribution of muscles to sustain the stability and support flexion moment. Since the passive stretching of muscle-tendon units and neighboring ligaments in fully flexed posture could lead to the reduction of functional capacity of muscles, tasks that require substantial amount of muscle exertion immediately after prolonged stooping, such as lifting a filled bucket after picking up crops in agriculture field, could result in acute injury or disorder in the low back. Results of this study also suggested that the risk of prolonged stooping could be moderated by taking a short period of rest break in the middle of stooping period. 


\section{FINITE ELEMENT MODEL SIMULATIONS}

\subsection{Introduction}

The stooped or fully flexed posture maintained over a prolonged period of time has been shown to influence the biomechanics and physiology of the lumbar spine tissues and it has been suggested that the changes in the spinal tissues can contribute to the development of low back disorders (LBDs) or pain (LBP) in work-related tasks (Solomonow et al., 2003b; Solomonow, 2004; McGill, 1997).

The changes in the lumbar spine during stooping are mainly attributed to the viscoelastic behaviors of the spinal tissues and interaction between passive tissues and active muscles in extension moment generation. In the stooped posture, the upper body weight is supported mainly by passively generated extension moment from the spinal ligaments and muscle-tendon units in the posterior side of the spine (Kippers and Parker, 1984; Schultz et al., 1985; McGill and Kippers, 1994). Since most spinal tissues are viscoelatic, continuous loading during prolonged stooping can initiate creep deformation (in constant stress) and stress-relaxation (in constant strain) of the passive tissues (Solomonow et al., 2003b; Adams and Dolan, 1996). In addition, the passive stretch over a prolonged period can cause microdamage and acute inflammation of the ligaments. These mechanical and physiological changes in the passive tissues can impair lumbar spine stability and cause the surrounding muscles to generate more active forces to maintain spinal stability and protect the damaged tissues (Solomonow et al., 2003b). However, the passively stretched back extensor muscles in the stooped posture may lose their force-generating capacity (Fowles et al., 2000; Avela et al., 2004), leading to reduced strength performance of back extensor muscles. Greater demand but lower performance of active muscles in a situation of impaired spinal stability 
may result in faster muscle fatigue generation or failure to sustain lumbar stability, specifically when the lumbar spine is subjected to high external load immediately after the prolonged stooping.

Identifying variables impacting the relationship between the prolonged stooped posture (e.g. duration, rest-breaks, etc.) and the viscoelastic mechanical response (e.g. creep response, muscle activation profiles, etc.) is an important step to understand the injury mechanism and assess risks of the prolonged stooping for LBDs. A biomechanical model, such as a finite element (FE) model of the lumbar spine, can be employed for this purpose.

The lumbar spine is a complicated structure consisting of many soft tissues of which material properties are nonlinear and viscoelastic. Due to the complexity of the structure and difficulties with incorporating many internal/external variables, computational modeling and simulation using a FE model can be a useful method to quantify responses of the lumbar spine during prolonged stooping.

For decades, finite element analysis (FEA) has been used for modeling human vertebral motion segments. A two-dimensional (2D) axisymmetric model by Belytschko et al. (1974) was one of the earliest FE models of the human spine motion segment. The model considered incompressibility of the nucleus pulposus and orthotropic elastic modulus of the annulus fibrosus. The nonlinearity of the orthotropic material constants of the annulus fibrosus was introduced by Kulak et al. (1976). They compared the linear and nonlinear material properties of the annulus fibrosus and suggested that nonlinear material properties need to be included in the model of the intervertebral disc. A more realistic threedimensional (3D) FE model was created by Shirazi-Adl et al. (1984). They modeled the annulus fibrosus as a composite of two different elements: annulus ground substance in eight 
layers and collagenous fibers that embedded in each layer of the annulus with an average angle of $29^{\circ}$ to the horizontal plane. Their revised model (Shirazi-Adl et al., 1986) included nonlinear spinal ligaments to examine the function of ligaments in flexion. All ligaments had an identical elastic modulus for the linear region due to lack of material property information but each had a different length for the toe region.

Recent FE models have incorporated viscoelastic material properties for the disc components and spinal ligaments to simulate time-dependent behaviors of the lumbar motion segment (Lu et al., 1998; Wang et al., 2000; Wang et al., 1997; Cheung et al., 2003). Viscoelastic material properties of disc components and spinal ligaments from in vitro tissue experiments were incorporated into the FE models to study the viscoelastic responses of a single motion segment under constant creep compression load (Wang et al., 1997; Lu et al., 1998; Cheung et al., 2003), vibrational compression load (Cheung et al., 2003), and static sagittal flexion load with varying flexion speed (Wang et al., 2000). Results of these models suggested that consideration of the viscoelastic material properties of passive tissues is essential to studying mechanical behaviors of a motion segment (Wang et al., 2000).

Spinal ligaments and extensor muscles are two major components that maintain lumbar stability, support upper body weight, and drive the changes in the lumbar spine during prolonged stooping. A study of prolonged stooping requires the creation of a $3 \mathrm{D} \mathrm{FE}$ model of the lumbar spine with viscoelastic ligaments and extensor muscles in order to examine time and posture-dependent changes in the relationship between ligaments and muscles in maintaining lumbar stability and supporting upper body weight as well as global geometry of the lumbar spine. Previous FEA models have looked at viscoelastic responses of the lumbar spine without muscles (Wang et al., 1997; Wang et al., 2000; Cheung et al., 
2003; Lu et al., 1998) or elastic behaviors with muscles (Zander et al., 2001; Goel et al., 1993; Shirazi-Adl et al., 2002). No FE model of a whole lumbar spine with full nonlinear and viscoelastic material properties has been created for the analysis of mechanical responses during prolonged stooping.

The objective of this study was to 1) develop a 3D nonlinear viscoelastic FE model of a whole lumbar spine that could simulate prolonged stooped and upright standing recovery posture and evaluate time-dependent mechanical responses of the lumbar spine, and 2) validate the model using empirical data from human subjects performing a prolonged stooped exertion. It was hypothesized that the model would predict that full lumbar flexion angle would increase during prolonged stooping and decrease during recovery due to creep deformation of passive tissues. Similarly, muscle force demand for a weight holding was expected to increase during stooping and decrease during recovery because of stressrelaxation of the passive tissues. These predictions were then compared with the empirical data to validate the modeling approach. The validated model may be used further to estimate the risks of prolonged stooping and suggest a proper duty cycle to minimize cumulative creep deformation of the passive tissues and stress/load on the lumbar spine.

\subsection{Methods}

7.2.1. Geometry and Materials

A 3D nonlinear viscoelastic FE model of the lumbar spine consisting of five vertebrae (L1 to L5), five intervertebral discs (L1/L2 to L5/S1), posterior ligaments (posterior longitudinal ligament, PLL; capsular ligament, CL; ligament flavum, LF; intertransverse ligament, ITL; interspinous ligament, ISL; supraspinous ligament, SSL), and lumbar extensor 
muscles (multifidus, erector spinae) was created (Figure 7.1). Since geometry and boundary conditions are symmetric to the mid-sagittal plane, only the right half of the lumbar spine was created and symmetry boundary conditions were applied to the mid-sagittal plane. Finite element software ABAQUS v6.4 (ABAQUS, Inc. Providence, RI) was used for pre- and postprocessing.

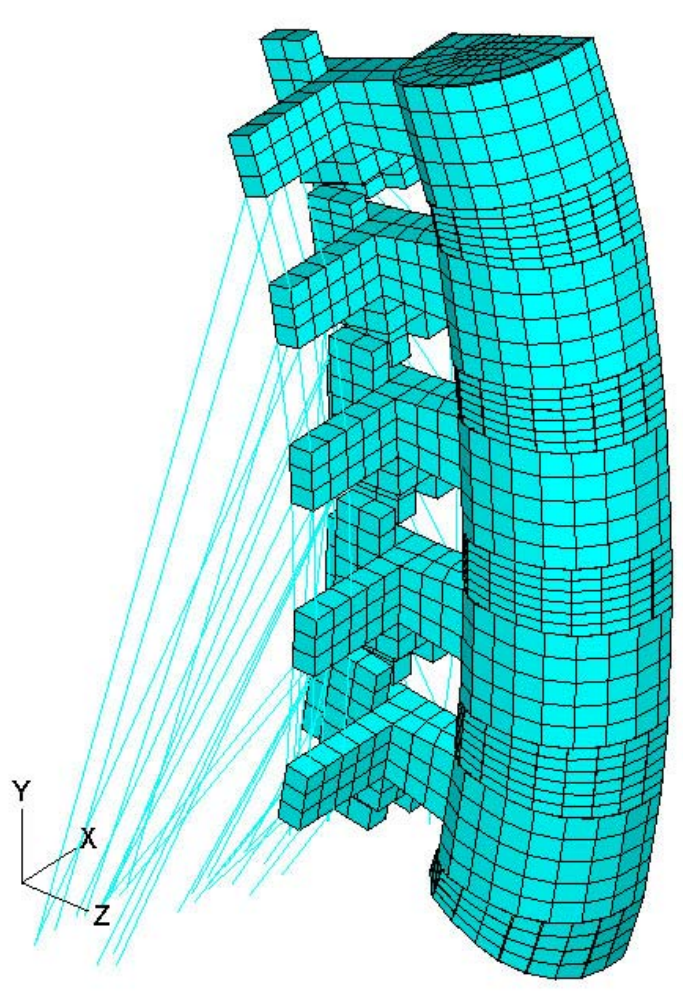

Figure 7.1. 3D FE model of lumbar spine.

\section{Vertebra}

The 3D geometry of the vertebrae was defined based on the quantitative measurements of L3 vertebrae of cadaver spines (Panjabi et al., 1992) (Table 7.1). 
Dimensional variation along a vertical location was not considered. The vertebrae were modeled as a linear elastic material because the magnitude of viscoelastic strain of bone is negligible compared to the responses of other soft tissues ( $\mathrm{Lu}$ et al., 1998). Load transmission through facet joint contact was not considered in this model because sagittal flexion caused negligible contact forces between adjacent facet surfaces (Shirazi-Adl et al., 1986; Shirazi-Adl, 1994). Material properties of the bones and other spinal tissues are listed in Table 7.2.

Table 7.1. Dimensions of the vertebral body and the disc (CSA : Cross-sectional area, PCSA : Physiological cross-sectional area).

Vertebral body height (mm)

Vertebra top/bottom surface area $\left(\mathrm{mm}^{2}\right)$

Anterior-posterior disc diameter (mm)

Lateral disc diameter $(\mathrm{mm})$

Cortical shell thickness (mm)

Annulus fiber CSA $\left(\mathrm{mm}^{2}\right)$

Ligaments CSA $\left(\mathrm{mm}^{2}\right)$

PLL / LF / CL / ITL / ISL / SSL

Muscles PCSA $\left(\mathrm{mm}^{2}\right)$

multifidus from L1/L2/L3/L4/L5

iliocostalis from $\mathrm{L} 1 / \mathrm{L} 2 / \mathrm{L} 3 / \mathrm{L} 4$

longisimus from L1/L2/L3/L4/L5
24

1428

35

46

1

0.2

14.4 / 75 / $36 / 10 / 40 / 30$

44.5 / 44.2 / $52.7 / 46.5$ / 22.5

$108 / 154 / 182 / 189$

79 / $91 / 103 / 110 / 116$ 
Table 7.2. Element types and material properties.

\begin{tabular}{|c|c|c|c|c|c|}
\hline & Element type & $\begin{array}{l}\text { Aspect } \\
\text { ratio }\end{array}$ & $\begin{array}{c}\text { Elastic modulus } \\
(\mathrm{MPa})\end{array}$ & $\begin{array}{l}\text { Poisson } \\
\text { ratio }\end{array}$ & References \\
\hline Cortical Shell & 8-node brick & $<6.51$ & 12,000 & 0.3 & \\
\hline Cancellous core & 8-node brick & $<3.56$ & 100 & 0.2 & Wang et al. (1997), \\
\hline Bony elements & 8-node brick & $<2.17$ & 7,000 & 0.25 & Goel et al. (1995), \\
\hline Annulus matrix & 8-node brick & $<4.75$ & 4.2 & 0.45 & (1986) \\
\hline Nucleus & 8-node brick & $<3.28$ & 1 & 0.495 & Lu et al. (1998) \\
\hline Annulus fibers & $\begin{array}{l}\text { 4-node surface } \\
\text { with rebar layers }\end{array}$ & - & 450 & 0.3 & \\
\hline Ligaments & 2-node truss & - & $0.5-70$ & - & - \\
\hline Muscles & hyperelastic & - & $0.1-19$ & - & - \\
\hline
\end{tabular}

\section{Intervertebral discs}

Five intervertebral discs were modeled with varying thickness and wedge angle to form a lordotic profile of the lumbar spine at neutral upright standing posture. The exterior height and the wedge angle of each disc in sagittal plane were obtained from studies of cadaver CT scan (Breau et al., 1991; Shirazi-Adl, 1994) (Table 7.3). The intervertebral disc consists of three components: nucleus pulposus, annulus matrix, and annulus fibers. The nucleus pulposus was modeled as an isotropic linear elastic material with a Poisson's ratio close to $0.5(v=0.495)$ to represent its incompressible fluid properties under static loading (Iatridis et al., 1996). The annulus matrix was modeled as an isotropic linear viscoelastic material. Best et al. (1994) measured uniaxial creep displacement of the annulus fibrosus under a compression load with constraining radial directional deformation. The creep displacement was transformed to the reduced relaxation function of bulk modulus $K(t)$, defined by 
$K(t)=K_{0}\left(1-\sum_{i=1}^{n} K_{i}\left(1-e^{-t / \tau_{i}}\right)\right)$

where $t=$ time, $K_{0}$ is the instantaneous elastic modulus at $t=0, K_{i}$ is the Prony series weighing factor, and $\tau_{i}$ is the relaxation time constant. The Prony constants were input to ABAQUS (Table 7.4).

Table 7.3. Dimensions of the cross-section of the intervertebral discs in sagittal plane (CSA : Cross-sectional area).

\begin{tabular}{|c|c|c|c|c|c|}
\hline & \multicolumn{2}{|c|}{$\mathrm{CSA}\left(\mathrm{mm}^{2}\right)$} & \multicolumn{2}{|c|}{ Exterior Height (mm) } & \multirow{2}{*}{$\begin{array}{l}\text { Wedge Angle } \\
\text { (deg) }\end{array}$} \\
\hline & Annulus & Nucleus & Anterior & Posterior & \\
\hline $\mathrm{L} 1 / \mathrm{L} 2$ & 200.6 & 186.5 & 14.3 & 7.9 & 10.8 \\
\hline $\mathrm{L} 2 / \mathrm{L} 3$ & 218.4 & 204.4 & 14.2 & 10.2 & 6.2 \\
\hline $\mathrm{L} 3 / \mathrm{L} 4$ & 456.5 & 221.0 & 14.5 & 11.6 & 5.1 \\
\hline L4/L5 & 247.1 & 231.1 & 16.1 & 11.3 & 8.0 \\
\hline $\mathrm{L} 5 / \mathrm{S} 1$ & 181.3 & 166.6 & 15.0 & 5.1 & 15.6 \\
\hline
\end{tabular}

The annulus matrix has six layers in the radial direction (Goel et al., 1995) and each layer contained tension-only annulus fibers completely embedded in a matrix $\pm 30^{\circ}$ from the horizontal mid-plane of the disc. The fibers occupied approximately $20 \%$ of the volume of the annulus fibrosus (Marchand and Ahmed, 1990; Goel et al., 1995). The viscoelastic property of the annulus fibers was derived from experimental results of stress-relaxation tests of tendon tissue (Sarver et al., 2003; Wang et al., 1997). The same Prony constants were used for muscle elements as well as annulus fibers. 
Table 7.4. Prony series constants for viscoelastic elements (acquired by curve fitting to experimental data).

\begin{tabular}{lllll}
\hline & Annulus matrix & Annulus fibers & Ligaments & Muscle-tendon \\
\hline & $g_{1}=0$ & $g_{1}=0.062$ & $g_{1}=0.7045$ & $g_{1}=0.062$ \\
Shear $(\mathrm{G}(\mathrm{t}))$ & $g_{2}=0$ & $g_{2}=0.042$ & $g_{2}=0.107$ & $g_{2}=0.042$ \\
& $g_{3}=0$ & $g_{3}=0.065$ & $g_{3}=0.076$ & $g_{3}=0.065$ \\
& $g_{4}=0$ & $g_{4}=0.15$ & $g_{4}=0.1102$ & $g_{4}=0.15$ \\
& & & & \\
& $k_{1}=0.1317$ & $k_{1}=0$ & $k_{1}=0$ & $k_{1}=0$ \\
Bulk (K(t)) & $k_{2}=0.4034$ & $k_{2}=0$ & $k_{2}=0$ & $k_{2}=0$ \\
& $k_{3}=0.1893$ & $k_{3}=0$ & $k_{3}=0$ & $k_{3}=0$ \\
& $k_{4}=0.1581$ & $k_{4}=0$ & $k_{4}=0$ & \\
& & & & $\tau_{1}=1$ \\
& $\tau_{1}=8$ & $\tau_{1}=1$ & $\tau_{1}=1$ & $\tau_{2}=10$ \\
& $\tau_{2}=70$ & $\tau_{2}=10$ & $\tau_{2}=10$ & $\tau_{3}=100$ \\
& $\tau_{3}=270$ & $\tau_{3}=100$ & $\tau_{3}=80$ & $\tau_{4}=700$ \\
\hline
\end{tabular}

\section{Ligaments}

Spinal ligaments were added to each motion segment and their insertion points and cross-sectional areas (CSA) were taken from other studies (Goel et al., 1995; Shirazi-Adl et al., 1986; Lu et al., 1998; White and Panjabi, 1990; Pintar et al., 1992) (Figure 7.2a). Tensile elastic modulus was assumed to be the same for all ligaments due to lack of material property data on the individual ligaments (Shirazi-Adl et al., 1986). The elastic modulus was set as $0.5 \mathrm{MPa}$ and $70 \mathrm{MPa}$ in the toe region and linear region, respectively, based on previous FEA studies and cadaver measurements (Neumann et al., 1992; Pintar et al., 1992; Shirazi-Adl et al., 1986; Lu et al., 1998; Goel et al., 1995). Nonlinear stress-strain curve of spinal ligaments measured on cadaver specimens (Pintar et al., 1992; Chazal et al., 1985; Panjabi et al., 1982; Myklebust et al., 1988; Adams et al., 1980) showed strong variability depending on the degeneration status and geometry of the samples and preparation setups (Zander et al., 2004). 
Using the cadaver study results directly in FE model without modification of the curve could result in considerable error. FE models assumed full upright standing posture as an initial posture of lumbar flexion motion (Zander et al., 2001; Shirazi-Adl, 1994; Goel et al., 1993; Wang et al., 2000) while cadaver samples might not be in the full upright standing posture when they were being stretched. In addition, the location of ligament attachment points and initial length of ligaments might be different between the FE models and the cadaver samples and these could generate a large discrepancy when estimating ligament strain (Cholewicki and McGill, 1992). In vivo measurements of distances between adjacent vertebral bodies in lumbar flexion motion from upright standing to fully flexed posture suggested greater strain of spinal ligaments than the measurements by cadaver samples (Pearcy and Tibrewal, 1984; Cholewicki and McGill, 1992). Therefore, the FE model of this study used a longer toeregion than in vitro measurements as shown in the nonlinear stress-strain curves in Figure 7.3. Based on the failure stress of in vitro observations (Chazal et al, 1985; Pintar et al., 1992), each ligament curve was defined to have its maximum stress less than $20 \mathrm{MPa}$ throughout all FE simulations of this study.

A reduced relaxation function by Yahia et al. (1991) was used for developing the Prony series for the shear modulus. The relationship was described by the function,

$G(t)=0.95-0.044 \ln t$

and it was transformed to the Prony series of shear modulus $G(t)$, 
$G(t)=G_{0}\left(1-\sum_{i=1}^{n} G_{i}\left(1-e^{-t / \tau_{i}}\right)\right)$

Where $t=$ time, $G_{0}$ is the instantaneous elastic modulus at $t=0, G_{i}$ is the Prony series weighing factor, and $\tau_{i}$ is the relaxation time constant.

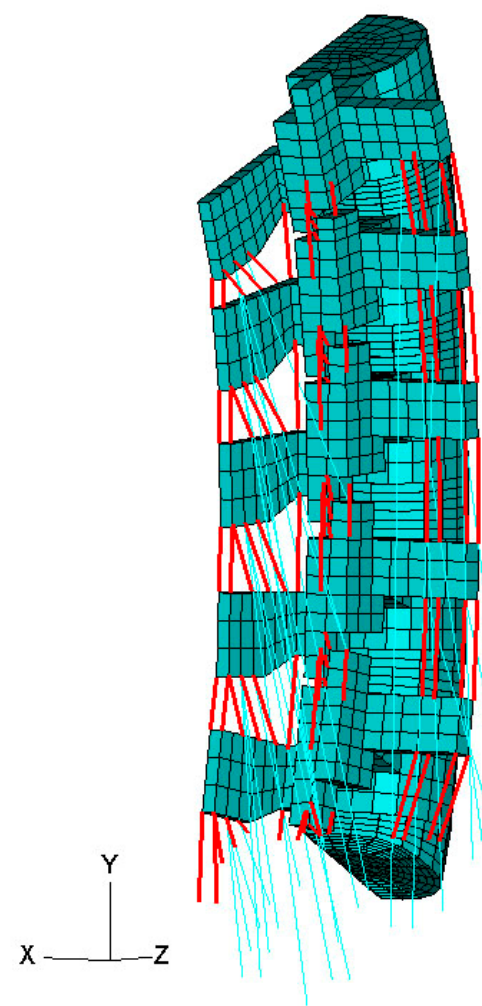

(a)

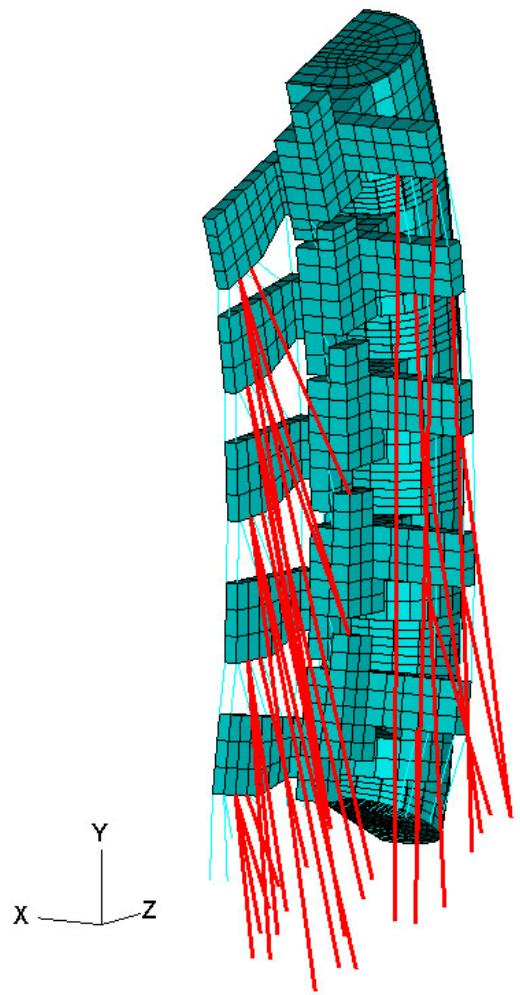

(b)

Figure 7.2. Elements for (a) ligaments and (b) muscles (shown in red). 


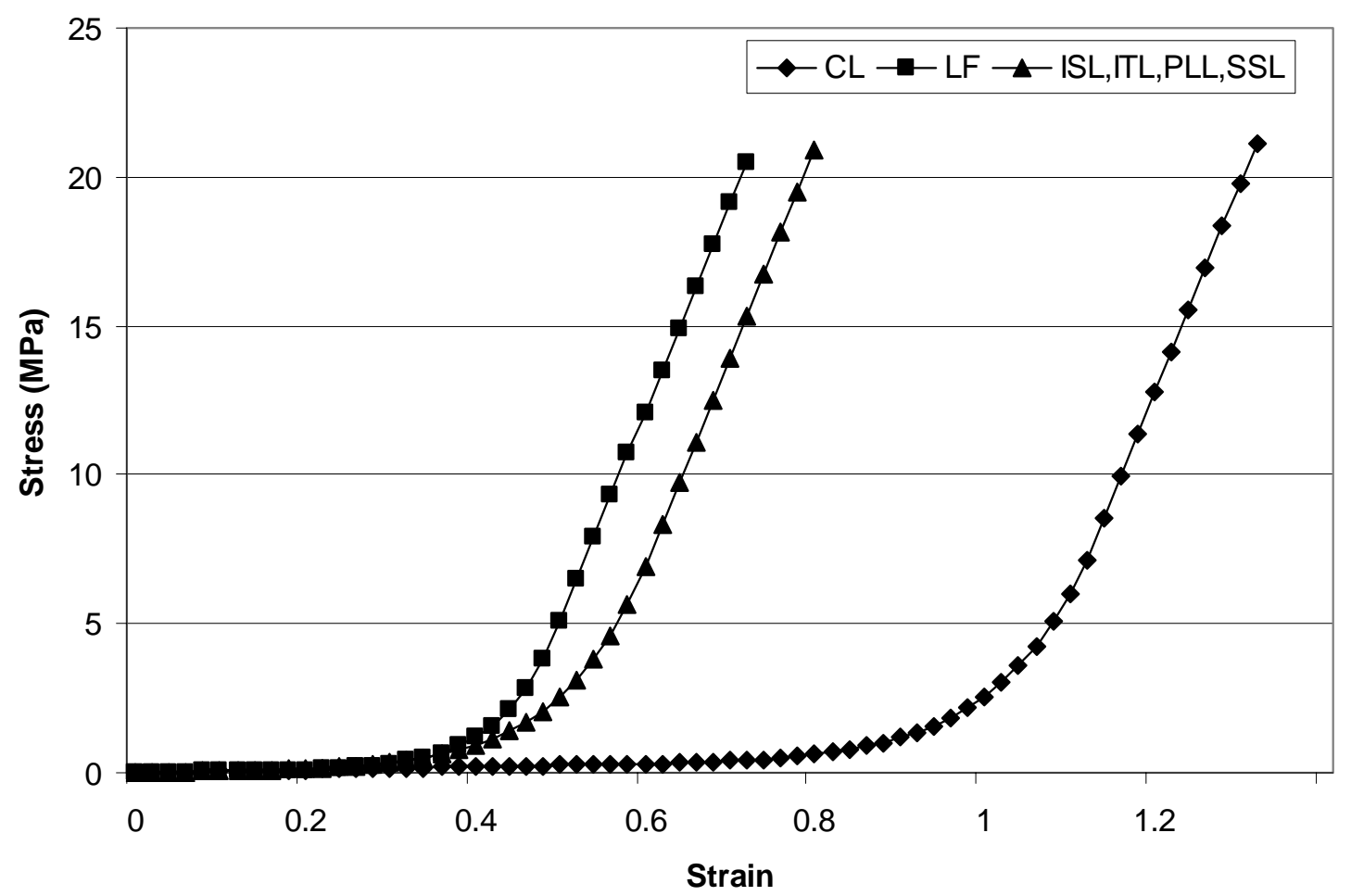

Figure 7.3. Nonlinear stress-strain curves of spinal ligaments.

\section{$\underline{\text { Extensor muscles }}$}

Major lumbar extensor muscles were included and passive stretch of the muscle elements was considered in the model. Active force by the lumbar extensor muscles was also assessed by computing reaction forces on the muscle attachment points during weight holding task simulations. Insertion and origin points, and physiological cross-sectional area (PCSA) of lumbar erector spinae and multifidus muscles were determined based on measurements by Bogduk et al. (1992) (Figure 7.2b). Muscles generate passive tension force in stretching and the passive force increases in an exponential fashion as the muscle is stretched beyond its unstressed length (Gareis et al., 1992). Because of lack of information on the stress-strain relationship of human back extensor muscles in longitudinal tension load, 
it was assumed that the maximum tensile passive force at full flexion would be greater than the maximum active force, but less than twice the maximum active force of the muscle, based on in vivo experimental measurements of other muscles (Gareis et al., 1992; Bobbert et al., 1990). In vivo, the maximum active force per unit area of the erector spinae for a human has been reported as $0.48 \mathrm{~N} / \mathrm{mm}^{2}(0.48 \mathrm{MPa})$ (Reid and Costigan, 1987) so the maximum tensile stress at full flexion was set to $0.7 \mathrm{MPa}$ (greater than $0.48 \mathrm{MPa}$ but less than $0.48 * 2$ MPa). Ultimate tensile strength of human cadaver muscles of people 20 to 39 years of age range from 0.1 MPa to 0.3 MPa (Yamada and Evans, 1970) but, with consideration of the rapid postmortem decrease in the ultimate strength of muscle tissue, the maximum stress of 0.7 MPa seemed a reasonable choice. The reduced stress-relaxation relationship of sheep tendon by Sarver et al. (2003) was used for the viscoelastic material properties of muscle elements because the stress-relaxation patterns of in vivo muscle-tendon units resemble the stress-relaxation of tendon samples (Kubo et al., 2002; Magnusson et al., 2000) (Figure 7.4). 


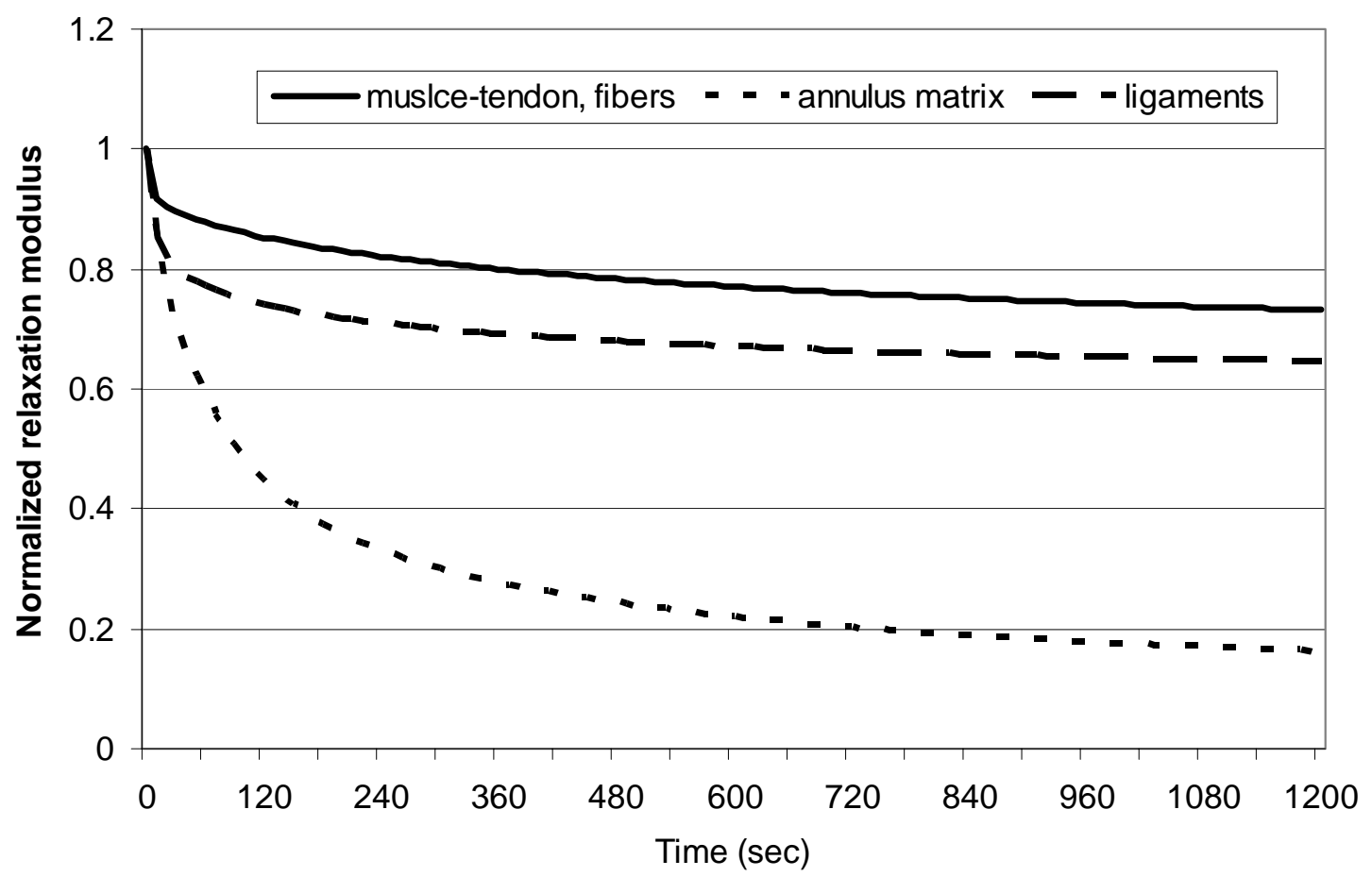

Figure 7.4. Normailized relaxation functions of lumbar spine tissues in the model $\left(G(t) / G_{0}\right.$ for fibers and ligaments, $K(t) / K_{0}$ for annulus matrix).

\subsubsection{Loading}

The model simulated a 10-minute full lumbar flexion (stooped posture) followed by a 10-minute upright standing posture with static weight holding postures every 2.5 minutes. A reference node (Figure 7.5) was defined at the center of gravity of torso-arms at fully flexed posture (Chaffin et al., 1999) and this node was connected to the top surface of the L1 vertebral body with a 'rigid body' interaction so the motion of the reference node governed the motion of the L1 top surface. This point was located $390 \mathrm{~mm}$ above and $25 \mathrm{~mm}$ anterior to the center of the L5/S1 disc in the sagittal plane at the upright standing posture. The bottom surface of L5/S1 disc and the end nodes of ligament and muscle elements on S1 were fixed in all directions (Figure 7.6). 


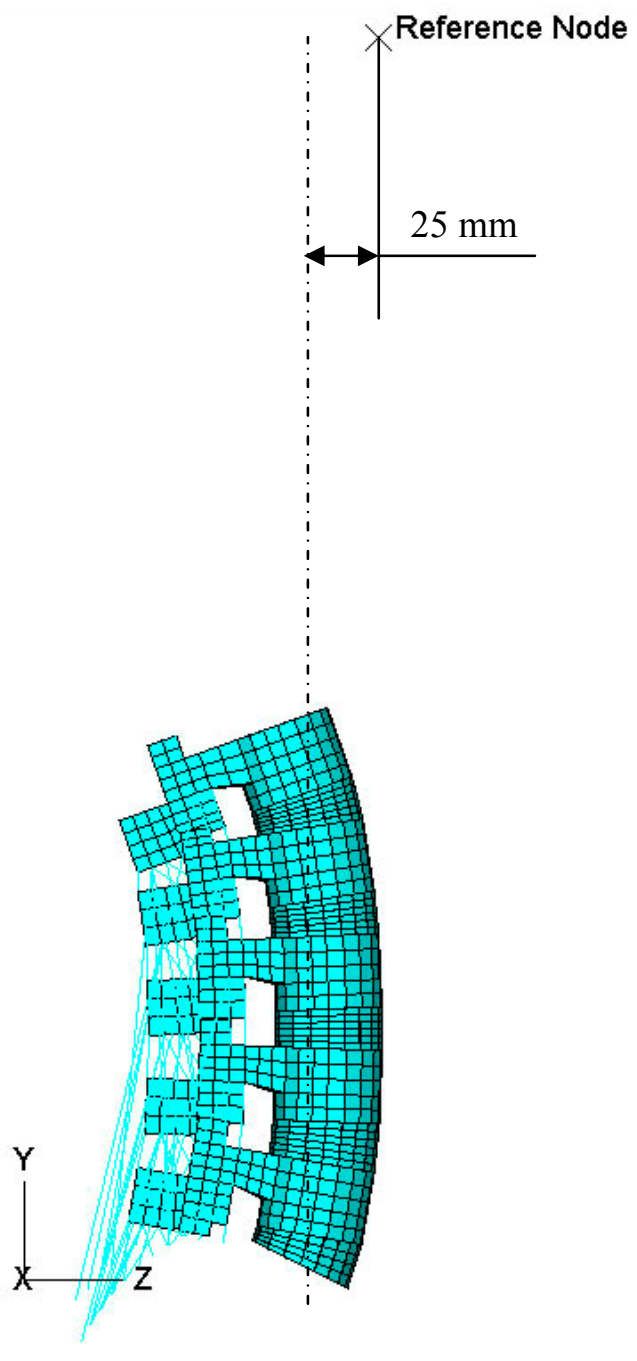

(a)

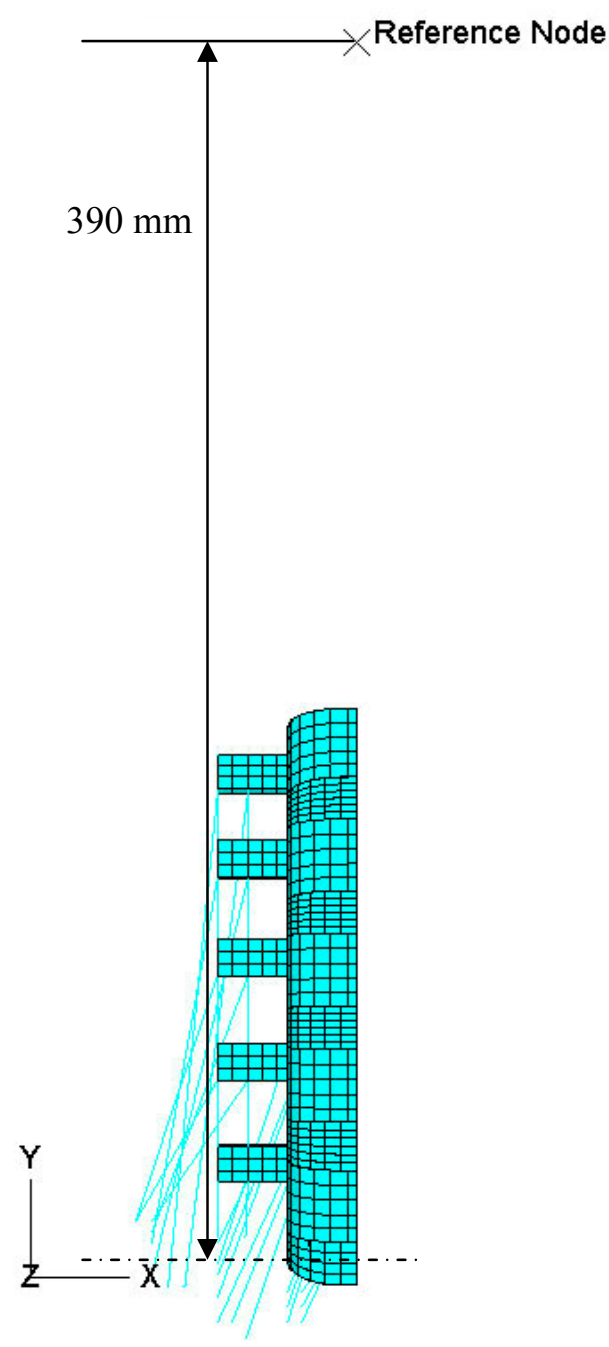

(b)

Figure 7.5. Reference node location (a) side view, (b) front view. 


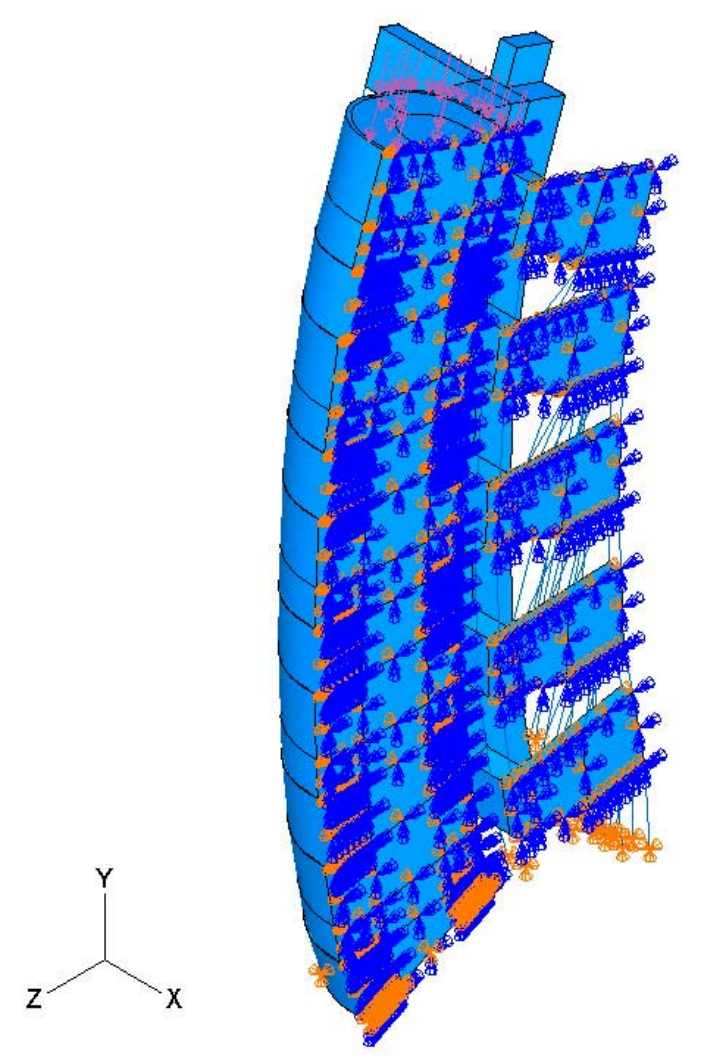

Figure 7.6. Boundary conditions for 'standing' load step.

\section{Standing posture}

Since the intervertebral discs lose their thickness under a pure compression load (Burn et al., 1984; Li et al., 1995), the FE model assumed a 30-minute quiet upright standing posture at the beginning of the simulation. For the upper body weight on the lumbar spine, a static distributed load of $400 \mathrm{~N}$ (55\% of the $75 \mathrm{~kg}$ body weight, Chaffin et al., (1999)) was applied to the top surface of the L1 vertebral body for 30 minutes. Anterior-posterior directional displacement of the vertebral bodies was constrained and vertical and lateral directional displacements were allowed. Table 7.5 describes the progression of simulated tasks (loading steps) with applied boundary conditions. 
Table 7.5. Loading steps (progression of simulated tasks) and boundary conditions (BCs).

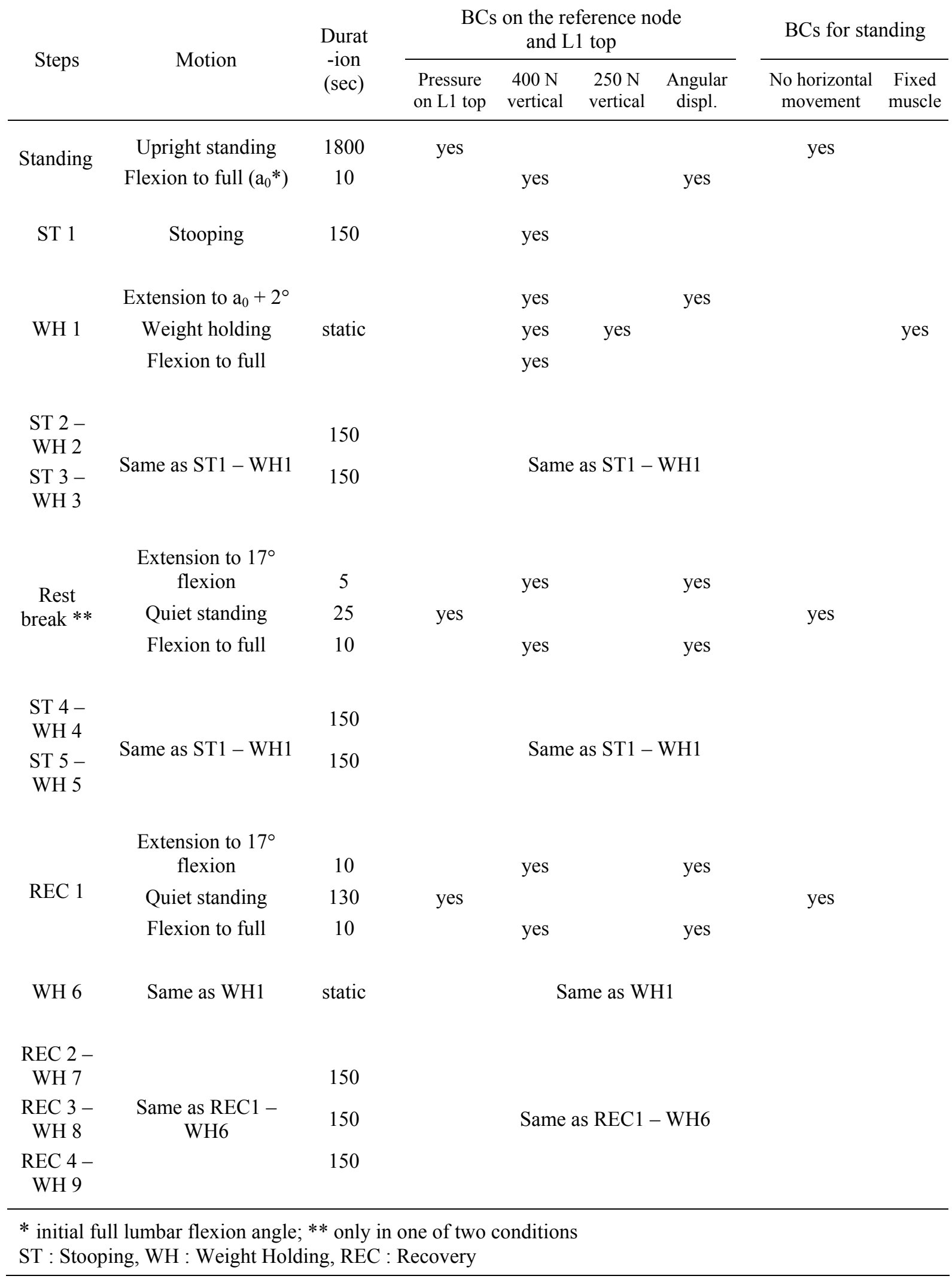




\section{Stooped posture}

Lumbar sagittal flexion was controlled by an angular displacement and concentrated force on the reference node (Figure 7.5). The reference node was rotated forward (anterior direction) around the L5/S1 disc in a period of 10 seconds and a $400 \mathrm{~N}$ vertical force was applied on the reference node as an upper body weight. At the end of lumbar flexion, the angular displacement constraint of the reference node was released so the rotation of the reference node was determined by the balance between the upper body weight and the passive resistance force of the spine, which changed over the duration of stooping due to the viscoelastic properties of the lumbar spine tissues. This creep loading condition continued for 10 minutes with intervening steps for simulating weight holding tasks and a rest break. The lumbar flexion angle at 0 minute of this creep load step was defined as the initial lumbar flexion angle.

\section{Weight holding}

Every 2.5 minutes from the beginning of creep loading, the reference node was rotated backwards to $2^{\circ}$ over the initial flexion angle, and the origin nodes of back extensor muscles were fixed in all direction to simulate active forces of the lumbar extensors. Then, an additional $250 \mathrm{~N}$ vertical force was added to the reference node as an external weight on the hands, and the angular displacement of the reference node was released so the vertical load on the reference node was supported by passive resistance of the lumbar spine and the reaction forces on the muscle origin nodes. Once the lumbar spine reached static equilibrium ( 1 second), the constraints on the muscle nodes and the additional $250 \mathrm{~N}$ on the reference 
node were removed and the lumbar spine returned to the stooped posture or upright standing posture.

\section{$\underline{\text { Rest break }}$}

In a simulation with a rest break (w/rest condition), a 30 -second rest break in the upright standing posture was added in the middle of the static full flexion period. At the end of the second weight holding step, the lumbar spine was extended to a $17^{\circ}$ lumbar flexion angle by angular displacement of the reference node. Since a 'tensile-only' option was not available with nonlinear viscoelastic truss elements in ABAQUS v6.4, some ligament and muscle elements could be under compression load when the lumbar spine was extended after prolonged stooping, as illustrated in Figure 7.7. To prevent the compression load on the ligament and muscle elements, the extension was stopped at $17^{\circ}$ lumbar flexion at which the stress of most ligament and muscle elements are close to zero so the minimal amount of compression load was present in those elements. At this mildly flexed posture, the same boundary conditions as the 'standing' step were applied and held for 30 seconds. The lumbar spine was then flexed again and the stooped posture loading condition continued. 


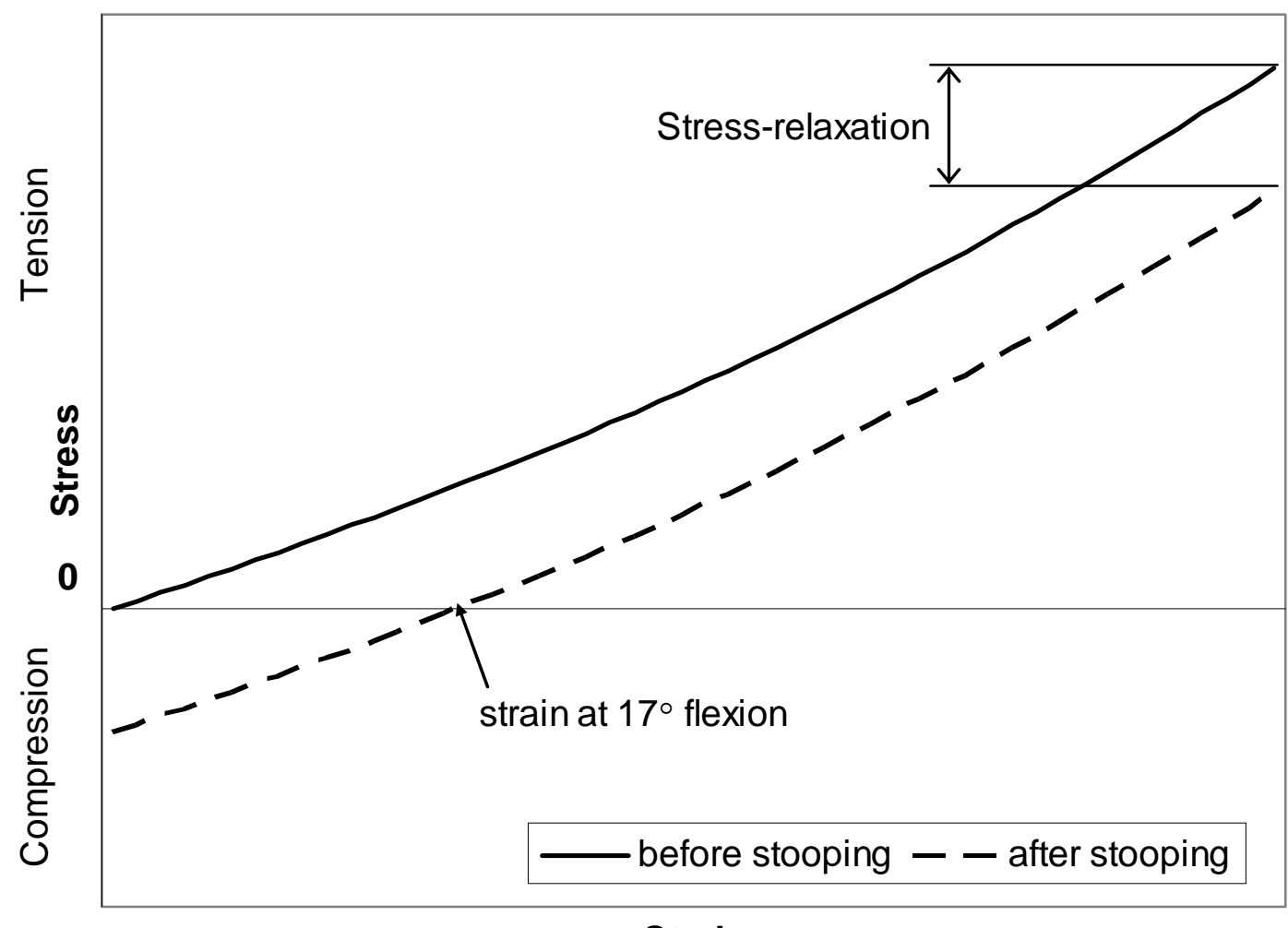

Strain

Figure 7.7. A conceptual illustration of stress-strain curve before and after stooping.

\section{$\underline{\text { Recovery standing }}$}

Immediately after the 10 minutes of stooping, the lumbar spine was extended to the mildly flexed posture and sustained for 2.5 minutes to simulate recovery standing posture. The spine was then flexed again to its full flexion angle and the simulation of the weight holding task followed. The 10-minute recovery simulation consisted of four repetitions of recovery-full flexion-weight holding steps. 


\subsection{Results}

\subsubsection{Vertical Displacement under Axial Compression Load}

The lumbar spine model was subjected to a $400 \mathrm{~N}$ vertical compression load on its top surface of the L1 segment (Figure 7.6). An instantaneous downward vertical displacement of the L1 top surface was $1.83 \mathrm{~mm}$, and the vertical displacement increased to $2.71 \mathrm{~mm}$ in 30 minutes of 'standing' loading, indicating the creep deformation of the intervertebral discs. These results were compared to the three-parameter-solid models that were developed from in vitro cadaver sample measurements (Burns et al., 1984; Li et al., 1995) (Table 7.6). The current FE model predicted a similar behavior to the in vitro observations.

In addition, the predicted full flexion angle data in 10 minutes of stooping was compared with and without the 'standing' loading steps to examine the effects of 'standing' loading prior to stooping with results shown in Figure 7.8. Stooping without the preload step produced greater instantaneous full flexion angle and creep deformation than the simulation with the preload step. 
Table 7.6. Vertical displacement of the lumbar spine in axial compression load.

\begin{tabular}{cccc}
\hline Time (min) & FE model & Burns et al.,1984 & Li et al.,1995 \\
\hline 0 & $1.83 \mathrm{~mm}$ & 1.68 & 2.20 \\
30 & $2.71 \mathrm{~mm}$ & 2.79 & 2.90 \\
\hline
\end{tabular}

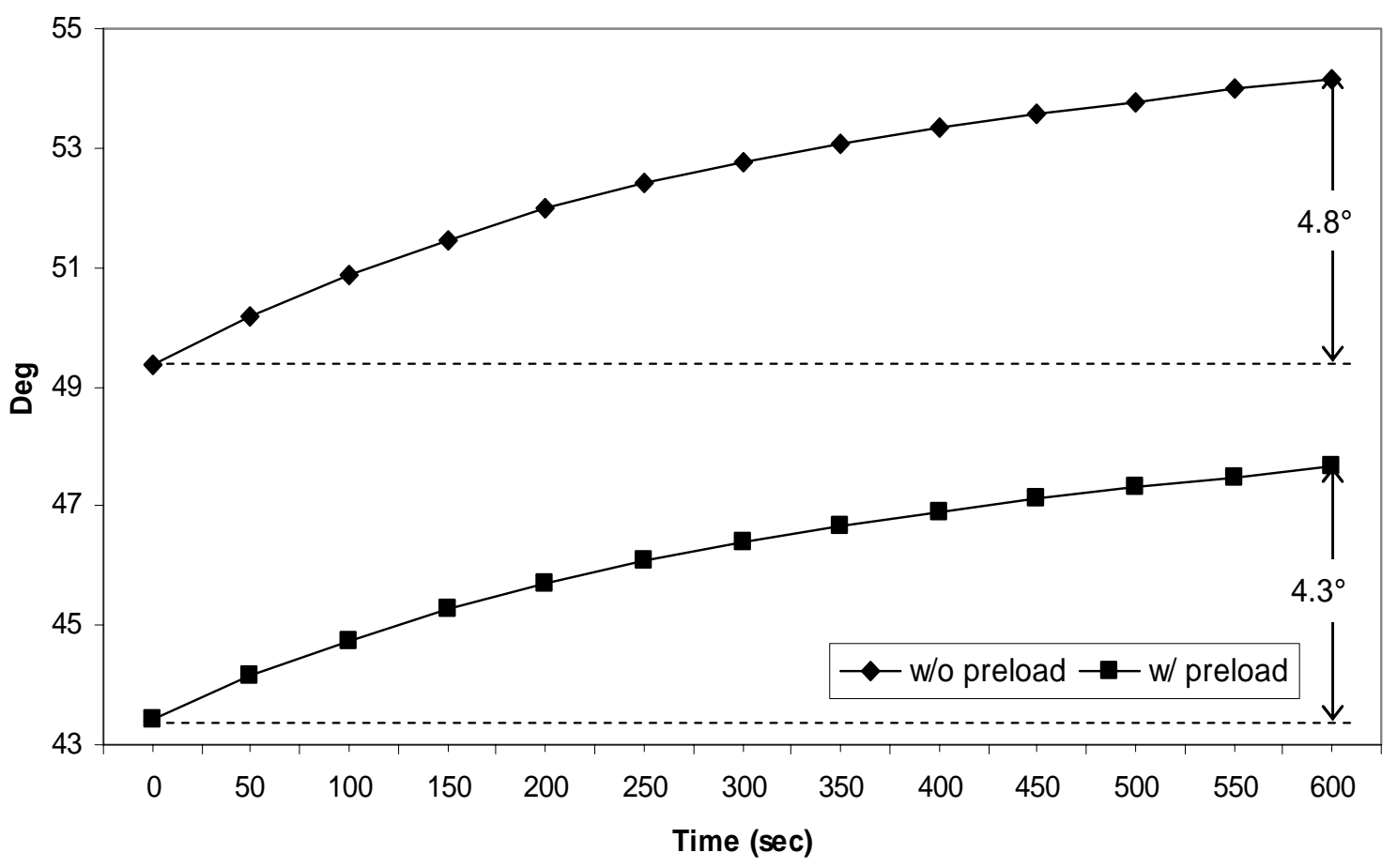

Figure 7.8. Predicted full flexion angle with and without a preload. 


\subsubsection{Full Lumbar Flexion Angle in Stooping and Recovery}

Full lumbar flexion angle was defined by the L1 top surface angle in the sagittal plane. The angle at upright standing was set to zero. In a simulation without a rest break, the full lumbar flexion angle increased continuously from $43.4^{\circ}$ to $47.7^{\circ}$ during stooping and decreased to $45.1^{\circ}$ during recovery. The rest break in the middle of stooping lessened the cumulative creep deformation of the flexion angle after the break. Predicted results were compared with in vivo observations (Figure 7.9). McGill and Brown (1992) measured a creep deformation of the full flexion angle during a 20-minute continuous stooped posture. The data from 47 human subjects was fitted by

$d(t)=d_{f}\left(1-e^{-t / T}\right)$

where, $d(t)=$ creep flexion in degree; $d_{f}=$ maximum creep-flexion angle $=6.2^{\circ} ; t=$ time in minute: $T=$ first-order time constant $=9.4$ minutes.

The in vivo experiment of current study (Chapter 6) observed the creep of the full flexion angle with identical procedures to the current FE simulation. Since only the timedependent change of the angle is relevant to the viscoelastic behavior of the lumbar spine, the experimental data sets were transposed along $\mathrm{Y}$-axis so the angle of in vivo data and $\mathrm{FE}$ results could have a common value at 0 minute.

The quantitative comparison between the FE model results and the in vivo data sets was performed by examining the Pearson's correlation coefficients and mean absolute errors. The Pearson's correlation coefficients assessed the strength of a linear relationship between 
the pairs (Table 7.7), and the mean absolute error measured the overall error of the FE model data relative to in vivo observations (Table 7.8).

Both the quantitative and graphical comparisons (Figure 7.9) indicated good agreement between the predicted full flexion angle and the in vivo observations throughout both the stooping and the recovery period, while the comparison of w/rest condition showed a less strong influence of the rest break on the FE predicted angle than on the in vivo observations.

Table 7.7. Pearson's correlation coefficients between the FE predicted angle and the in vivo observed angle.

\begin{tabular}{ccc}
\hline & FE vs. In vivo experiment (Ch. 6) & FE vs. McGill and Brown (1992) \\
\hline no-rest & 0.9958 & 0.9955 \\
w/rest & 0.9798 & - \\
\hline
\end{tabular}

Table 7.8. Mean absolute errors between the FE predicted angle and the in vivo observed angle. FE vs. In vivo experiment (Ch. 6) FE vs. McGill and Brown (1992)

\begin{tabular}{rcc}
\hline no-rest & 0.11 & 0.24 \\
w/rest & 0.39 & - \\
\hline
\end{tabular}




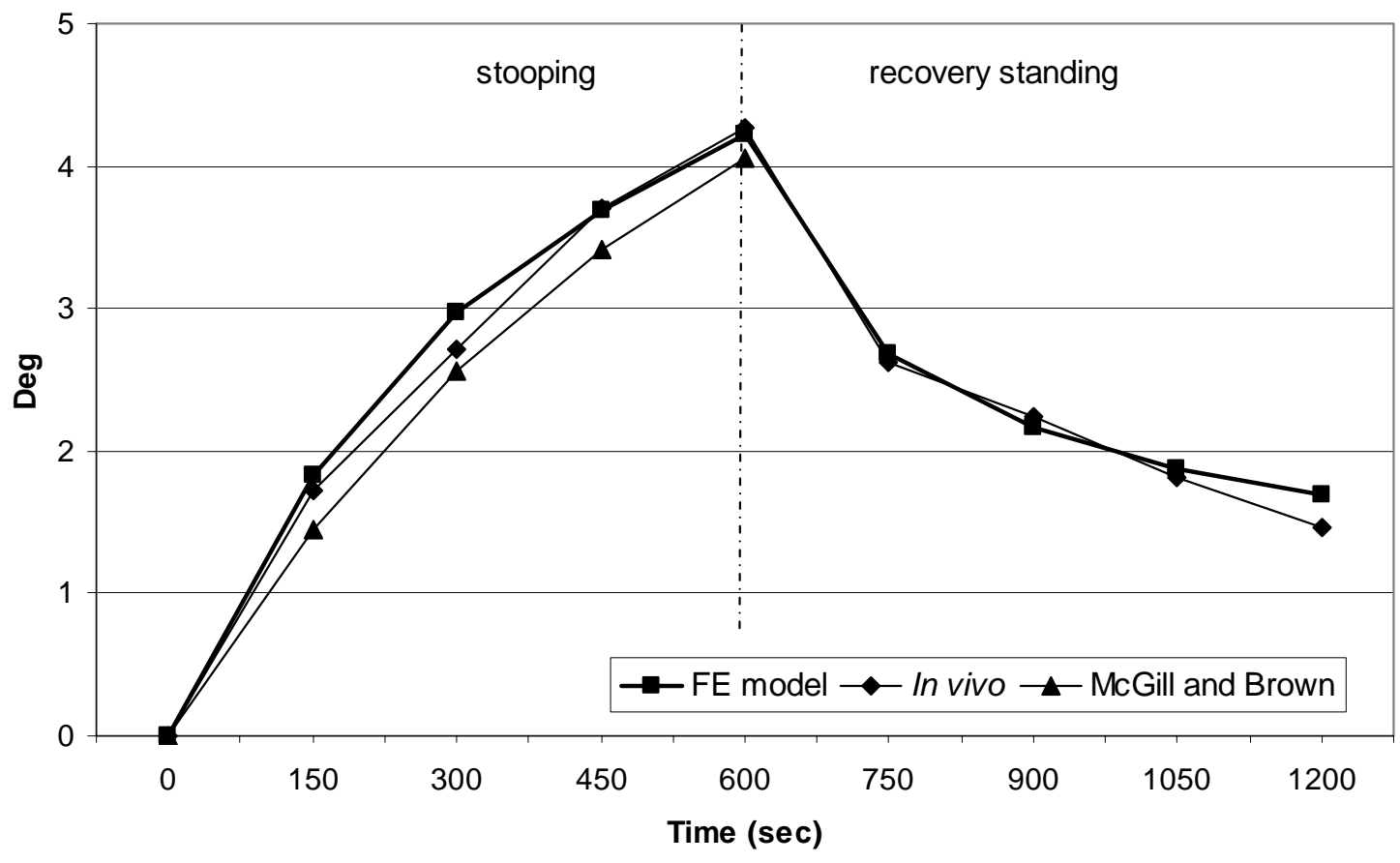

(a)

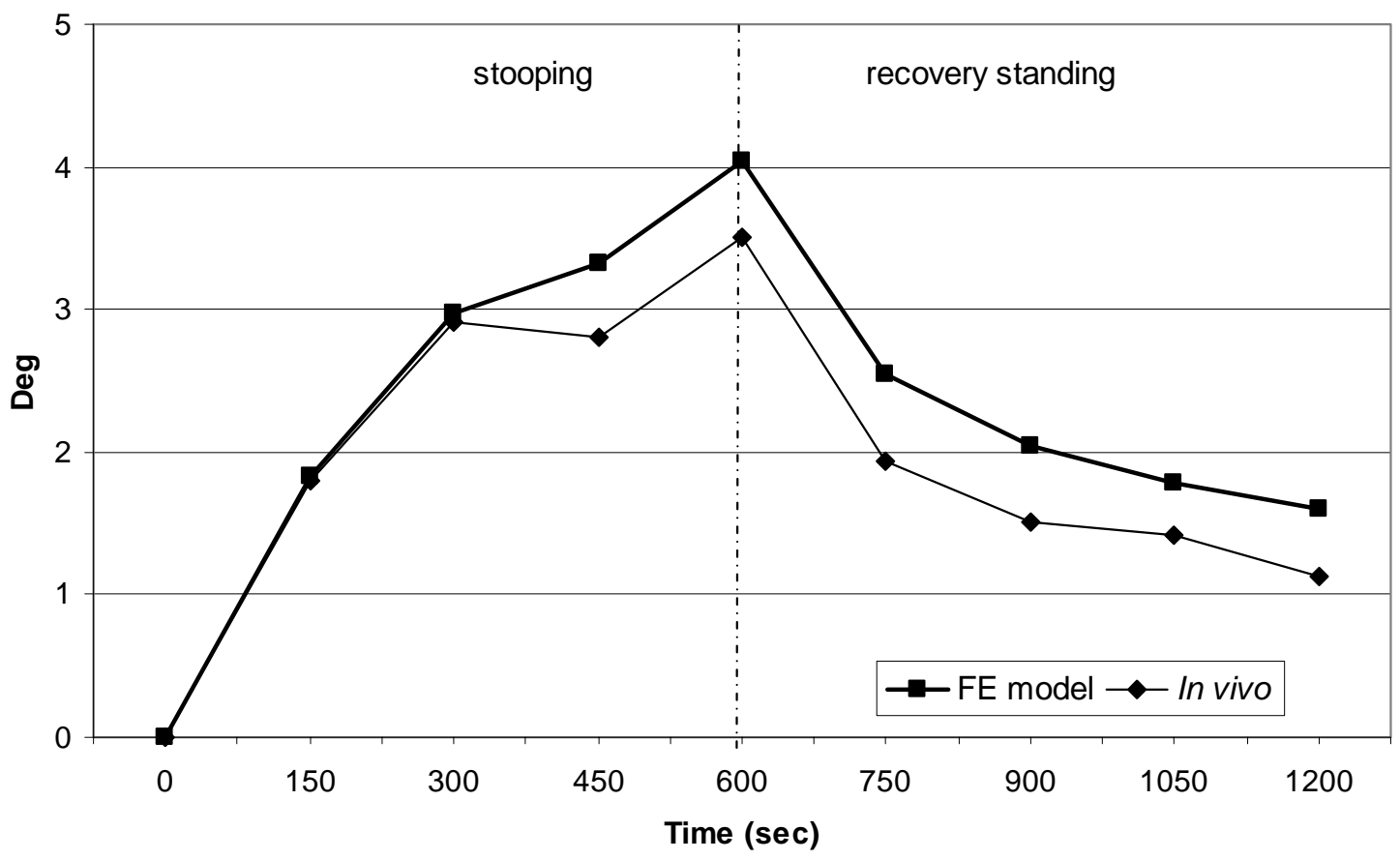

(b)

Figure 7.9. Full lumbar flexion angle during a 10-minute stooping and a 10-minute recovery. (a) no-rest condition, (b) w/rest condition (30-sec rest break at 300 sec. Not included in the duration of the stooping) 


\subsubsection{Reaction Force at Weight Holding Task}

Square root of sum of squares (SRSS) of $\mathrm{x}, \mathrm{y}$, and $\mathrm{z}$ directional reaction forces were obtained from all muscle origin nodes at each weight holding step, and the SRSS reaction forces were averaged within each muscle group. The predicted reaction forces of all muscle groups were averaged because no apparent difference in the reaction force between individual muscle groups was observed. For the validation of the model, the predicted forces were compared with in vivo muscle EMG observations collected with the same experimental design (Chapter 6) (Figure 7.10). Because of different orientation of force vectors between the SRSS reaction force in the FE model and the muscle contraction force in the experimental study, the two data sets were normalized relative to their initial values at time 0 , and the ratios of the two were compared. The Pearson's correlation coefficients and the mean absolute errors between the FE simulation results and the in vivo EMG data are listed in Table 7.9 and 7.10, respectively.

Table 7.9. Pearson's correlation coefficients between the FE predicted force and the in vivo EMG.

FE vs. In vivo Multifidus

FE vs. In vivo Erector spinae

$\begin{array}{lll}\text { no-rest } & 0.9190 & 0.9534 \\ \text { w/rest } & 0.9239 & 0.9100\end{array}$

Table 7.10. Mean absolute errors between the FE predicted force and the in vivo EMG.

\begin{tabular}{rcc} 
& FE vs. In vivo Multifidus & FE vs. In vivo Erector spinae \\
\hline no-rest & 0.0847 & 0.0347 \\
w/rest & 0.0747 & 0.0610 \\
\hline
\end{tabular}




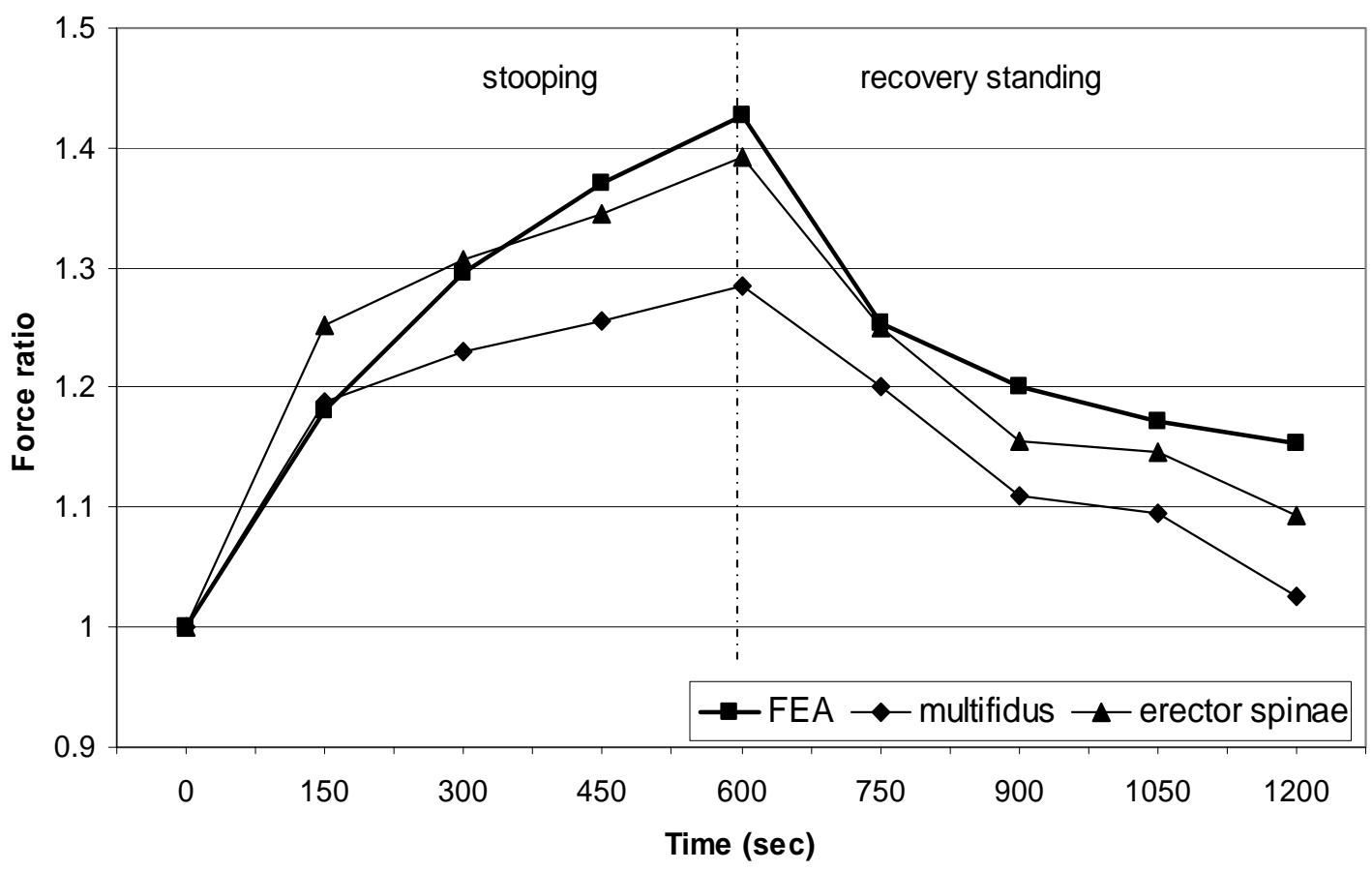

(a)

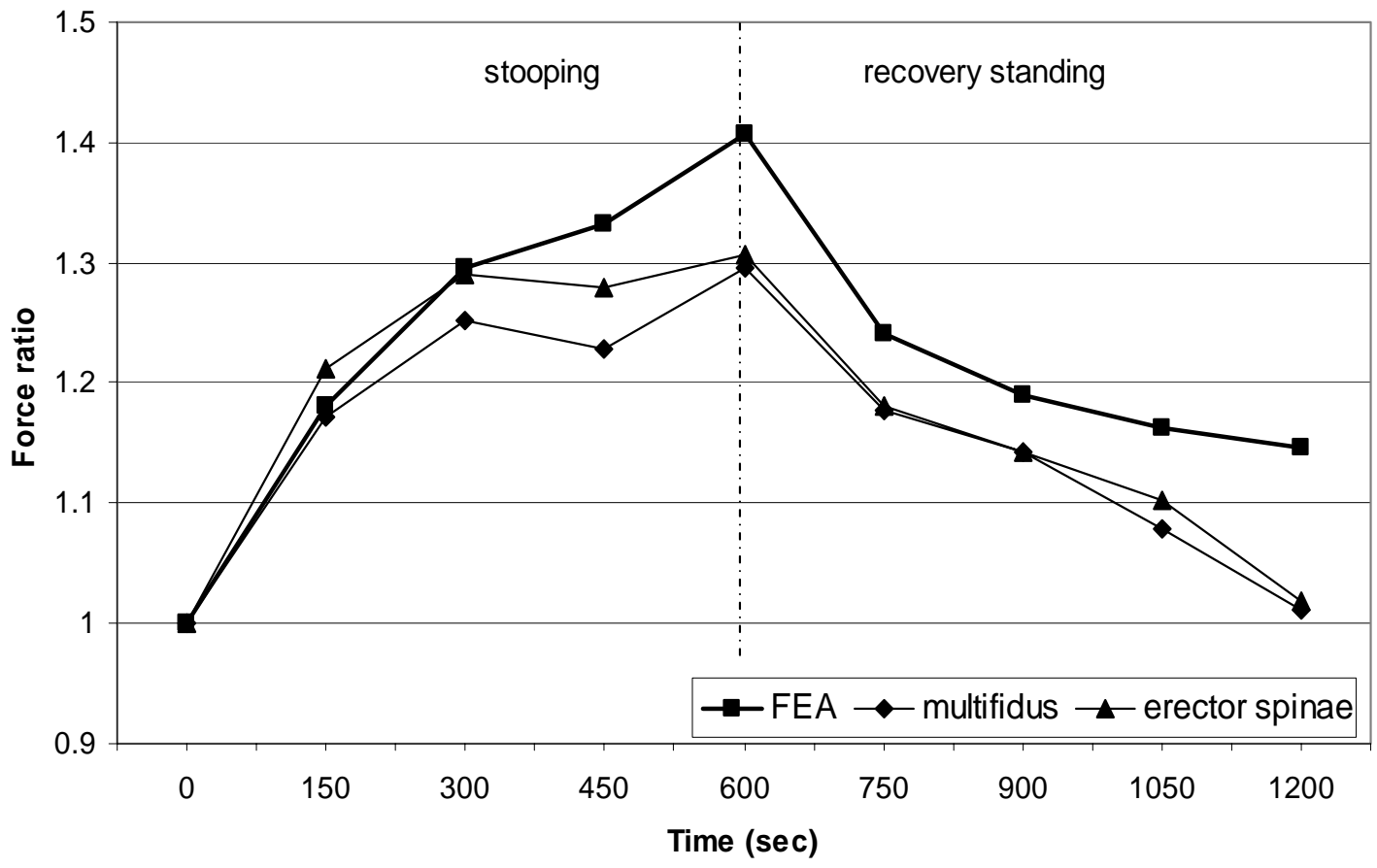

(b)

Figure 7.10. Muscle force in weight holding task (a) no-rest condition, (b) w/rest condition. 


\subsubsection{Hydrostatic Pressure of the Nucleus Pulposus}

A time-dependent change of the hydrostatic pressure in the nucleus pulposus at full flexion posture was examined (Figure 7.11). The average pressure of each nucleus was computed before and after 30 minutes of standing, and before and after 10 minutes of stooping simulation (Figure 7.12). The predicted disc pressure in standing (400 N load) ranged between 0.3 and $0.4 \mathrm{MPa}$ and this agreed well with in vitro measurements by McNally and Adams (1992). In their study with cadaver samples, the horizontal and vertical stress in the middle of L2/L3 disc nucleus under $500 \mathrm{~N}$ compressive force at zero flexion were observed as $0.5-0.55 \mathrm{MPa}$ for non-degenerated young discs and 0.45-0.5 MPa for degenerated old discs.

The predicted pressure increased up to 5.4 times by full lumbar flexion and a following 10-minute creep loading at the full flexed posture produced more pressure on the nucleus. The increased pressure in the nucleus by lumbar flexion was also observed in the experimental study (McNally and Adams, 1992) but a direct comparison with the FE model results was not performed because of different experimental setups. 

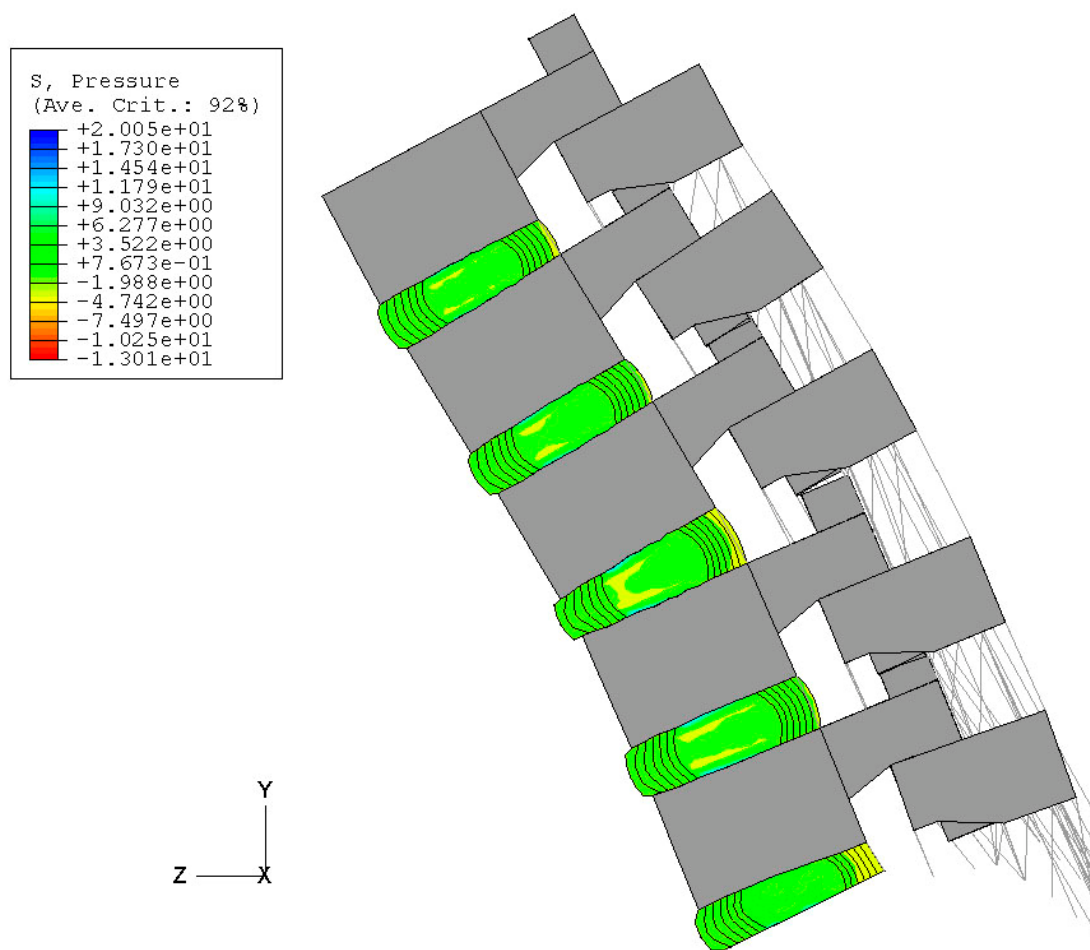

Figure 7.11. Hydrostatic pressure (MPa) of intervertebral discs after 10-minute stooping.

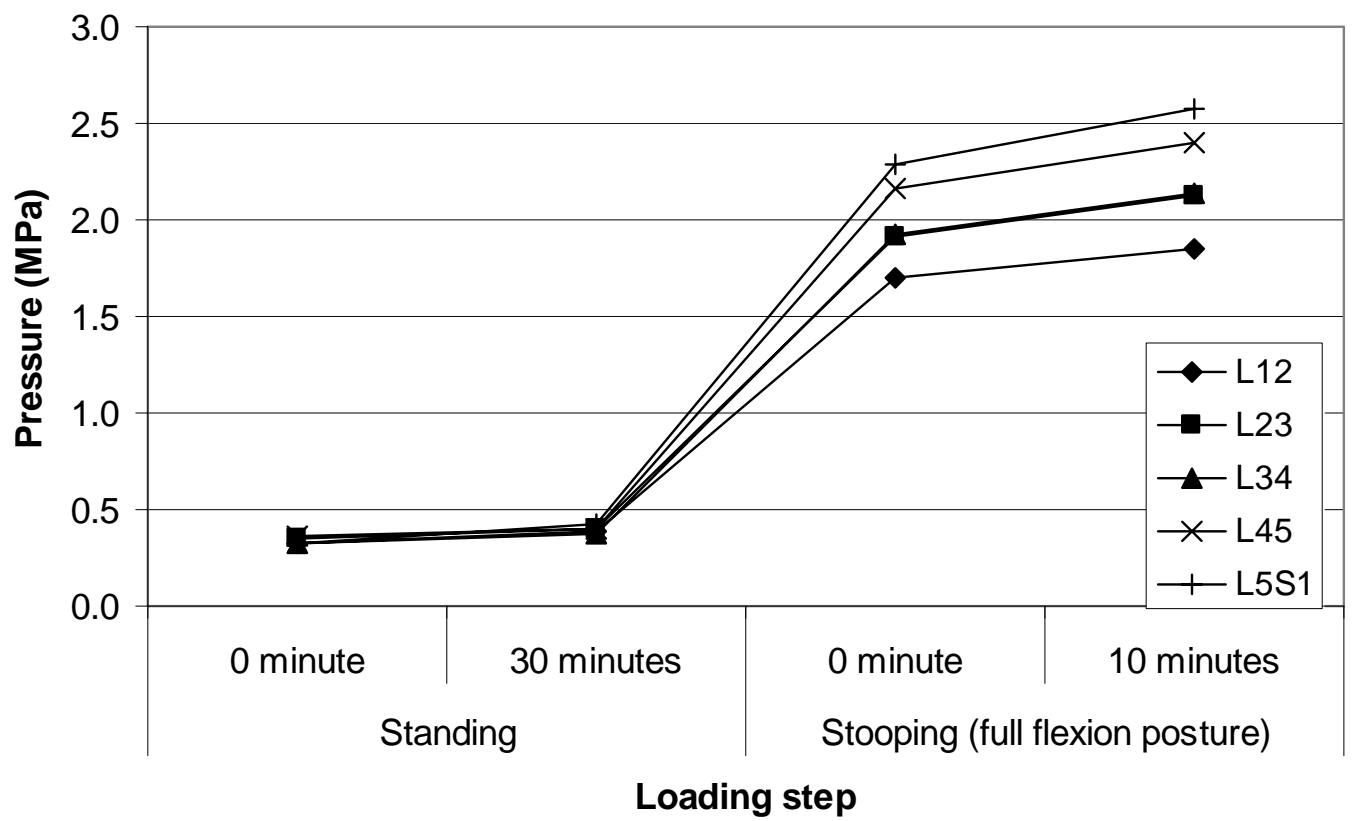

Figure 7.12. Nucleus pressure at each loading step as predicted with FE model. 


\subsection{Discussion}

The FE model simulated a prolonged stooping and upright standing posture with cyclic weight holding tasks, and the predicted results were compared with experimental observations to validate the model.

The current FE model included a quiet standing loading step as a preload on intervertebral discs prior to lumbar flexion and it produced different results than the simulation without the preload step. The disc components such as annulus matrix and fibers generate more resistant force as they are deformed. The preload causes the deformation of the disc and generates pre-stress in the disc. Because of this pre-stress, the intervertebral disc with the preload could require less deformation than the intact disc without the preload to produce the same amount of resistant moment, and it might result in less full lumbar flexion angle in the simulation with the preload.

Many previous lumbar spine FE models did not include the pre-load effect on prediction of the mechanical behaviors in lumbar flexion (Shirazi-Adl et al., 1986; ShiraziAdl, 1994; Zander et al., 2001). A study by Wang et al. (2000) added a 30-second $600 \mathrm{~N}$ compression load step prior to flexion motion as a preload in neutral upright posture, but their duration of the preload might not have been enough to simulate a realistic in vivo situation. Results of the current FE model showed influences of the preload on the mechanical behaviors of the lumbar spine flexion, suggesting the preload step needs to be present prior to lumbar flexion simulation. Even though the importance of the preload step is not questionable, it needs to be noted that the amount of duration and load need to be defined with careful consideration of in vivo diurnal variation of intervertebral disc height and pressure. 
Comparisons between the FE model results and the in vivo observations showed high correlation coefficients $(\mathrm{R}>0.9)$ with relatively small mean absolute errors, indicating good agreement in terms of time-dependent changes in the dependent variables. The discrepancy observed after a rest break could be attributed to two limitations in the FE model. Different from in vivo experiments, the lumbar spine model was not fully extended to neutral upright standing posture in the simulations of the rest break and recovery posture to prevent compression load on ligament and muscle elements. In this mildly flexed posture $\left(17^{\circ}\right)$, there could be more pressure on intervertebral discs than in the upright standing posture and some ligaments or muscles might be still stretched, delaying their recovery. This might result in the less significant effect of the rest break in the FE model compared to in vivo data. Another limitation was from an assumption that viscoelastic strength recovery and stressrelaxation curve of spinal tissues are inversely related in the FE model. Due to lack of information on the viscoelastic recovery pattern of lumbar spine tissues and the limitation of the FEA software, the FE model assumed that the elastic modulus increases along the inverse path of the stress-relaxation curve when creep load is removed or reduced. Based on the comparison between in vivo observations and predicted results, it was assumed that in vivo lumbar spine tissues recover their strength faster than the FE model predicted, in the earlier stage of the recovery period.

The calculated reaction force increased approximately $40 \%$ in stooping and more than one third of this increase was not recovered after a 10-minute recovery. At the fully flexed posture, the upper body is supported simultaneously by active forces from the extensor muscles and the passive tension force by the lumbar spine tissues. Reduced strength of passive tissues due to stress-relaxation during stooping is compensated for by the increased 
activity of the extensor muscles. To support the idea of transfer of extension moment from passive tissues to active muscles, the stress of ligaments at each weight holding step was examined. Figure 7.13 shows a typical relationship of ligament stress at each weight holding step. All ligaments showed reduced stress at weight holding tasks in stooping. Together with the ligament stress change, the predicted reaction forces clearly showed this passiveactive force transfer relationship as a function of time.

In Figure 7.7, the predicted force was greater than the in vivo EMG data and the difference increased in stooping. Muscles lose their force-generating capacity and exhibit reduced activation when they are passively stretched (Fowles et al., 2000; Avela et al., 2004). The fully flexed posture is supported by passively generated forces by the extensor muscles as well as ligaments and discs, and the stretched muscles in stooping might show less EMG activity than the required force level which was predicted by the FE model simulation.

In the interpretation of the quantified results of the force prediction, it should be noted that the current FE model included only major lumbar extensors and neglected the function of other muscles and tissues such as latissimus dorsi and lumbodorsal fascia, which were known to be involved in extension moment generation and lumbar stabilization (Bogduk et al., 1998; Gracovetsky et al., 1981). Even though the contribution of those tissues in the lumbar spine may be relatively small compared to the major extensor muscles (McGill, 2002), a future FE model should consider the function of other tissues. This is also important to understand the co-activation of muscles in the lumbar spine region in the change of extensor muscle capacity after passive stretching. 


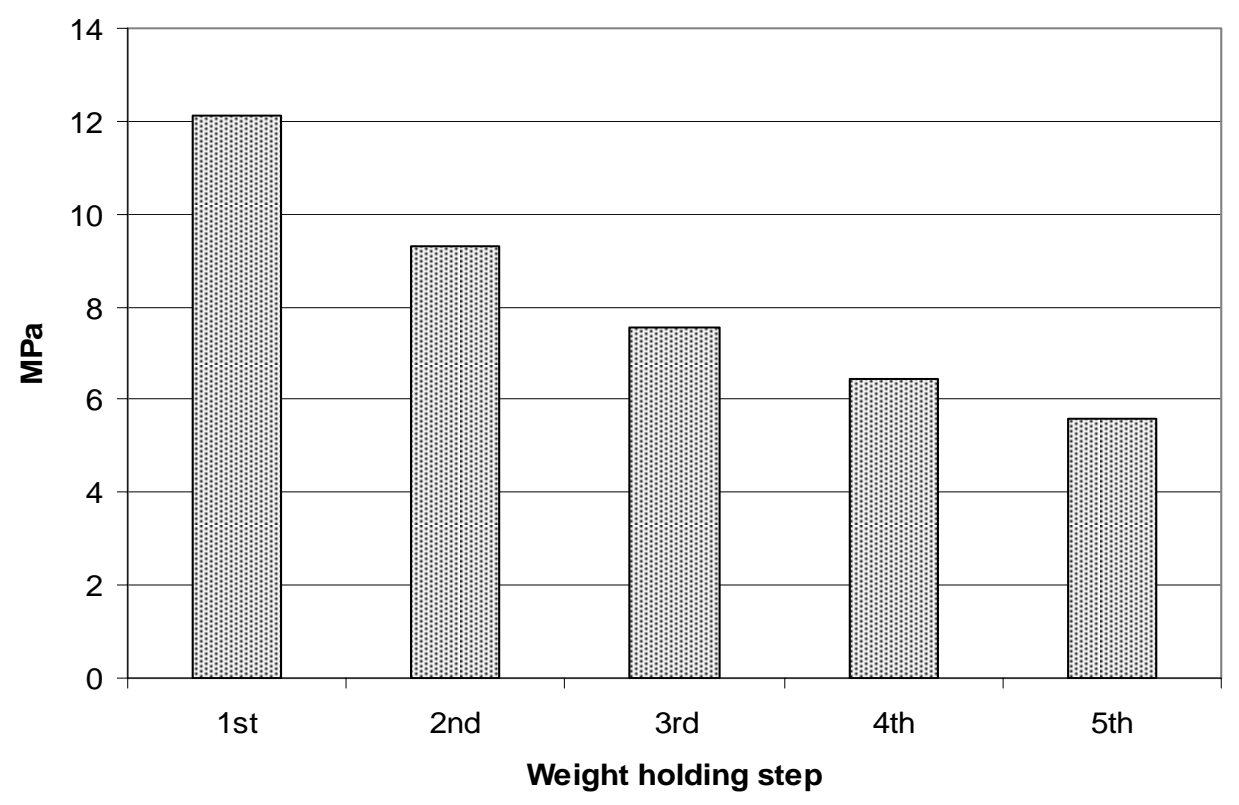

Figure 7.13. Stress of a ligament at weight holding tasks in no-rest stooping.

Despite the limitations and assumptions addressed above, the current FE model predicts mechanical behaviors in the lumbar spine during prolonged stooping, showing good correlation with experimental studies. This validation study confirmed that the model could be used to examine the injury mechanisms resulting from prolonged stooping. However, to be used as a quantitative predictor, this FE model will need further validation processes especially with more detailed geometry (initial length in standing posture) and material properties of spinal ligaments. 


\subsection{Conclusions}

A 3D FE model of a whole lumbar spine with nonlinear viscoelastic material properties was developed to predict mechanical changes in the lumbar spine during prolonged stooping and following recovery period. Creep of the full flexion angle and muscle forces at weight holding tasks were predicted by simulating full range of lumbar flexion motions with a time variance. The full lumbar flexion angle and the muscle force were predicted to increase over stooping period and decrease during recovery as a result of creep deformation and stress-relaxation of lumbar spine tissues. Validation of the model by comparing its predicted results with experimental results indicated good agreement (correlation coefficients $\mathrm{R}>0.9$ ) in terms of time-dependent mechanical behaviors in prolonged stooping. The results of this validation study confirmed the capability of this FE model as a tool to assess the physical risks of prolonged stooping in various occupational tasks. 


\section{GENERAL DISCUSSION}

The two main findings of the in vivo study (Chapter 6) were the transfer of extension moment between passive tissues and active muscles as a result of viscoelastic responses of the passive tissues during stooping and recovery, and the effects of short rest break in the middle of the stooping period. These results recognized the importance of the activity level of back extensors in an assessment of LBD risks in work-related tasks with repetitive prolonged stooped postures. As observed in Figure 6.6 and 6.7, the EMG of back extensors were less sensitive to short period of break than the full flexion angle and this might result from the nonlinear stress-strain relationship of the passive tissues (Figure 6.8). Estimating the risks of the prolonged stooping by measuring the creep of lumbar flexion might lead to the underestimation of the load on extensor muscles specifically when work tasks involve frequent rest breaks and repetitive stooping postures.

However, these different responses of the EMG and full flexion angle to the rest break were not clearly identified in the FE model simulations due to modeling limitations (Chapter 7). The FE model successfully replicated a continuous stooping posture and showed good match with in vivo observations with high correlation $(\mathrm{R}>0.9)$, but the influence of the rest break was not successfully incorporated into the model. Most workrelated tasks with prolonged stooped posture involve frequent short rest breaks or microbreaks in the middle of the tasks. The duration and frequency of the rest break during the stooping are key components that affect cumulative stress and/or fatigue on low back as well as productivity of the work. Reliable simulation for the rest break posture and load conditions is important to make use of the FE model as a risk assessment tool for LBDs. 
A future study will focus on the development of duty cycles that can reduce the risks of cumulative exposures to the prolonged stooping postures in various occupational tasks. This study will consists of in vivo simulations of the real working postures and the refining of the FE model.

The stooping posture in the current study was a two-dimensional static posture with no movement of upper and lower extremities. It is reasonable to extend this basic study to more applied research by replicating the study with less controlled postures to simulate real occupational tasks. Stooping postures in industry, such as a crop harvesting and roofing/concrete work, are more dynamic and involve frequent over flexion or mild extension of the lumbar spine, upper and lower extremity movements such as picking up an object and knee joint flexion, and asymmetric geometry or loading on the lumbar spine. The occupational tasks with these additional variables will be reproduced in the lab and subjects' time-dependent kinematics data will be captured. The observed data will be analyzed to determine independent variables such as three-dimensional kinematics of lumbar spine flexion, external weight, and movement of lower extremities, which will be used in the FE model simulations.

The FE model of the current study exhibited some limitations in predicting the effects of the rest break or recovery standing, as addressed above. The future FE model should be developed with more detailed geometry and material properties of the passive tissues. The three-dimensional geometry can be acquired by $\mathrm{CT}$ scan, and the material properties, especially nonlinear recovery pattern, should be evaluated by in vitro mechanical tests of the passive tissues. In addition, the boundary conditions in the FE loading steps may need to be 
re-defined to represent the 3D movement of the lumbar spine and the influence of the upper and lower extremities. 


\section{CONCLUSIONS}

Prolonged stooped posture in industry has been related to low back pain or disorders but no known tool to assess its risks has been developed. This study examined the viscoelastic behaviors in the in vivo lumbar spine during stooping and recovery period in terms of the range of full lumbar flexion in sagittal plane and the myoelectric activity of back extensor muscles in isokinetic lifting tasks with and without a short upright-standing rest break in the middle of stooping period. Results of this experimental study were used to validate a finite element (FE) model, which was developed simultaneously to predict mechanical responses in the lumbar spine in stooping and recovery.

The in vivo experiment found that the full lumbar flexion angle and the EMG in lifting increased in stooping and decreased in upright standing recovery period, indicating degraded lumbar spine stability and greater demand on the contribution of muscles to sustaining the stability and supporting flexion moment. These results suggested that tasks that require substantial amount of muscle exertion after prolonged stooping, such as lifting a filled bucket after picking up crops in agriculture field, could result in acute injury or disorder in the low back. Results indicated that the risks of prolonged stooping could be moderated by taking a short rest break in the middle of stooping.

The FE model of the lumbar spine was developed with nonlinear viscoelastic material properties for passive tissues. The model simulated the same posture and load as the in vivo experiment and the predicted results were compared with the experimental observations. The correlation coefficients between the FE model results and in vivo observations were greater than 0.97 and 0.91 for the full flexion angle and muscle force (EMG) variables, respectively. The validation processes showed good agreement in terms of time-dependent mechanical 
behaviors in stooping, confirming the capability of the FE model as a potential tool for risk assessment of the prolonged stooping tasks in industry. 


\section{REFERENCES}

Adams, M.A., Hutton, W.C., and Stott, J.R.R. (1980) The resistance to flexion of the lumbar intervertebral joint. Spine, 5 (3): 245-253.

Adams, M.A., Dolan, P., and Hutton, W.C. (1987) Diurnal-variations in the stresses on the lumbar spine. Spine, 12 (2): 130-137.

Adams, M.A., and Dolan, P. (1996) Time-dependent changes in the lumbar spine's resistance to bending. Clinical Biomechanics, 11 (4): 194-200.

Andersson, G.B.J. (1998) Epidemiology of low back pain, Acta Orthopaedica Scandinavica Suppl, 69 (Suppl.281): 28-31.

Avela, J., Finni, T., Liikavainio, T., Niemelä, E., and Komi, P.V. (2004) Neural and mechanical responses of the triceps surae muscle group after $1 \mathrm{~h}$ of repeated fast passive stretches. Journal of Applied Physiology, 96 (6): 2325-2332.

Ayoub, M.M., Selan,.L., and Liles, D.H. (1983) An ergonomics approach for the design of manual material-handling tasks. Human Factors, 25 (5): 507-515.

Bayraktar, H.H., Morgan, E.F., Niebur, G.L., Morris, G.E., Wong, E.K., and Keaveny, T.M. (2004) Comparison of the elastic and yield properties of human femoral trabecular and cortical bone tissue. Journal of Biomechanics, 37 (1): 27-35.

Belytschko, T., Kulak, R.F., Schultz, A.B., Galante, J.O. (1974) Finite element stress analysis of an intervertebral disc. Journal of Biomechanics, 7 (3): 277-285.

Best, B.A., Guilak, F., Setton, L.A., Zhu, W.B., Saed-Nejad, F., Ratcliffe, A., Weidenbaum, M., and Mow, V.C. (1994) Compressive mechanical properties of the human annulus fibrosus and their relationship to biochemical composition. Spine, 19 (2): 212-221.

Bobbert, M.F., Ettema, G.C., and Huijing, P.A. (1990) The force-length relationship of a muscle-tendon complex: experimental results and model calculations. European Journal of Applied Physiology, 61(3-4): 323-329.

Bogduk, N., Pearcy, M., and Hadfield, G. (1992) Anatomy and biomechanics of psoas major. Clincial Biomechanics, 7 (2): 109-119.

Bogduk, N., Macintosh, J.E., and Pearcy, M.J. (1992) A universal model of the lumbar back muscles in the upright position. Spine, 17 (8): 897-913.

Bogduk, N., Johnson, G., and Spalding, D. (1998) The morphology and biomechanics of latissimus dorsi. Clinical Biomechanics, 13 (6): 377-385. 
Bohannon, R.W., Gajdosik, R.L., and Le Veau, B.F. (1985a) Relationship of pelvic and thigh motions during unilateral and bilateral hip flexion. Physical Therapy, 65 (10): 1501-1504.

Bohannon, R.W., Gajdosik, R.L., and Le Veau, B.F. (1985b) Contribution of pelvic and lower limb motion to increases in the angle of passive straight leg raising. Physical Therapy, 65 (4): 474-476.

Bonfield, W., and Grynpas, M.D. (1977) Anisotropy of the young's modulus of bone. Nature, 270 (5636): 453-454.

Breau, C., Shirazi-Adl, A., and De Guise, J. (1991) Reconstruction of a human ligamentous lumbar spine using CT images - A three-dimensional finite element mesh generation. Annals of Biomedical Engineering, 19 (3): 291-302.

Bureau of labor statistics report (2004), http://www.bls.gov/iif/oshwc/osh/case/ostb1272.txt

Burns, M.L., Kaleps, I., and Kazarian, L.E. (1984) Analysis of compressive creep behavior of the vertebral unit subjected to uniform axial loading using exact parametric solution equation of Kelvin-solid models - Part 1. Human intervertebral joints. Journal of Biomechanics, 17 (2): 113-130.

Burstein, A.H., Reilly, D.T., and Martens, M. (1976) Aging of bone tissue: mechanical properties. The Journal of Bone and Joint Surgery, 58 (1): 82-86.

Chaffin, D.B., Andersson, G.B.J., and Martin, B.J. (1999) Occupational biomechanics $\left(^{\text {rd }}\right.$ Ed.). New York, NY: John Wiley \& Sons.

Chazal, J., Tanguy, A., Bourges, M., Gaurel, G., Escande, G., Guillot, M., and Vanneuville, G. (1985) Biomechanical properties of spinal ligaments and a histological study of the supraspinal ligament in traction. Journal of Biomechanics, 18 (3): 167-176.

Cheung, J.T., Zhang, M., and Chow, D.H. (2003) Biomechanical responses of the intervertebral joints to static and vibrational loading: a finite element study. Clinical Biomechanics, 18 (9): 790-799.

Cholewicki, J., and McGill, S.M. (1992) Lumbar posterior ligament involvement during extremely heavy lifts estimated from fluoroscopic measurements. Journal of Biomechanics, 25 (1): 17-28.

Cholewicki, J., and McGill, S.M. (1996) Mechanical stability of the in vivo lumbar spine: implications for injury and chronic low back pain. Clinical Biomechanics, 11 (1): 1-15.

Crisco, J.J., Panjabi, M.M., Yamamoto, I., and Oxland, T.R. (1992) Euler stability of the human ligamentous lumbar spine. Part II: Experiment. Clinical Biomechanics, 7 (1): $27-$ 32. 
Dickey, J.P., McNorton, S., and Potvin, J.R. (2003) Repeated spinal flexion modulates the flexion-relaxation phenomenon. Clinical Biomechanics, 18 (9): 783-789.

Dolan, P., and Adams, M.A. (1993) Influence of lumbar and hip mobility on the bending stresses acting on the lumbar spine. Clinical Biomechanics, 8 (4): 185-192.

Dolan, P., Mannion, A.F., and Adams, M.A. (1994) Passive tissues help the back muscles to generate extensor moments during lifting. Journal of Biomechanics, 27 (8): 1077-1085.

Dumas, G.A., Beaudoin, L., and Drouin, G. (1987) In situ mechanical behavior of posterior spinal ligaments in the lumbar region. An in vitro study. Journal of Biomechanics, 20 (3): 301-310.

Esola, M.A., McClure, P.W., Fitzgerald, G.K., and Siegler, S. (1996) Analysis of lumbar spine and hip motion during forward bending in subjects with and without a history of low back pain. Spine, 21 (1): 71-78.

Evans, F.G. (1972) Mechanical properties of bone. Charles C. Thomas. Springfield, Illinoise.

Eversull, E., Solomonow, M., Zhou, B.H, Baratta, R.V., and Zhu, M.P. (2001) Neuromuscular neutral zones sensitivity to lumbar displacement rate. Clinical Biomechanics, 16 (2): 102-113.

Floyd, W.F., and Silver, P.H.S. (1955) The function of the erectors spinae muscles in certain movements and postures in man. Journal of Physiology, 129 (1): 184-203.

Fowles, J.R., Sale, D.G., and MacDougall, J.D. (2000) Reduced strength after passive stretch of human plantarflexors. Journal of Applied Physiology, 89 (3): 1179-1188.

Fung, Y.C. (1993) Biomechanics: mechanical properties of living tissues $\left(2^{\text {nd }}\right.$ Ed.). New York: Springer-Verlag.

Galante, J.O. (1967) Tensile properties of the human lumbar annulus fibrosus. Acta Orthopaedica Scandinavica, S100 : 1-91.

Galante, J., Rostoker, W., and Ray, R.D. (1970) Physical properties of trabecular bone. Calsified Tissue Research, 5 (3): 236-246.

Gareis, H., Solomonow, M., Baratta, R., Best, R., and D’Ambrosia, R. (1992) The isometric length-force models of nine different skeletal muscles. Journal of Biomechanics, 25 (8): 903-916.

Goel, V.K., and Kim, Y.E. (1989) Effects of injury on the spinal motion segment mechanics in the axial compression mode. Clinical Biomechanics, 4 (3): 161-167. 
Goel, V.K., Kong, W.Z., Han, J.S., Weinstein, J.N., and Gilbertson, L.G. (1993) A combined finite element and optimization investigation of lumbar spine mechanics with and without muscles. Spine, 18 (11): 1531-1541.

Goel, V.K., Monroe, B.T., Gilbertson, L.G., Brinckmann, P., and Nat, R. (1995) Interlaminar shear stresses and laminae separation in a disc - Finite element analysis of the L3-L4 motion segment subjected to axial compressive loads. Spine, 20 (6): 689-698.

Gracovetsky, S., Farfan, H., and Lamy, C. (1981) The mechanism of the lumbar spine. Spine, 6 (3): 249-262.

Gracovetsky, S., Newman, N., Pawlowsky, M., Lanzo, V., Davey, B., and Robinson, L. (1995) A database for estimating normal spinal motion derived from noninvasive measurements. Spine, 20 (9): 1036-1046.

Guo, H., Tanaka, S., Halperin, W.E., and Cameron, L.L. (1999) Back pain prevalence in US industry and estimates of lost workdays. American Journal of Public Health, 89 (7): 1029-1035.

Gupta, A. (2001) Analyses of myo-electrical silence of erectors spinae. Journal of Biomechanics, 34 (4): 491-496.

Hawkins, D., and Bey, M. (1997) Muscle and tendon force-length properties and their interactions in vivo. Journal of Biomechanics, 30 (1): 63-70.

Hayes, M.A., Howard, T.C., Gruel, C.R., and Kopta, J.A. (1989) Roentgenographic evaluation of lumbar spine flexion-extension in asymptomatic individuals. Spine, 14 (3): 327-331.

Iatridis, J.C., Weidenbaum, M., Setton, L.A., and Mow, V.C. (1996) Is the nucleus pulposus a solid or a fluid? Mechanical behaviors of the nucleus pulposus of the human intervertebral disc. Spine, 21 (10): 1174-1184.

Keller, T.S., Holm, S.H., Hansson, T.H. and Spengler, D.M. (1990) The dependence of intervertebral disc mechanics properties on physiologic conditions. Spine, 15 (8): 751761.

Kippers, V., and Parker, A.W. (1984) Posture related to myoelectric silence of erectors spinae during trunk flexion. Spine, 9 (7): 740-745.

Kraus, J.F., Schaffer, K.B., McArthur, D.L., and Peek-Asa, C. (1997) Epidemiology of acute low back pain injury in employees of a large home improvement retail company. Americal Journal of Epidemiology, 146 (8): 637-645. 
Kubo, K., Kanehisa, H., and Fukunaga, T. (2002) Effects of transient muscle contractions and stretching on the tendon structures in vivo. Acta Physiologica Scandinavica, 175 (2): 157-164.

Kulak, R.F., Belytschko, T.B., Schultz, A.B., and Galante, J.O. (1976) Nonlinear behavior of the human intervertebral disc under axial load. Journal of Biomechanics, 9 (6): 377-386.

Li, S.P., Patwardhan, A.G., Amirouche, F.M.L., Havey, R., and Meade, K.P. (1995) Limitations of the standard linear solid model of intervertebral discs subject to prolonged loading and low-frequency vibration in axial compression. Journal of Biomechanics, 28 (7): 779-790.

Liu, Y.K., Ray, G., and Hirsch, C. (1975) The resistance of the human spine to direct shear. Orthopedic Clinics of North America, 6 (1): 33-48.

Lu, Y.M., Hutton, W.C., and Gharpuray, V.M. (1998) The effect of fluid loss on the viscoelastic behavior of the lumbar intervertebral disc in compression. Journal of Biomechanical Engineering, 120 (1): 48-54.

Macintosh, J.E., and Bogduk, N. (1987) The morphology of the lumbar erector spinae. Spine, 12 (7): 658-668.

Magnusson, S.P. (1998) Passive properties of human skeletal muscle during stretch maneuvers. Sandinavian Journal of Medicine \& Science in Sports, 8 (2): 65-77.

Magnusson, S.P., Aagaard, P., and Nielson, J.J. (2000) Passive energy return after repeated stretches of the hamstring muscle-tendon unit. Medicine and Science in Sports and Exercise, 32 (6): 1160-1164.

Marchand, F., and Ahmed, A.M. (1990) Investigation of the laminate structure of lumbar disc annulus fibrosus. Spine, 15 (5): 402-410.

Markolf, K.L., and Morris, J.M. (1974) The structural components of the intervertebral disc. Journal of Bone and Joint Surgery, 56 (4): 675-687.

Marras, W.S., Lavender, S.A., Leurgans, S.E., Fathallah, F.A., Ferguson, S.A., Allread, W.G., and Rajulu, S.L. (1995) Biomechanical risk factors for occupationally related low back disorders. Ergonomics, 38 (2): 377-410.

McGill, S.M., and Brown, S. (1992) Creep responses of the lumbar spine to prolonged full flexion. Clinical Biomechanics, 7 (1): 43-46.

McGill, S.M., and Kippers, V. (1994) Transfer of loads between lumbar tissues during the flexion-relaxation phenomenon. Spine, 19 (19): 2190-2196. 
McGill, S.M. (1996) A revised anatomical model of the abdominal musculature for torso flexion efforts. Journal of Biomechanics, 29 (7): 973-977.

McGill, S.M. (1997) The biomechanics of low back injury: Implications on current practice in industry and the clinic. Journal of Biomechanics, 30 (5): 465-475.

McGill, S. (2002) Low back disorders. Evidence-based prevention and rehabilitation. Human Kinetics.

McHugh, M.P., Magnusson, S.P., Gleim, G.W., and Nicholas, J.A. (1992) Viscoelastic stress relaxation in human skeletal muscle. Medicine and Science in Sports and Exercise, 24 (12): $1375-1382$.

McNally, D.S., and Adams, M.A. (1992) Internal intervertebral disc mechanics as revealed by stress profilometry. Spine, 17 (1): 66-73.

Montgomery, D.C. (2001) Design and analysis of experiments (5 ${ }^{\text {th }}$ Ed.). New York: John Wiley \& Sons.

Mosekilde, L., Moseklde, L., and Danielsen, C.C. (1987) Biomechanical competence of vertebral trabecular bone in relation to ash density and age in normal individuals. Bone, 8 (2): 79-85.

Murray, R., Bohannon, R.W., Tiberio, D., Dewberry, M.J., and Zannotti, C. (2002) Pelvifemoral rhythm during unilateral hip flexion in standing. Clinical Biomechanics, 17 (2): $147-151$.

Myklebust, J.B, Pintar, F., Yoganandan, N., Cusick, J.F., Maiman, D., Myers, T.J., and Sances, A. (1988) Tensile strength of spinal ligaments. Spine, 13(5): 526-531.

Nachemson, A.L, and Evans, J.H. (1968) Some mechanical properties of the third human lumbar interlaminar ligament (Ligamentum flavum). Journal of Biomechanics, 1: 211220 .

Nachemson, A.L. (1985) Advances in low-back pain. Clinical Orthopaedics and Related Research, 200: 266-278.

Neumann, P., Keller, T.S., Ekström, L., Perry, L., Hansson, T.H., and Spengler, D.M. (1992) Mechanical properties of the human lumbar anterior longitudinal ligament. Journal of Biomechanics, 25 (10): 1185-1194.

Panjabi, M.M., Goel, V.K., and Takata, K. (1982) Physiologic strains in the lumbar spinal ligaments. Spine, 7 (3): 192-203. 
Panjabi, M.M., Goel, V., Oxland, T., Takata, K., Duranceau, J., Krag, M., and Price, M. (1992) Human lumbar vertebrae. Quantitative three-dimensional anatomy. Spine, 17 (3): 299-306.

Pearcy, M., Portek, I., and Shepherd, J. (1984) Three-dimensional X-ray analysis of normal movement in the lumbar spine. Spine, 9 (3): 294-297.

Pearcy, M.J., and Tibrewal, S.B. (1984) Lumbar intervertebral disc and ligament deformations measured in vivo. Clinical Orthopaedics and Related Research, 191: 281286.

Pintar, F.A., Yoganandan, N., Myers, T., Elhagediab, A., and Sances, A. (1992) Biomechanical properties of human lumbar spine ligaments. Journal of Biomechanics, 25 (11): 1351-1356.

Pope, M.H., and Panjabi, M.M. (1985) Biomechanical definitions of spinal instability. Spine, 10 (3): $255-256$.

Pope, M.H. (1989) Risk indicators in low back pain. Annals of Medicine, 21 (5): 387-392.

Punnett, L., Fine, L.J., Keyserling, W.M., Herrin, G.D., and Chaffin, D.B. (1991) Back disorders and non-neutral trunk postures of automobile assembly workers. Scandinavian Journal of Work Environment and Health, 17 (5): 337-346.

Reid, J.G., and Costigan, P.A. (1987) Trunk muscles balance and muscular force. Spine, 12 (8): 783-786.

Sarver, J.J., Robinson, P.S., and Elliott, D.M. (2003) Methods for quasi-linear viscoelastic modeling of soft tissue: Application to incremental stress-relaxation experiments. Journal of Biomechanical Engineering, 125 (5): 754-758.

Schultz, A.B., Haderspeck-Grib, K., Sinkora, G., and Warwick, D.N. (1985) Quantitative studies of the flexion-relaxation phenomenon in the back muscles. Journal of Orthopaedic Research, 3 (2): 189-197.

Sharma, M., Langrana, N.A., and Rodriguez, J. (1995) Role of ligaments and facets in lumbar spinal stability. Spine, 20 (8): 887-900.

Shin, G., Shu, Y., Li, Z., Jiang, Z., and Mirka, G. (2004) Influence of knee angle and individual flexibility on the flexion-relaxation response of the low back musculature. Journal of Electromyography and Kinesiology, 14 (4): 485-494.

Shirazi-Adl, S.A., Shrivastava, S.C., and Ahmed, A.M. (1984) Stress analysis of the lumbar disc-body unit in compression. A three-dimensional nonlinear finite element study. Spine, 9 (2): 120-134. 
Shirazi-Adl, A., Ahmed, A.M., and Shrivastava, S.C. (1986) A finite element study of a lumbar motion segment subjected to pure sagittal plane moments. Journal of Biomechanics, 19 (4): 331-350.

Shirazi-Adl, A., and Parnianpour, M. (1993) Nonlinear response analysis of the human ligamentous lumbar spine in compression. Spine, 18 (1): 147-158.

Shirazi-Adl, A. (1994) Biomechanics of the lumbar spine in sagittal/lateral moments. Spine, 19 (21): 2407-2414.

Shirazi-Adl, A., Sadouk, S., Parnianpour, M., Pop. D., and El-Rich, M. (2002) Muscle force evaluation and the role of posture in human lumbar spine under compression. European Spine Journal, 11 (6): 519-526.

Simoneau, G.G. (1998) The impact of various anthropometric and flexibility measurements on the sit-and-reach test. Journal of Strength and Conditioning Research, 12 (4): 232-237.

Skaggs, D.L., Weidenbaum, M., Iatridis, J.C., Ratcliffe, A., and Mow, V.C. (1994) Regional variation in tensile properties and biomechanical composition of the human lumbar annulus fibrosus. Spine, 19 (12): 1310-1319.

Solomonow, M., Zhou, B.H., Baratta, R.V., Lu, Y., Zhu, M.P., and Harris, M. (2000) Biexponential recovery model of lumbar viscoelastic laxity and reflexive muscular activity after prolonged cyclic loading. Clinical Biomechanics, 15 (3): 167-175.

Solomonow, M., Baratta, R.V., Banks, A., Freudenberger, C., and Zhou, B.H. (2003a) Flexion-relaxation response to static lumbar flexion in males and females. Clinical Biomechanics, 18 (4): 273-279.

Solomonow, M., Baratta, R.V., Zhou, B.H., Burger, E., Zieske, A., and Gedalia, A. (2003b) Muscular dysfunction elicited by creep of lumbar viscoelastic tissue. Journal of Electromyography and Kinesiology, 13 (4): 381-396.

Solomonow, M. (2004) Ligaments: a source of work-related musculoskeletal disorders. Journal of Electromyography and Kinesiology, 14 (1): 49-60.

Spilker, R.L. (1980) Mechanical behavior of a simple model of an intervertebral disk under compressive loading. Journal of Biomechanics, 13 (10): 895-901.

Stokes, I.A.F., and Gardner-Morse, M. (1999) Quantitative anatomy of the lumbar musculature. Journal of Biomechanics, 32 (3): 311-316.

Takashima, S.T., Singh, S.P., Haderspeck, K.A., and Schultz, A.B. (1979) A model for semiquantitative studies of muscle actions. Journal of Biomechanics, 12 (12): 929-939. 
Taylor, D.C., Dalton, J.D., Seaber, A.V., and Garrett, W.E. (1990) Viscoelastic properties of muscle-tendon units. The biomechanical effects of stretching. The American Journal of Sports Medicine, 18 (3): 300-309.

Taylor, D.C., Brooks, D.E., and Ryan, J.B. (1997) Viscoelastic characteristics of muscle: passive stretching versus muscular contractions. Medicine and Science in Sports and Exercise, 29 (12): 1619-1624.

Van Dieën, J.H., Toussaint, H.M., Stam, C., and Hol, J. (1994) Viscoelasticity of the individual spine. Clinical Biomechanics, 9 (1): 61-63.

Von Korff, M., Dworkin, S.F., Le Resche, L., and Kruger, A. (1988) An epidemiologic comparison of pain complaints. Pain, 32 (2): 173-183.

Wang, J.L., Parnianpour, M., Shirazi-Adl, A., Engin, A.E., Li, S., and Patwardhan, A. (1997) Development and validation of a viscoelastic finite element model of an L2/L3 motion segment. Theoretical and Applied Fracture Mechanics, 28 (1): 81-93.

Wang, J.L., Parnianpour, M., Shirazi-Adl, A., and Engin, A.E. (1999) Rate effect on sharing of passive lumbar motion segment under load-controlled sagittal flexion: viscoelastic finite element analysis. Theoretical and Applied Fracture Mechanics, 32 (2): 119-128.

Wang, J.L., Parnianpour, M., Shirazi-Adl, A., and Engin, A.E. (2000) Viscoelastic finiteelement analysis of a lumbar motion segment in combined compression and sagittal flexion. Spine, 25 (3): 310-318.

Waters, T.R., Baron, S.L., Piacitelli, L.A., Anderson, V.P., Skov, T., Haring-Sweeney, M., Wall, D.K., and Fine, L.J. (1999) Evaluation of the revised NIOSH lifting equation. A cross-sectional epidemiology study. Spine, 24 (4): 386-394.

Webster, B.S., and Snook, S.H. (1994) The cost of 1989 workers' compensation low back pain claims. Spine, 19 (10): 1111-1116.

White, A.A, and Panjabi, M.M. (1990) Clinical biomechanics of the spine (2 ${ }^{\text {nd }}$ Ed.). Philadelphia: Lippincott Williams \& Wilkins.

Winter, D. (2005) Biomechanics and motor control of human movement ( $3^{\text {rd }}$ Ed.). Hobboken, NJ: John Wiley \& Sons.

Xu, Y., Bach, E., and Ørhede, E (1997) Work environment and low back pain: the influence of occupational activities. Occupational and Environmental Medicine, 54 (10): 741-745.

Yahia, L.H., Audet, J., and Drouin, G. (1991) Rheological properties of the human lumbar spine ligaments. Journal of Biomedical Engineering, 13 (5): 399-406. 
Yamada, H., and Evans, F.G. (1970) Strength of biological materials. Baltimore: The Williams \& Wilkins Company.

Yamamoto, I., Panjabi, M.M., Oxland, T.R., and Crisco, J.J. (1989) Three-dimensional movements of the whole lumbar spine and lumbosacral joint. Spine, 14 (11): 1256-1260.

Zander, T., Rohlmann, A., Calisse, J., and Bergmann, G. (2001) Estimation of muscle forces in the lumbar spine during upper-body inclination. Clinical Biomechanics, 16: S73-80.

Zander, T., Rohlmann, A., and Bergmann, G. (2004) Influence of ligament stiffness on the mechanical behavior of a functional spinal unit. Journal of Biomechanics, 37 (7): 11071111. 


\section{APPENDIX : Adequacy of the Statistical Model}

The normality of residuals and the equality of variance from the statistical models were checked graphically to validate the adequacy of the MANOVA and ANOVA models (Montgomery, 2001). The normality assumption was tested by examining normal probability

plots of residuals (difference between observed value and fitted value). The equality of variance assumption was tested by plotting the residuals versus the fitted values.

Normal probability plots of residuals of all dependent variables showed no severe indication of departure from normality and no possible outliers, and no apparent relationship between the residuals and the fitted values was found in the plots of the residuals versus fitted values. These examinations suggest that the assumptions are valid and the ANOVA models are adequate.

Following figures show the normal probability plots and the plots of residuals versus fitted values. 


\section{Full lumbar flexion angle}

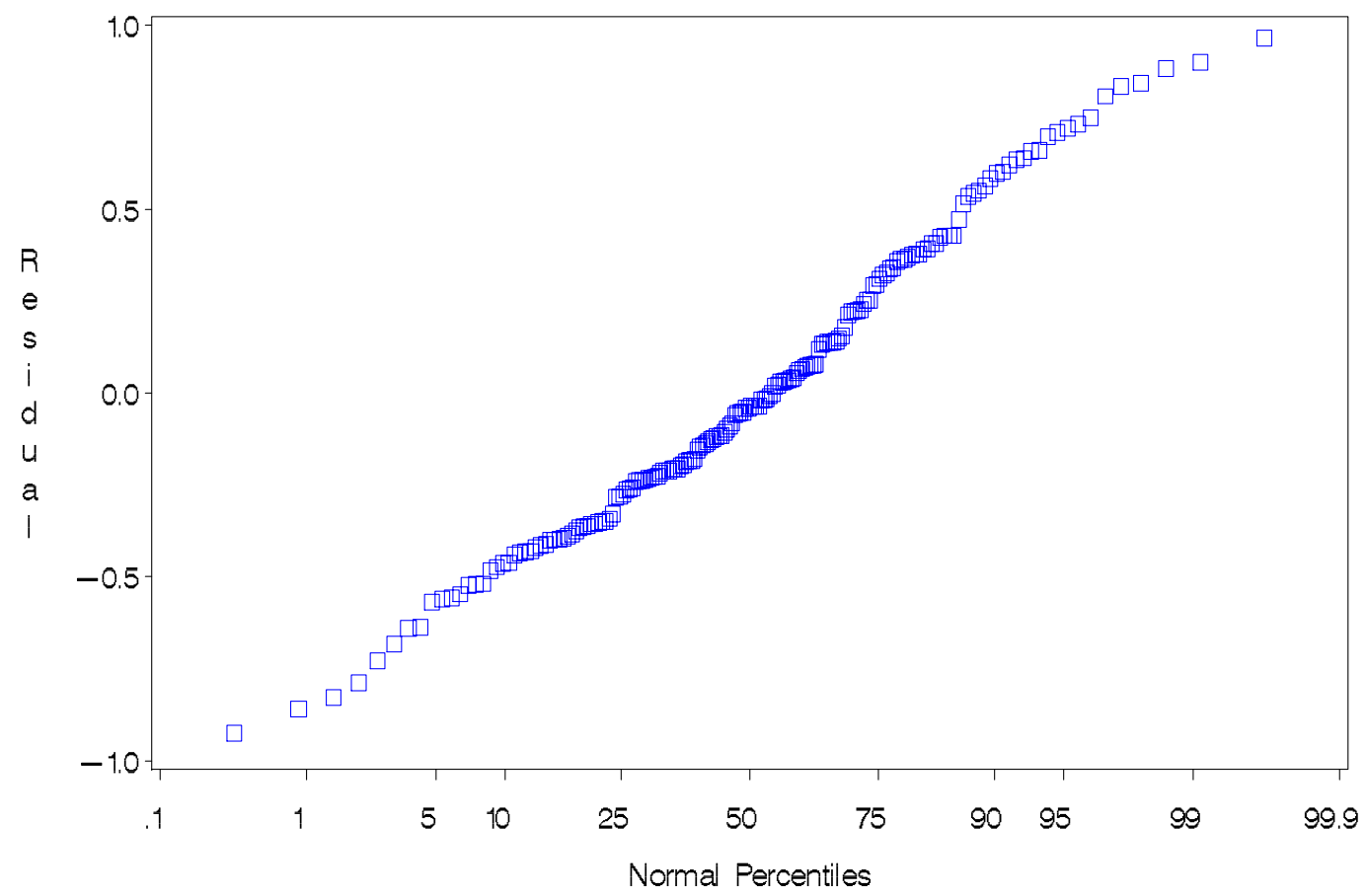

Normal probability of residuals

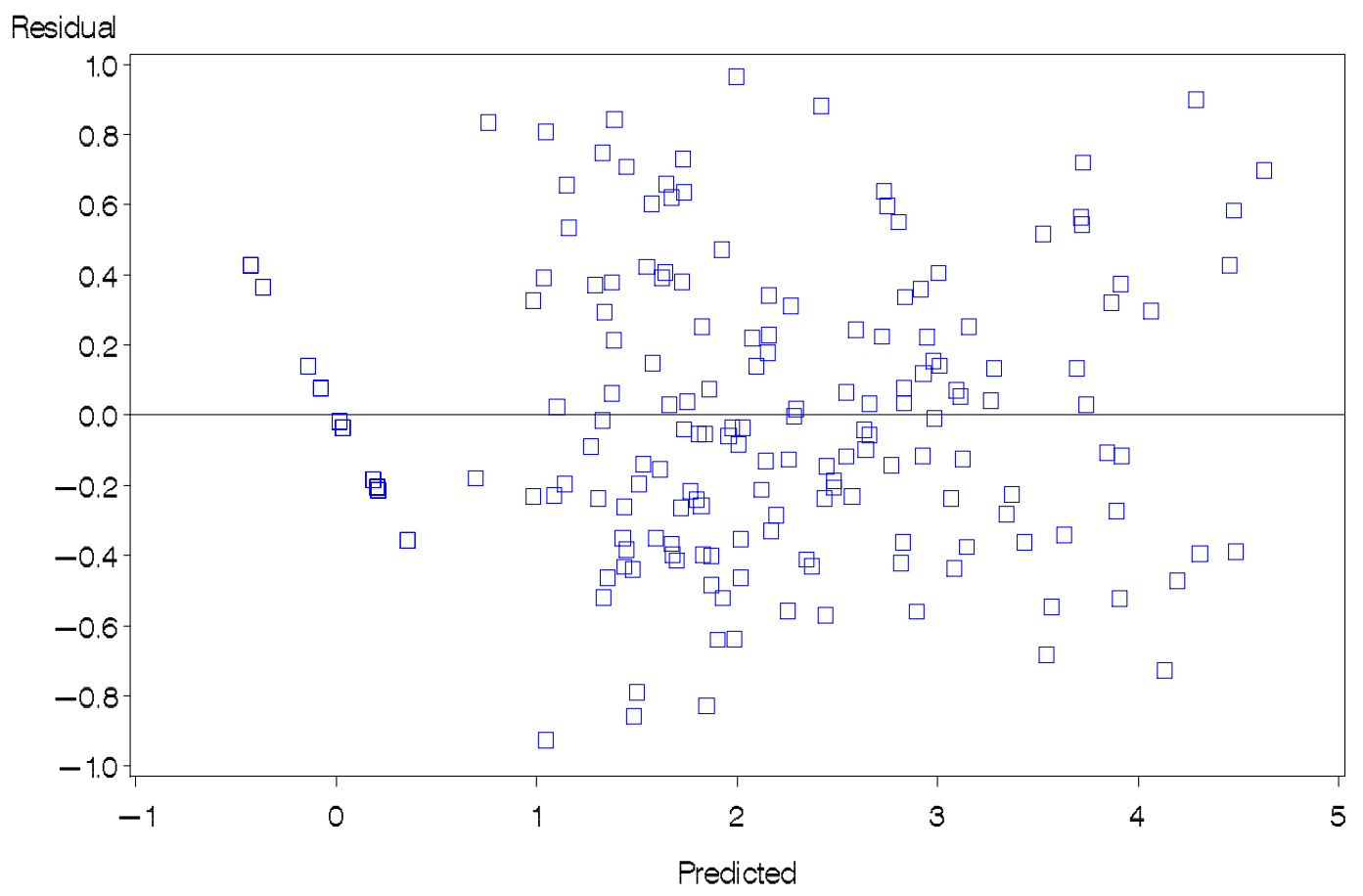

Plot of residuals versus fitted values 


\section{Mean EMG of multifidus}

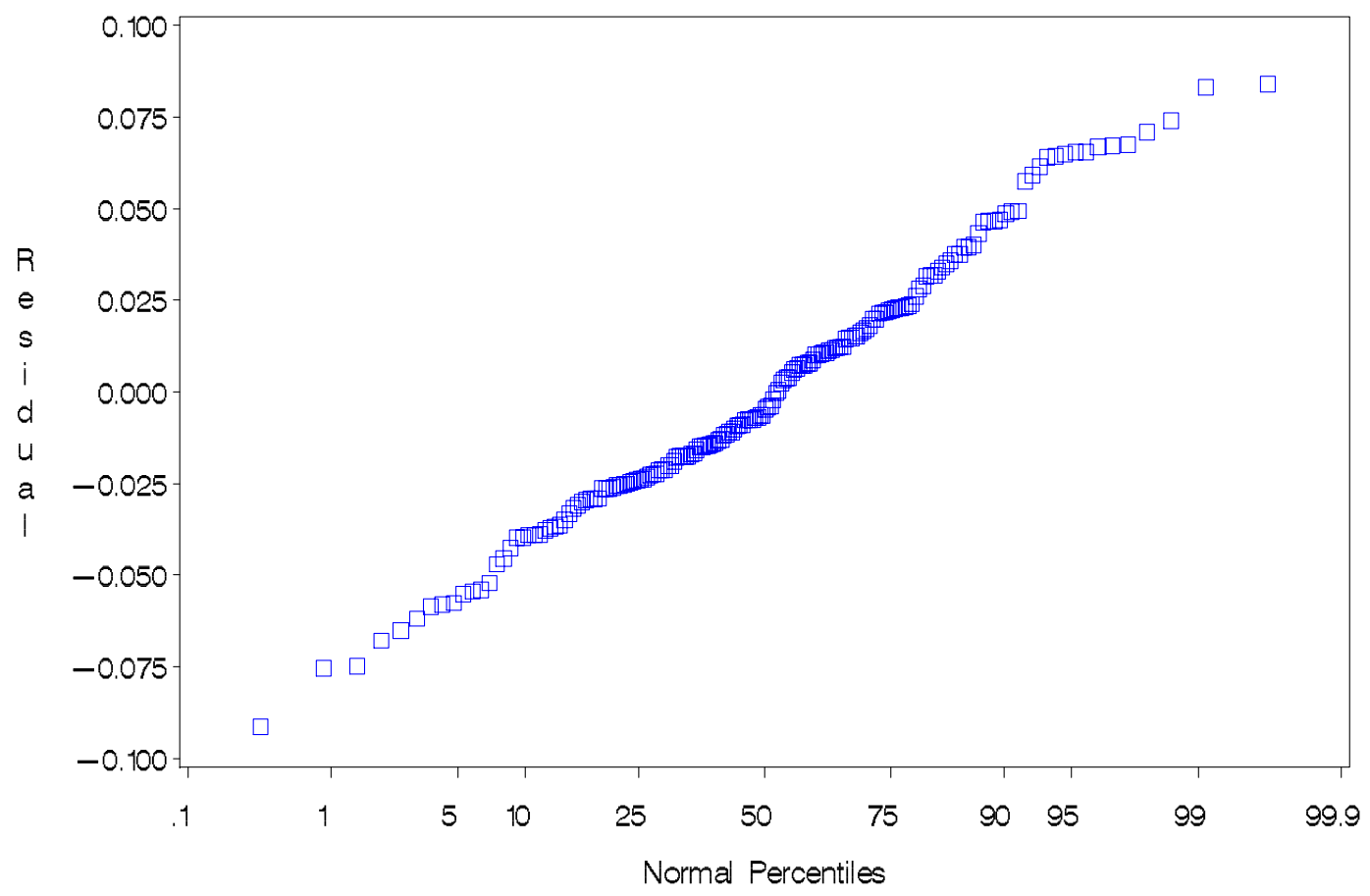

Normal probability of residuals

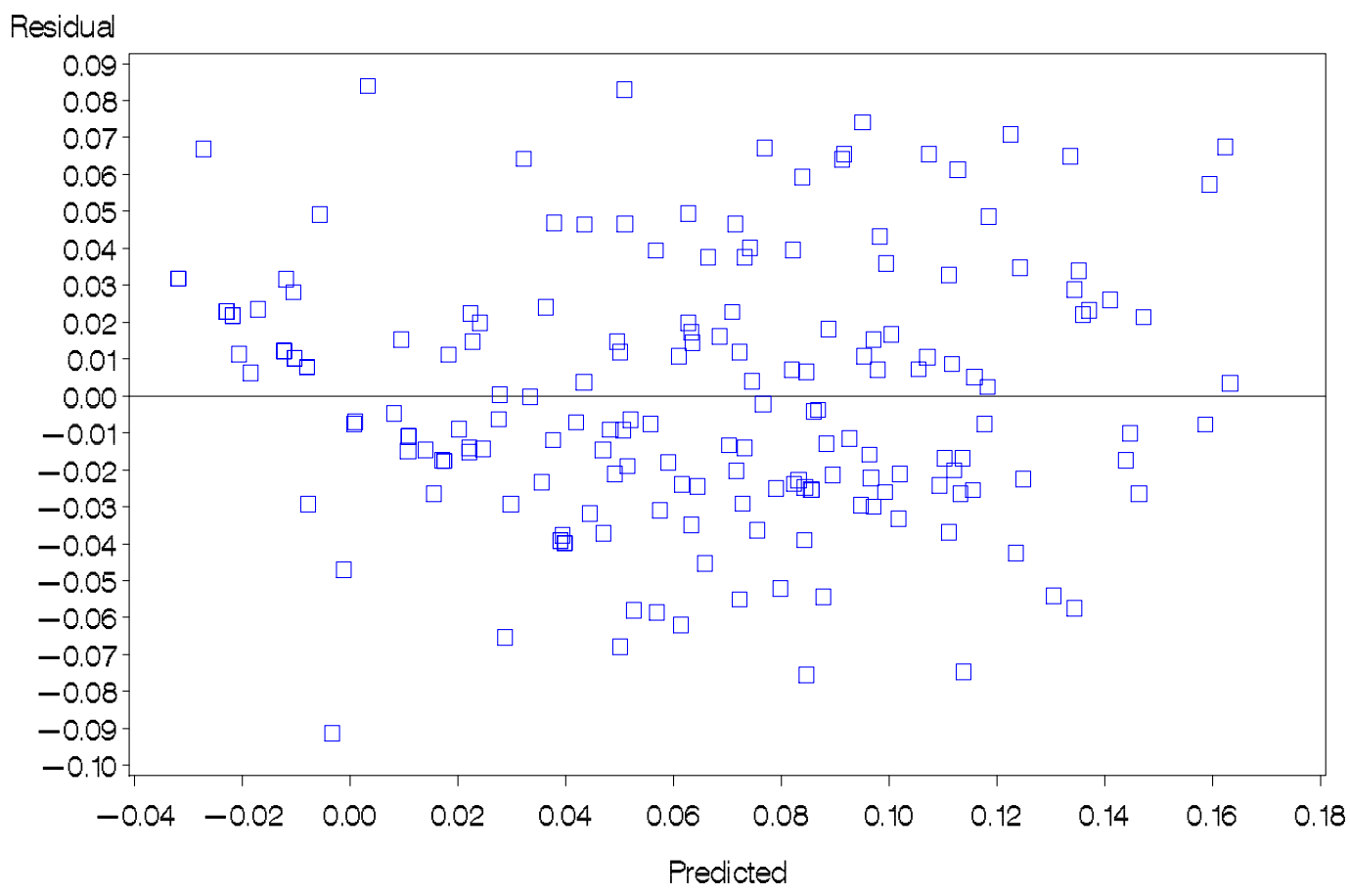

Plot of residuals versus fitted values 


\section{Mean EMG of erector spinae}

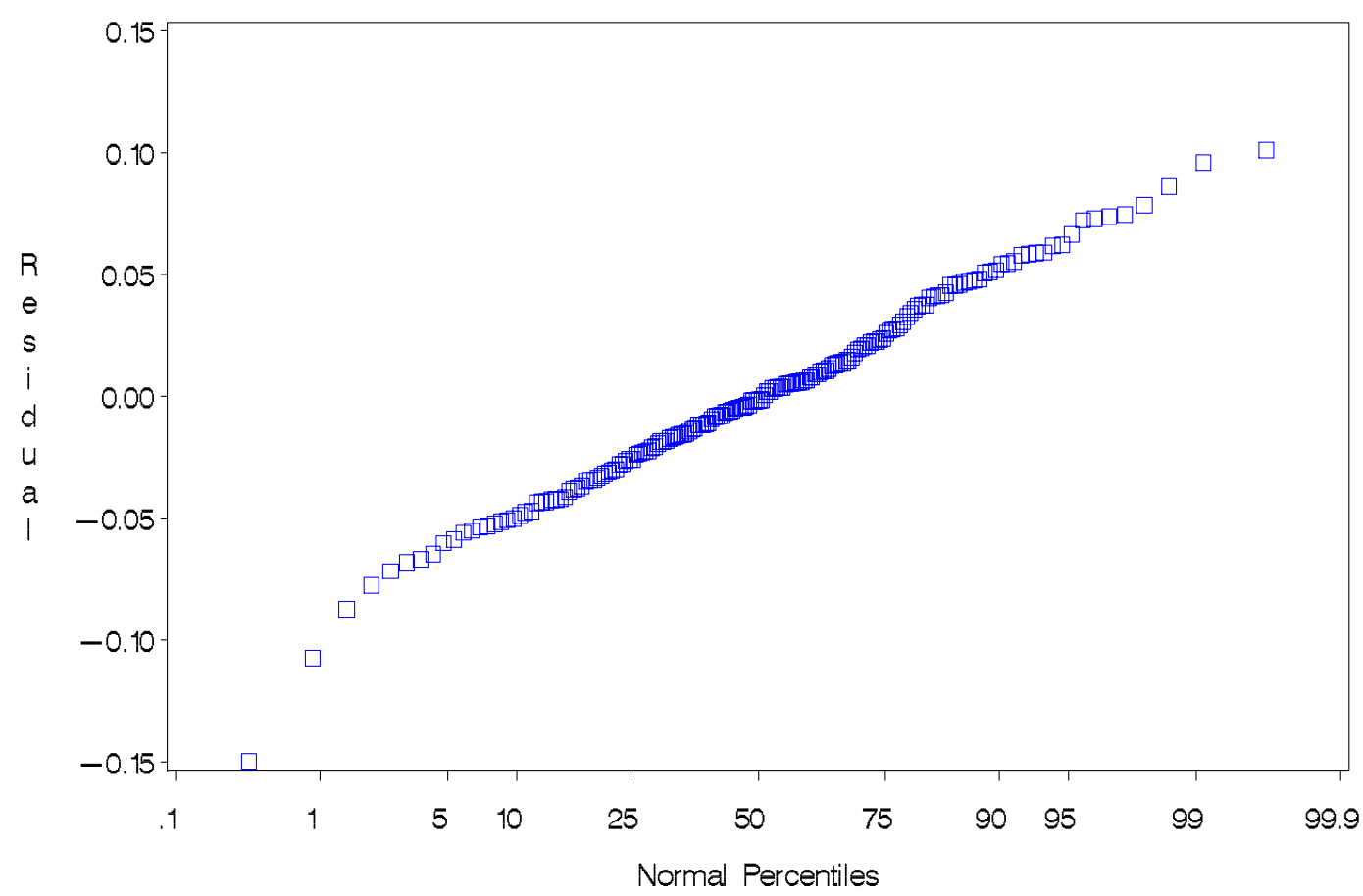

Normal probability of residuals

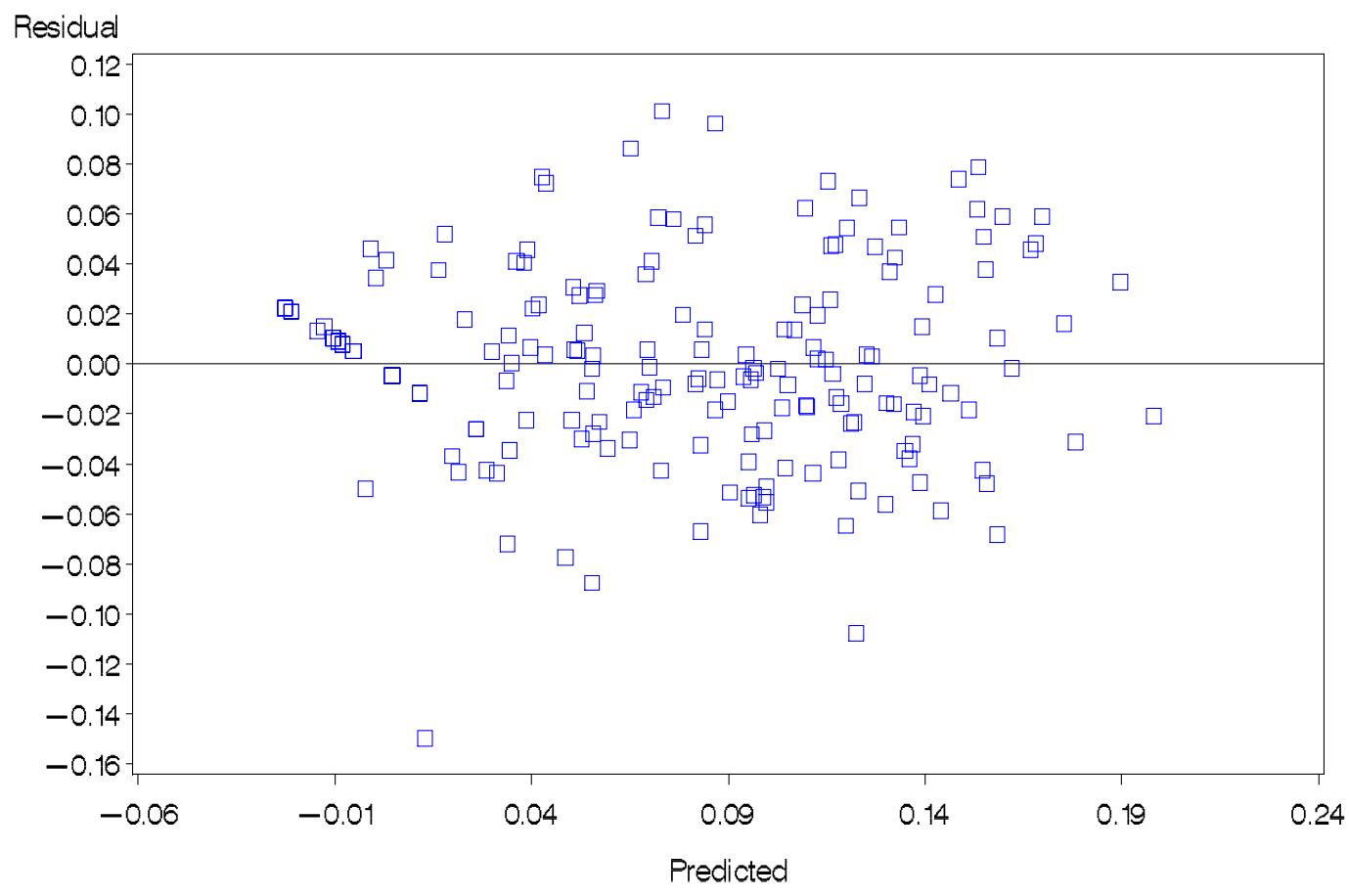

Plot of residuals versus fitted values 